\title{
THE FORMATION OF AMORPHOUS SILICON BY LIGHT ION DAMAGE
}

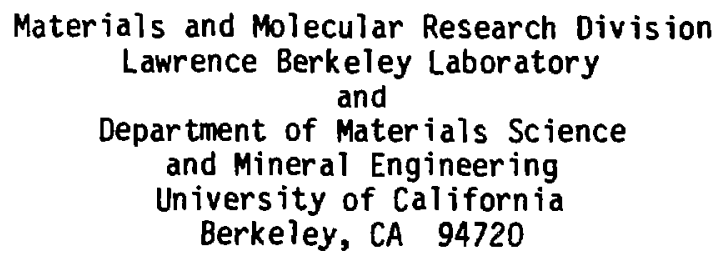

\section{MSTER}

\section{DISCLAMMER}

Thi report was prepared as an acoount of work sposeored by an aneacy of the Uaited States Governmeat. Neiluer the Unived Stales Gowernmeat wor any actacy therwof, nor way of their

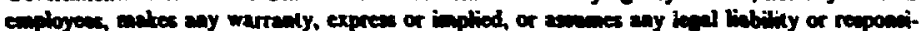

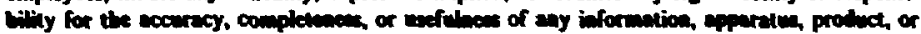

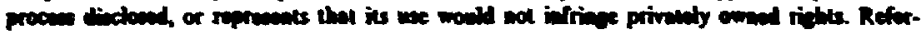

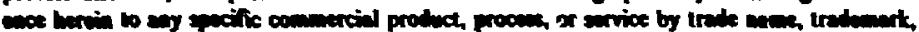

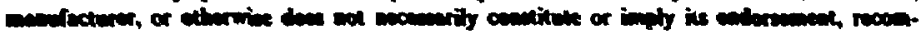

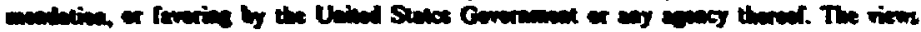

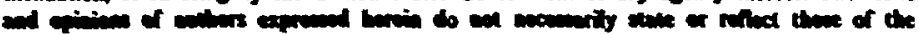

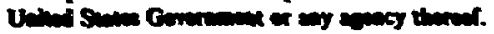

This work was supported by the Division of Materials science, office of Basic Energy Science, U.S. Departument of Energy, under contract wo. oc-ncos-76sfoodso. 


\section{yivi}

6.2 Amorphization Mechanism at Various

Temperatures and Ion Masses ......... 108

6.3 The Out-Diffusion of Silicon Interstitials

During Ion Implantation ............ 113

6.4 The Influence of High Beam Current Implantation

on Amorphization. ...................... 116

6.5 The Development of Secondary Defects

From Preliminary Defects............ 117

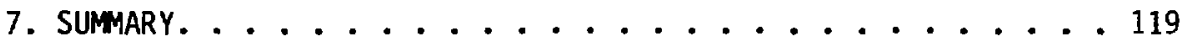

8. CONCLUSION . . . . . . . . . . . . . . 122

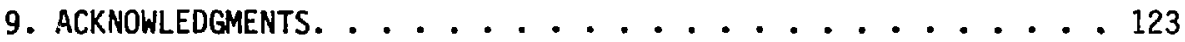

10. REFERENCES . . . . . . . . . . . . 125 
ABSTRACT ............................... vii

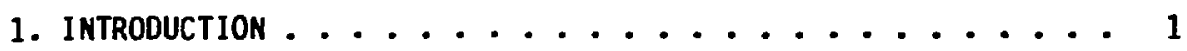

1.1 The Importance of a Continuous Amorphous Layer. . . . 2

1.2 Nuclear and Electronic Stopping ........... 5

1.3 Radiation Damage by Heavy Ion and Light Ion ...... 9

1.4 Heterogeneous and Homogeneous Nucleation. . . . . . 13

1.5 Two Stage Phenomenon in IR Reflection........ 14

1.6 The Present Work. . . . . . . . . . . 17

2. AMORPHOUS SILICON AND AMORPHIZATION. .......... 20

2.1 Amorphous Silicon ............. 20

2.1.1 The Structure of Amorphous Silicon .......20

2.1.2 Thermodynamics Criterion for the Crystalline-Amorphous Transition ...... 27

2.1.3 The First Order Phase Transition ........ 31

2.2 Amorphization Models. . . . . . . . . . 34

2.2.1 Heterogeneous Nucleation Models........ 34

2.2.2 Homogeneous Nucleation Models.......... 41

2.2.3 Critical Deposition Energy Criterion ...... 47

3. PROPOSED MHORPHIZATION MECHANISH FOR LIGHT ION DAMMGE. . . . 51

3.1 Two Possibie morphization Mechanisms ........ 51

3.2 A Model of Morphous Zone Formation by Light

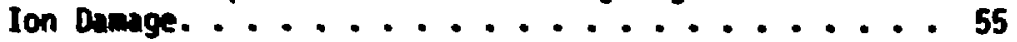

3.2.1 The Importance of Interstitial Type

Defects for Morphization. ........ 55

3.2.2 The Point Defect Clusterings ......... $57^{\circ}$

3.2.3 Embryo of an Morpmous Zone: The Palring of Divacenctes and Oilinterstitials...... 60 
4. EXPERIMENTAL BASIS FOR AMORPHIZATION MECHANISM . . . . . 67

4.1 Experimental Approach ............. 67

4.1.1 High Resolytion TEM Observation at AmorphousCrystalline Transition Regions in a CrossSection Specimen .......... 67

4.1.2 EPR Detection of Amorphous Zone Formation and Point Defect Clusters. . . . . . . 67

4.1.3 The Effects of Dose Rate (Beam Current) and Implantation Temperature on Amorphization. . . 69

4.1.4 High Voltage Ion Implantation. . . . . . . 69

4.2 Experimental Procedure............. 70

4.2.1 The Starting Materials and Ion Implantation. . . 70

4.2.2 Cross-Section Specimen Preparation for Transmission Electron Microscopy ...... 71

4.2.3 Lattice Imaging High Resolution Transmission

Electron Microscopy.......... 73

4.2.4 EPR (Electron Paramagnetic Resonance)

Measurement........... 75

5. EXPERIMENTAL RESULTS . . . . . . . . . . . . 79

5.1 The Discovery of Small Amorphous Zones. . . . . . 79

5.2 The Identification of Amorphous Silicon by EPR. . . . 81

5.3 The Formation of Stacking Faults. . . . . . . . 84

5.4 Effect of Predamage with Boron on the Formation of Amorphous Zones by Silicon .......... 96

5.5 The Formation of a Continuous Mmorphous Layer ...... 96

5.6 The Critical Deposition Energy for Rorphization. . . . 102

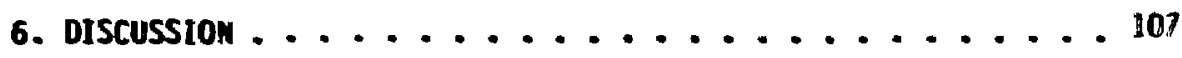

6.1 The Formation of Anorphous zones

by lignt ion ormage.............. 107 
THE FORMATION OF AMORPHOUS SILICON

BY LIGHT ION DAMAGE

Yih-Cheng Shih

Materials and Molecular Research Division

Lawrence Berkeley Laboratory

and

Department of Materials Science

and Mineral Engineering

University of California

Berkeley, CA 94720

\section{ABSTRACT}

Amorphization due to implantation of boron ions which is the lightest element used for I.C. fabrication processes, has been systematicaliy studied for various temperatures, voltages and dose rates. The formation of amorphous zones by boron ion implantation at room temperature is observed by cross-section high resolution TEM lattice images and by the electron paramagnetic resonance technique. Continuous amorphous material cannot be formed by boron ion implantation at room temperature, unless a high beam current is employed. (113), $1 / 3(111)$ extrinsic and other smaller distortion $1 / \times(111)$ stacking faults were found to be formed in the still-crystalline material during amorphization at roon temperature. These stacking faults are consioered as the origin of the secondary defects formed during annealing.

A model for formation of amorphous silicon by light ion implantation is proposeo. It is suggested that accumalation of point defects and/or clusters is required at the initial stage of amphization process. Amorphous zones con only form at the end of tincoming lifht 


\section{viii}

ion tracks when the preaccumulated concentration of point defects reaches a critical value. Depending on uniformity of the point defect distribution, two possibilities for second stage of amorphization are suggested when ion implantation is performed at different temperatures.

Diinterstitial-divacancy rairs are suggested to be the embryos of amorphous zones formed during implantation at room temperature. Silicon self-interstitials are assumed to play an essential role in the amorphization process. Out-diffusion of highly mobile interstitials during amorphization is thought to explain differences in the critical energy for amorphization with low and high energy implantation at liquid nitrogen temperature.

The proposed model represents an improved understanding of the mechanism of silicon amorphization during light ion damage at various temperatures. 


\section{INTROOUCTION}

Ion implantation has been adopted as an important step in I.C. fabrication processes for more than a decade, because it provides many advantages over thermal diffusion of impurities for doping semiconductors. Those advantages include low temperature, precise dose control, adjustable doping profiles, shallow junction, precise lateral registration and through-oxide doping. ${ }^{1-3}$ However, an inevitable disacvantage of ion implantation is the radiation damage produced by ion bombardmeni. ${ }^{4,5}$ The electrical activity of the dopant cannot be obtained unless the radiation damage is effectively repaired. Annealing after implantation, either by conventional furnace or rapid thermal annealing, is used to accomplish this purpose.

Some crystalline defects usually remain after annealing, depending on the particular condition of radiation damage and the annealing treatments. These secondary defects formed near the surface area during annealing, such as dislocation networks, stacking fau?ts, dislocaticn loops, and microtwins, are often detrimental to 1.C. device performance. ${ }^{6-8}$ control of these defects requires an understanding of their origin, i.e. the radiation damage and its recovery Juring annealing. Studies of radiation damage by ion implantation have proceeded for more than 20 years; various models ano mechanisms have been discussed. Nevertheless, many questions still cannot be answered due to the limitations of experimental techniques that have been used. However newly developed experimental techniques and wore detwiled theoretical consideration. should be able to clarify the process of 
radiation damage and phase transformation to amorphous material during ion implantation. A better understanding of these processes should lead to better control of secondary defects.

Usually there are two distinct material behaviors during annealing after implantation: recovery and recrystallization. ${ }^{9}$ Recovery is defined as a process of atomic movement from disorder back to greater order within a crystalline matrix. Recrystallization is the epitaxial regrowth of an amorphous region produced by ion bombardment; it is a phase transformation. The subject of this research is the mechanism of formation of amorphous silicon during light ion damage.

\subsection{The Importance of a Continuous Amorphous Layer}

From the study of post annealing conductance behavior of implanted layers in silicon, liquid nitrogen temperature for boron ion implantation was found to be necessary to obtain full electrical activity at low temperature $\left(-600^{\circ} \mathrm{C}\right)$ anneal. For $420^{\circ} \mathrm{C}$ or $20^{\circ} \mathrm{C}$ implantations complete electrical activity of the dopant is not achieved until the anneal ing temperature has been raised to $950^{\circ} \mathrm{C} . .^{10}$ (These curves are shown in Fig. 1 after D.E. Davies, 1969.) Low temperature annealing is very important to I.C. fabrication processes because it prevents dopant redistribution or tailing which is unavoidable for $h$ igh temperature annealing. Rutherford backscattering experiments showed that boron ions inplantation at liquid nitrogen temperature resulted in formation of an amorphous layer. 11 During the recrystallization of the amorphous layer, which can occur rapidly at $600^{\circ} \mathrm{C}$, the dopant boron atoms are incorporated into regular silicon lattice sites and become acceotors. 


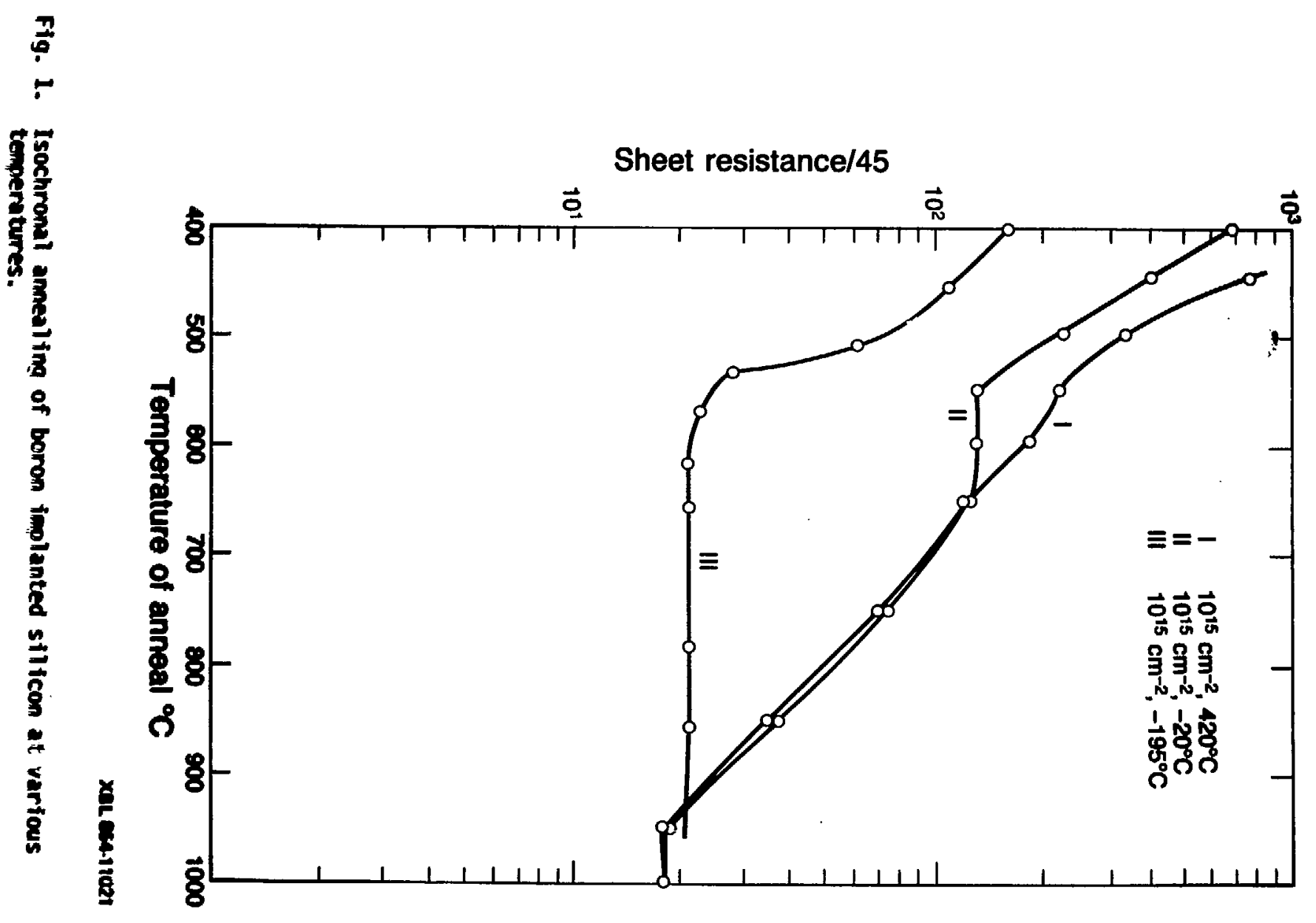


Layers implanted at $420^{\circ} \mathrm{C}$ or $20^{\circ} \mathrm{C}$ do not form a continuous amorphous layer; low temperature annealing results in high residual disorder consisting of point defect clusters and dislocation loops. The dopant incorporated into these structural defects is not electrically active. The high activation energy which is only obtained at $950^{\circ} \mathrm{C}$ is required to permit escape from these trapping centers into regular silicon substitutional sites.

The formation of secondary defects near the surface of $<100$, ion implanted silicon can be largely avoided during a rapid thermal annealing or a conventional furnace annealing, only if a continuous amorphous layer exists. ${ }^{12,13}$ The partially amorphizated or highly damaged crystalline layers resulting from ion implantation form dislocation networks, dislocation loops and stacking faults even after nigh temperature $\left(1000-1200^{\circ} \mathrm{C}\right)$ annealing. ${ }^{13,14}$ This phenomenon will be discussed later in more detail. Structural defects which penetrate certain areas of a small I.C. device can result in deleterious influences such as pipe diffusion, carrier scattering, strain fields, heterogeneous nucleation sites, and leakage currents, etc.

Continuous amorphous layer formation has become an actively investigated subject both from the theoretical point of view and the practical application view points Many authors tried to estimate or caiculate the critical dose for anorphous layer formation by proposing various models and mechanisars. 15 Also, the effects of dose rate, orientation and implentation temperature on the amorphization of ion implateri silicom have been extensively inwestigated. (refs) Heat 
dissipation during implantation is also an interesting and important topic. 16

The implantation of light ions like boron produces much less radiation damage even for similar accelerating energies as compared to a heavy ion like arsenic: boron implantation cannot form a continuous amorphous layer at roon temperature. The process of amorphization by light ion damage in silicon is oifferent from the amorphization by heavy ions, the latter has been reasonably well understood for some time while the former is still unciear in nature. This work attempts to clarify on an atomic level the mechanism of light ion amorphization.

\subsection{Nuclear and Electronic Stopping}

In general, the energy loss when an accelerated ion penetrates into a target consists mainly of nuclear stopping and electronic stopping. The reason why a light ion causes less radiation damage is not because a light ion cannot bring the same energy as the heavy ion, but it is because most of the light ion's energy is consumed by eiectronic stopping during penetration into the target. Electronic stopping is associated with interactions between the electrons of the noving ion and the electrons in the target. Electronic stopping cannot produce crystal lattice damage unless the implantation reaches a threshold energy, which was calculated as 802 meV for boron, c2.50 Meir for phosphorus and 5470 keV for arsenic fons in a silicon target using relativistic kinetics framework. 17 ion inplantation usually eaploys acceleration under $200 \mathrm{kel}$ volizage. In this range of accelerating voltage, electronic stopping cannot displace stlificon lattice atoms, 
it can only ionize the atons and eventually degrade into heat which is dissipated.

Nuclear stopping which is the screened coulomb collisions between the moving ion and the target atoms, does displace silicon lattice atoms and produces Frenkel pairs ( $v$ ancy and interstitial) in the crystalline matrix. ${ }^{18}$ Therefore, the degree of radiation damage is dependent on the percentage of nuclear stopping in the whole energy loss process for an incoming ion.

Linchard, Scharff and Schiott ${ }^{19,20}$ (referred to subsequently as LSS) have developed a universal relationship for the nuclear stopping $\left(d_{\varepsilon} / d_{p}\right)$ n and electronic stopping ( $\left.d_{\varepsilon} / d_{\rho}\right)$ e in terms of dimensionless length and energy parameters, defined as

$$
\left.\rho=R \pi a^{2} N\left[, M_{1} / M_{1}+M_{2}\right)^{2}\right]
$$

and

$$
\varepsilon=E\left(a / e^{2}\right)\left[M_{2} / Z_{1} Z_{2}\left(M_{1}+M_{2}\right)\right]
$$

where

$$
a=0.8853 a_{0} /\left(z_{1}^{1 / 2}+z_{2}^{2 / 3}\right)^{1 / 2}
$$

$R$ is the vaive of the ion penetration range in the unit of micron, $E$ is the value of the ion energy in the unit of $\mathrm{KeV}, a_{0}$ is the Bohr radius $\left(0.529 \times 10^{-8} \mathrm{~cm}\right), N$ is the number of atoms per unit volume, $Z$ and $M$ are the atomic number and mass, and the subscripts 1 and 2 refer to the projectile and target atoms respectively. The resulting universal relationship between $(-d c / d /)_{n}$ and $e^{1 / 2}$ is shom by the 
solid line in Fig. 2. ( $\varepsilon^{1 / 2}$ is proportional to ion velocity.) The dot-dash line in Fig. 2 repreșents the electronic stopping power $(-d \varepsilon / d o)_{e}$ given by

$$
(-d \varepsilon / d \rho) e=K \varepsilon^{1 / 2}
$$

where

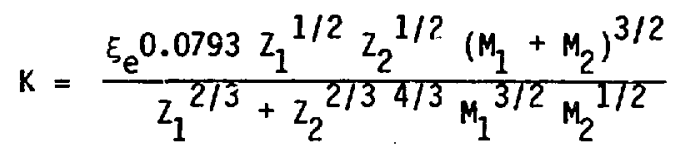

and $\xi_{e}$ is of the order of 1 to $2\left(\xi_{e} \simeq z_{1}^{1 / 6}\right)$. The electronic stopping calculations therefore do not produce a universal $\left(-d_{\varepsilon} / d_{\rho}\right)$ e curve, but rather a set of curves each characterized by a particular $k$ value. (The values of $k$ is between 0.1 and 0.25 for most of the situations.)

In Fig. 2, there is a maximum value for nuclear stopping at $\varepsilon_{1}$ (where $\varepsilon_{1}$ is around $\varepsilon=0.35$ ), a maximum value of electronic siopping at $\varepsilon_{3}$, and a transition point $\varepsilon_{2}$ at which nuclear and electronic stopping become equal. Table 1 shows those characteristic energies (in KeV) corresporiding to $\varepsilon_{1}, \varepsilon_{2}$ and $\varepsilon_{3}$ for $B, P, A s, S b, B i$ in the targets of $\mathrm{Si}, \mathrm{Ge}$ and $\mathrm{Sn} .{ }^{21}$ The energy loss of an incoming ion is dominated by electronic stopping initially, if the energy of the accelerating ion is larger than the $c_{2}$ energy. When the ion has slowed down during penetration of the target to an energy below $c_{2}$, then nuclear stopping starts to dominate the energy loss process; in other words, the incoming ion increasingly produces structural radiation damage. 


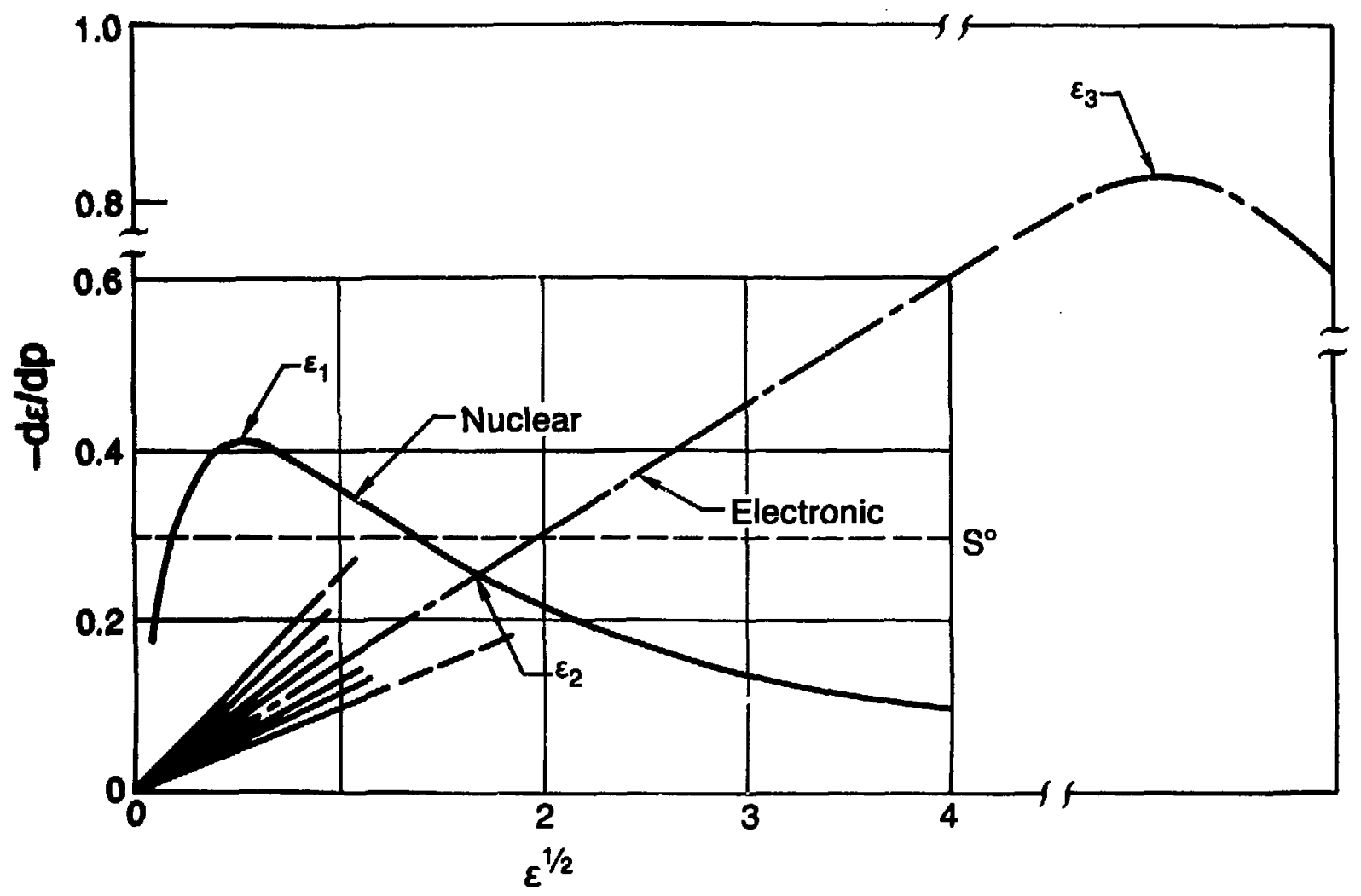

F4g. 2. Theoretical nuclear and electronic stopping-power curves expressed in terms of the reduced variables $\rho$ and $\varepsilon$ (based on LSS). For electronic stopping, a family of IInes is obtained (one for each combination of projectile and target). The dotdash line represents the electronic stopping for $K=0.15$. The horizontal line labeled $\mathrm{S}^{0}$ represents the constant-stopping-power approximation suggested by Nielsen. 
Table 1.

Characteristic Energies (in KeV)

Corresponding to $\varepsilon_{1}, \varepsilon_{2}$, and $\varepsilon_{3}$ in Figure 2

\begin{tabular}{|c|c|c|c|c|c|c|c|}
\hline \multirow{2}{*}{ Ion } & \multicolumn{3}{|c|}{$\varepsilon_{1}$} & \multicolumn{3}{|c|}{$\varepsilon_{2}$} & \multirow[t]{2}{*}{$\varepsilon_{3}$} \\
\hline & in Si & in Ge & in $5 n$ & in Si & in Ge & in $5 n$ & \\
\hline B & 3 & 7 & 12 & 17 & 13 & 10 & $3 \times 10^{3}$ \\
\hline $\mathbf{P}$ & 17 & 29 & 45 & 140 & 140 & 130 & $3 \times 10^{4}$ \\
\hline As & 73 & 103 & 140 & 800 & 800 & 800 & $2 \times 10^{5}$ \\
\hline Sb & 180 & 230 & 290 & 2000 & 2000 & 2000 & $6 \times 10^{5}$ \\
\hline $\mathrm{Bi}$ & 530 & 600 & 700 & 6000 & 6000 & 6000 & $2 \times 10^{6}$ \\
\hline
\end{tabular}

\subsection{Radiation Damage by Heavy Ion and Light Ion}

The physical meaning of the LSS universal relationship (Fig. 2) can be interpreted using the concept of scattering cross section during a collision. The scattering cross section of the nucleus decreases with increasing incoming ion velocity. This implies that the electronic stopping dominates the energy loss prosess when the velocity or energy of the incoming ion is still high. The scaitering cross section increases with increasing mass of the ions. Therefore $c_{2}$ increases with ion mass. For example, for a heavy ion such as arsenic with an atomic mass of $75, c_{2}$ is 800 KeV (shom in Table 1). Therefore the energy loss for an incoming arsenic ion with a few hundred KeV acceleration energy is dominated by nuclear stopping along 
the entire path. On the contrary, the $\varepsilon_{2}$ energy of a light ion such as boron (atomic mass 11) is $17 \mathrm{keV}$ (shown in Table 1). Most of the energy loss of an incoming $100 \mathrm{KeV}$ boron ion is dominated by electronic stopping except near the end of the track. Consequently, when boron ions have a significant probability of collision with the target atoms, they already have lost most of their initial energy, only $-10 \mathrm{keV}$ remains. The number of silicon atoms displaced by each light ion is therefore much smaller than that due to a heavy ion with the same initial energy. Also the separation distance between the vacancy and the knocked out atom is generally shorter in the light ion damage case, therefore recombination of the vacancy and the interstitial is more probable for light ion damage than for heavy ion damage.

To take a somewhat simplified example, a heavy Sb ion implanteo into a silicon target with $100 \mathrm{KeV}$ can create a high density disordered zone with roughly cylindrical shape of $25 \AA$ radius and $500 \AA$ length. The average nuclear stopping of a $100 \mathrm{KeV}$ Sb projectile in a silicon target is about $0.2 \mathrm{KeV} / \AA^{2},{ }^{22}$ and the average distance between lattice planes in silicon is about $2.5 \AA$. The Sb projectile would lose about $500 \mathrm{eV}$ per lattice plane. If the majority of this energy is given to one primary silicon knock-on aton, then the average range of the recoileo atom from the center of the ion track is about $25 \AA$, using the average nuclear stopping of $\mathrm{Si}$ in $\mathrm{Si}$ as $20 \mathrm{eV} / \mathrm{A}$. By using Kinchin and Pease's nodel, 23

$$
N_{d}=E_{12 E_{d}}
$$


where $E$ is the projectile energy, $E_{d}$ is the displacement energy for a target atom, which is about $15 \mathrm{eV}$, and $N_{d}$ is the number of target atoms displaced by a primary recoil, there are roughly 15 displacements per lattice plane with in the cylinder zone, or 3000 displacements totally. The displacement density is about .20 atomic fraction in silicon. This density of point defects is thought to be high enough to make the crystalline structure unstable and a transformation to the amorphous state spontaneously occurs during ion implantation. Recent calculations have suggested that a density of Frenkel pairs of only 10 at $77^{\circ} \mathrm{K}$ is sufficient for amorphization (Christel et al)..$^{24}$ Each incoming Sb ion should produce enough localized damage to form an individual amorphous zone extending from the surface to a depth of $500 \AA$. This proress is schematically drawn in Fig. 3(a). Evidence of amorphous zone formation by an individual heavy ion has been obtained by $h$ igh resolution TEM. 25

For a light ion such as boron with $100 \mathrm{Kel}$ energy implanted into silicon, only about 200 displacements would be distributed over the first $2000 \AA$ of the ion path, and 500 displacements would occur in the last $2000 \AA$ of the ion path. Frenkel pairs resulting from this damage either recombine or form point defect clusters which are stable and immobile at room temperature. No anorphous zone can be formed by an individual boron ion initially. The reasons for the difference of the radiation damage caused by a heavy ion and a light ion can be sumarized as 1) a !ight ion loses a substantial fraction of its total energy in electronic stopping; 2) primary recoil atoms are produced 
(a)

Incoming Heavy lon

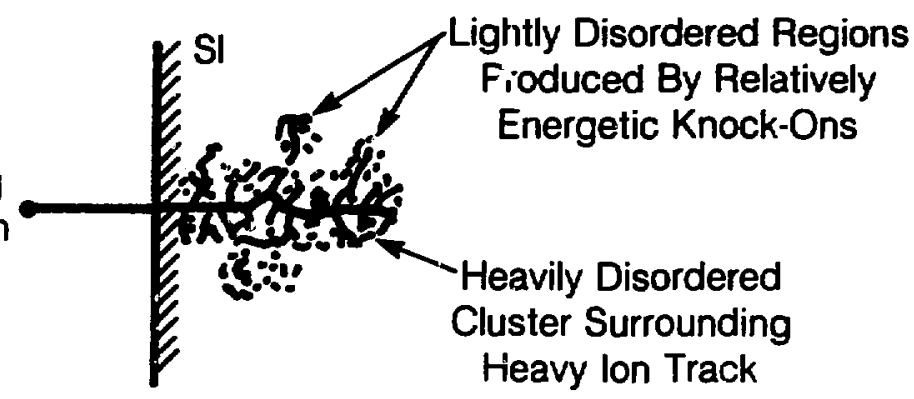

(b)

Incoming

Light lon

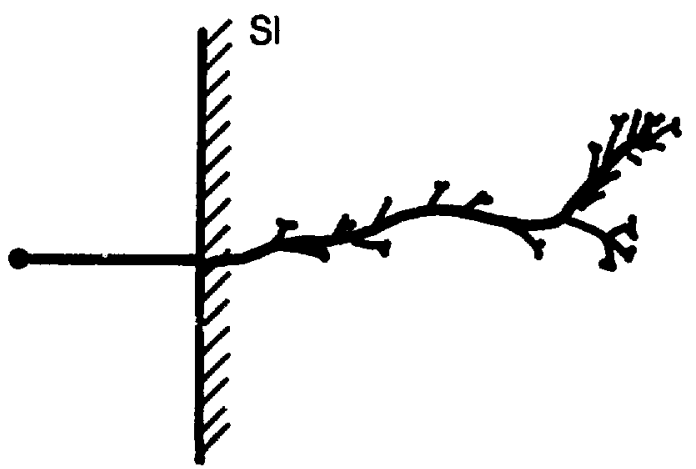

XBL 8510-11718

Fig. 3. Illustrating the damage produced by a heavy ion (a) and a light ion (b). 
with much less energy and therefore create much less damage; and 3) the range of the light ion (4000 A for a boron ion with $100 \mathrm{KeV}$ ) is much larger than that of a heavy ion with the same initial energy. The typical light ion damage process is schematically shown in Fig. $3(b)$.

\subsection{Heterogeneous and Homogeneous Nucleation}

The heterogeneous nucleation model has been used to describe amorphization by heavy ion implantation at low temperature, while the homogeneous nucleation model has been used to describe amorphization by light ion implantation at high temperature. Heterogeneous nucleation assumes that an amorphous zone is formed spontaneousily at the end of each incoming ion track, and a continuous amorphous layer is formed by the overlapping of those amorphous zones. The homogeneous nucleation model is described as follows: when the accumulation of point defects and small clusters formed through diffusion processes reaches a critical value, the crystalline structure is no longer stable, it then suddenly collapses into the amorphous state.

The heterogenous nucleation model has successfully explained the amorphization process for heavy ion implantation through the prediction of amorphizing doses for different heavy cons (Morehead and Crowder's theory). ${ }^{26}$ However, this model does not provide a reasonable explanation for the anorphization by light ion damage. A disorder over lapping model suggested by Gibbons ${ }^{27}$ modified the homogeneous nucleation model in order to explain the amorphization by light ions. Wevertheless, the assupstion of mobile point defects in this 
model is still unreasonable for light ion implantation at room temperature at which the simple point defects are very mobile.

The homogeneous nucleation model gives a good description for the initial stage of light ion damage, but it iacks an explanation of how the accumulated point defect clusters are eventually transformed to the amorphous state. Besides, it does not satisfactorily explain the two stage change in optical characteristics during the amorphization process by 1 ight ion damage. 28

\subsection{Two Stage Phenomenon in IR Reflection}

Baranova et al. conducted an infrared reflection experiment near a fundamental absorption edge to study the relationship of amorphous silicon formation with increasing ion fluence. ${ }^{26}$ They found an important trend for this relationship by decreasing the ion mass from $\mathrm{Sb}^{121}$ to $\mathrm{B}^{11}$. The results of this experiment is shown in Fig. 4 . The $y$ axis of the curve is represented by the relative increment of refractive index

$$
\frac{n_{-n}}{n_{0}}
$$

where $n$ is the refractive index of ion implanted silicon.

$n_{0}$ is the refractive index of unc. anged silicon.

The refractive index is a parameter which represents the dielectric property of material. The refractive indices of crystalline silicon and amorphous silicon are significantly different. Hence the amount of amorphous silicon formed during amorphization of a silicon surface 


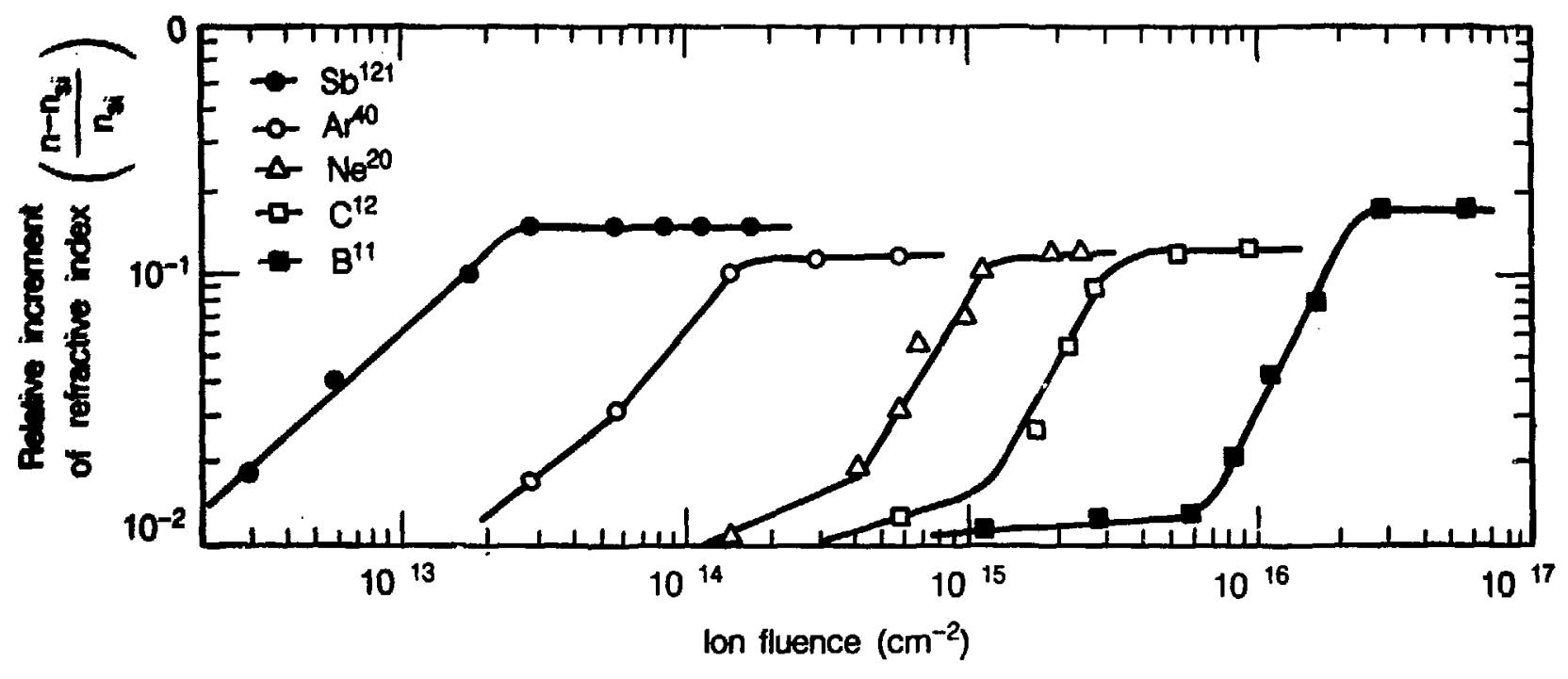

Fig. 4. Relative change of the refractive index near fundamental absorption edge $(\lambda=1 \sim 2 \mu)$ for silicon irradiated with $80 \mathrm{keV}$ ions of different species. 
layer by ion implantation was considered to be proportionally represented by the relative increase of refractive index measured in the $I R$ reflection experiment. The $X$ axis of the curve ropresents the ion dose implanced into silicon at room temperature with $80 \mathrm{KeV}$ energy and a dose rate lower than $1 \mu \mathrm{A} \mathrm{cm}^{-2}$.

Ions of different mass, $\mathrm{Sb}^{121}, \mathrm{Ar}^{40}, \mathrm{Ne}^{20}, \mathrm{C}^{12}$, and $\mathrm{B}^{11}$ were used in this experiment. The heavy ion $\mathrm{Sb}$ showed a single increasing stage before saturation at which a continuous amorphous layer was supposedly formed so that the refractive index change cannot increase with further ion dose. The lighter ions starting from Ar to B all showed two stages increasing with lower slope in the first stage and with a higher slope in the second stage during continuously increasing ion bombardment (Fig. 4). The slopes of the first and second stage in the curve for Ar were very close. It is suggested that a heavier ion than Ar would have only one single stage like $\mathrm{Sb}$. The difference of the slopes at these two stages increases with decreasing ion mass. Boron ion which was the lightest ion in the experiment has the most pronounced difference for these two stages. It is suggested from this two stage characteristic that the amorphization of silicon by light ion implantation at room temperature could also consist of two stages with accumulation of point oefects in the first stage and amorphous silicon formation in the second stage.

The homogeneous nucleation model can explain the behavior for light ion damage of silicon ouring the first stage, but does not explain the slope of the second stage or the sudden change in slope. 
If the oamaged crystalline material would suddenly transform to amorphous material when the critical concentration of point defects had been accumulated, then the relative refractive index might be expected to show a rapid rise at the end of stage 1 . However, this is not the case as shown by the IR refiection experiment. A more detailed mechanism for the second stage of light ion damage needs to be developed to fit these resuits.

\subsection{The Present Work}

The objective of the present work is to obtain a better understanding to the amorphization mechanism for light ion damage in silicon for low temperature and room temperature. In order to achieve this purpose, the structure and properties of amorphous silicon will be reviewed first to provide a basis for discussion of the crystalline to amorphous transformation. Then the various mechanisms and models concerning silicon amorphization by ion implantation which have been previousiy developed will be discussed and commented on to identify the essential questions which still need to be answered by an improved amorphization model. All this will be included in Chapter 2. An amorphization model which is based on previous experimental results and theoretical considerations will be proposed for boron ion implanted into silicon at room temperature in chapter 3. Depending on the uniformity of the accumulated point defect cluster distribution, two possibilities for the second stage of amorphization are suggested for the ion implantation at different temperatures. The accumulation of point gefects and/or complexes is constaered to be required during the 
initial stage of the amorphization process. Divacancies and di interstitials which are the smallest point defects stable at room temperature, are suggested to be uniformly distributed to form the necessary amorphous embryo pairs. Energy transfer from incoming light ions is thought to be required for reorganization of bonds during the phase transformation, crystal to amorphous.

Experimental approaches which include using high resolution cross section TEM (Transmission Electronic Microscope) and qualitative EPR (Electron Paramagnetic Resonance) techniques, are designed to obtain more evidence to clarify the true amorphization mechanism. The formation of amorphous zones by light ion damage is demonstrated for the first time in these experiments. The effects of implantation temperature :nd dose rate on the amorphization will be shown and discussed in Chapters 6 and 7. A liquid nitrogen temperature or high dose rate implantation is found to be necessary for the formation of a continuous amorphous layer in a borion ion implanted silicon wafer. It is suggested that out diffusion of silicon interstitials during ion implantation can explain the lowering of critical deposited energy for amorphization for MeV high energy implantation.

Instead of continuous amorphous layer, a discontinuous partial amorphous layer with faulted dislocation loops in the remaining crystalline regions was formed by high dose boron ion implantation at room temperature. These faulted loops which represent large clusters of silicon interstitials, cen occur only after room temperature implantation. This not only shows the evidence of the point defects 
aggregation during implantation, it aiso interprets the cause of the secondary defects formation.

A new and more detailed amorphization model for iight ion damage is presented here, combined with critical experimental resuits. 


\section{AMORPHOUS SILICON AMD MORPHIZATIOA}

\subsection{Amorphous Silicon}

In oraer to study anorphization it is necessary to have a model of the amorphous state, especially in the sense of microstructure and thermodynamics. Amorphous semiconductors have been a hot topic of investigation in recent years due to their important appiications but the theoretical understanding of these disordered systems is still larg-ly undeveloped. ${ }^{29}$ Fortunately, amorphous silicon is the most extensively studied material. Here, the structure for an ideal amorphous silicon will be discussed and then it will be mo ified to fit the experimental resulte The thermodynamic criterion for the crystailine-amorphous transition will be based on experimental results on polycrystalline deposition. A distinction between amorphous and glassy materials will also be briefly discussed.

\subsubsection{The structure of amorphous silicon.}

A tetrahedrally coordinated random-network structure was constructed as an idealized model of the: structure of amorphous $\mathrm{Si}$ or $\mathrm{Ge}$ by Polk in $1971,{ }^{30}$ based on the concepts of Zachariasen. ${ }^{31}$ In such a structure, each atom has four neighbors in an approximately tetraredral arrangement; noncrystallinity is achieved through variations in the tetrahedral angle and through the relative rotation of adjoining tetrahedre into configurations other than the configuration found in the aiamond cubic structure. Considering the strong covalent bonding of Si, a madel with no significant variation in the first-neighbor aistances was cesired. Computer itrations of the arrangement of atom 
positions was used to reduce the standard deviation of the firstneighbor distance in the constructed physical model..$^{32}$ With the atomic radius of 40 , the mean bond iength of the refined model was 7.9403 with a standard deviation of $0.0133(0.168)$. The mean bond angle was $109.2^{\circ}$ with a standard deviation of $9.1^{\circ}$. The radial distribution function (RDF), $4 \pi r^{2} \rho(r)$, of the Polk-Boudreaux tetrahedrally coordinated random network structure was computed and is shown in Fig. 5 with a comparison of the RDF of a diamond cubic crystalline structure. ${ }^{33}$

In a diamond cubic structure, each atom has 4 nearest neighbors tetrahearally arranged, 12 second-nearest neighbors at 1.63 times the nearest neighbor distance (NND), and 12 third-nearest neighbors at 1.92 NND, as indicated in Fig. 5. With the top view of diamond cubic structure in [111] direction, it is easily seen on Fig. 6(a) that the layers are composed of puckered six-fold rings in the "chair" configuration with the puckered atoms whose fourth bond connected to the above layer being located $1 / 3$ NND above those whose fourth bond connected to the layer below. The "chair" configuration can also be easily seen in the direction tilting a few degrees from [110], as shown in Fig. 6(b). The entire crystal can be thought of as composed of these chair-type rings.

The longest distance between atoms in one particular $r$ ing is the thira-nearest neighbor distance (1.92 N.N.O). In Fig. 5, the first and second peaks of the idealized amorphous radial distribution function are similar to those of the crystal : 4 nearest neighbors at 


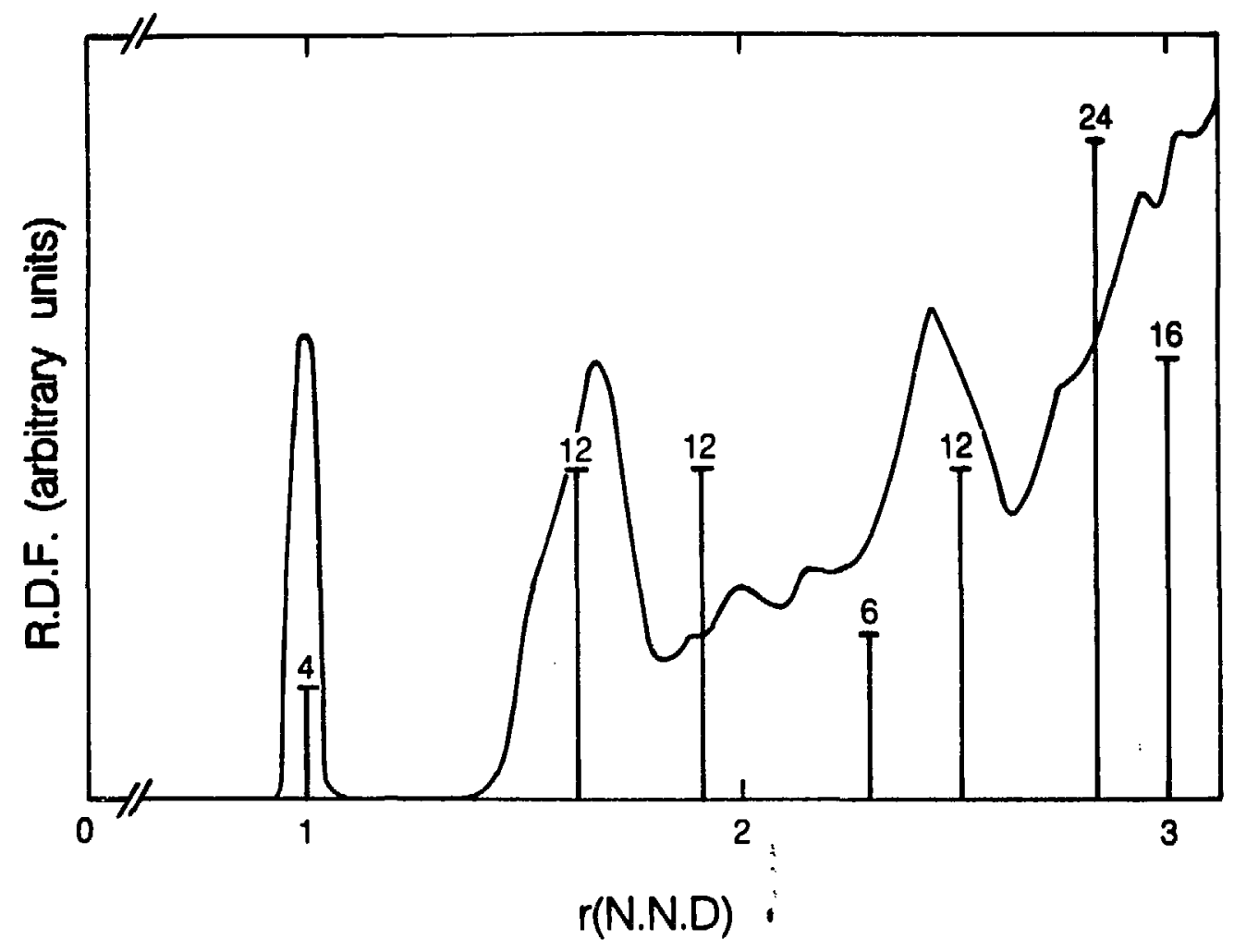

XBL 8510-11622

Fig. 5. Radial distribution function of the Polk-Boudreaux tetrahedrally coordinated random network structure. The number and positions of neighbors in the diamond cubic lattice are indicated by the vertical 1 ines. 
(a)

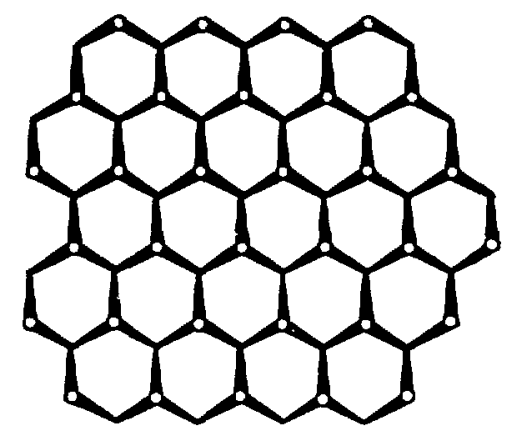

(b)

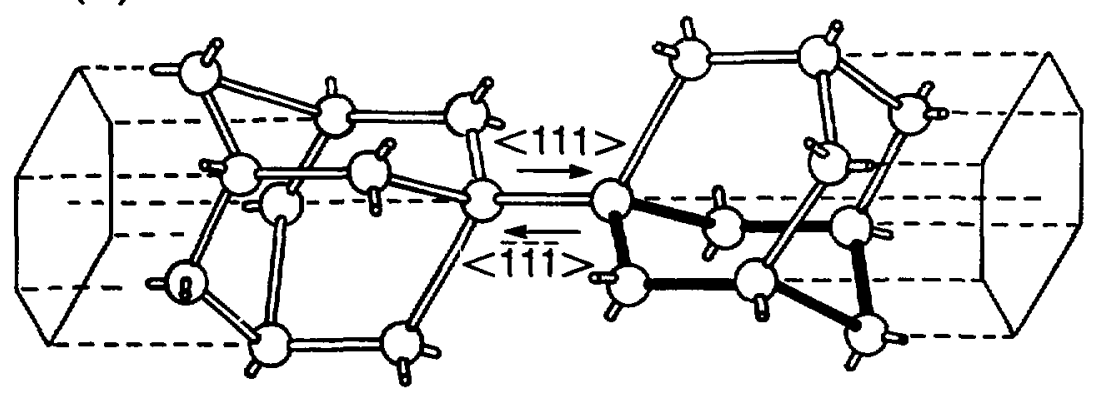

$X B i$ 8510-11621

Fig. 6. (a) Topology of diamond cubic structure in [111] direction. (b) Chair configuration of puckered six-fold rings in diamond cubic structure. 
1 N.H.D, and 12 next nearest neighbors spread around 1.63 N.N.D at distance aepending on the bond angle distortion. The essential difference between the two distributions is the third peak at 1.92 N.N.D, which is very strong in the crystal but almost totally smeared out in the idealized amorphous structure. As mentioned above, this distance corresponds to the diagonal distance in the chair-type six fold rings which make up the crystal. The idealized amorphous structure, on the other hand, is made of a mixture of five to eight fold rings, in various proportions, shapes or degrees of distorted tetrahedral bonds. The mixtures of five, seven and eight fold rings replacing most of the chis r-type sixfold rings were constructed continuously without making dangling bonds to form a random-network structure, which is $1.0 \%$ less dense than a diamond cubic structure. ${ }^{32}$ This $k$ ind of continuous randori-network structure was considered satisfactorily to be close to the structure of amorphous $S i$ or Ge in the first half of the last decade. ${ }^{34-36}$ Several models of the similar type have been built either using plastic tetrahedral units or computer simulation with an agreement of the characteristics of density close to that of the crystal, no dangling bonds, bond angle distortion less than $20^{\circ}$ ( $r$.m.s $\left.-10^{\circ}\right)$ and a radial distribution function similar to one in Fig. $5 .^{37-41}$

However, the relatively low energy bond angle variation are not sufficient to accomodate all network strains. ${ }^{42}$ A pure amorphous silicon was considered highly defective ${ }^{43}$ having $-10^{20}$ paramagnetic states per $\mathrm{cm}^{3414}$ and an abundance of internal surfaces. A measured 
diffuse $X$ ray scattering spectrum for amorphous silicon was compared to the calculated positions and width of the 220 and 311 paaks of polycrystalline silicon by Veprek et al. 45 as shown in Fig. 7 . The second maximum in the diffuse scattering from amorphous silicon materials does not correspond to super position of the 220 and 311 peaks, when the first peak of the measured curve is interpreted as the 111 Bragg reflection. Hence, the real structure of amorphous silicon is slightly different from the idealized structure of amorphous silicon as a continuous random-network, but is a similar structure which has less tetrahedral angle distortion and dangling bonds with a density of about $10^{20}$ per $\mathrm{cm}^{3}$. The strong potential minimum at the optimal bond length ${ }^{46}$ produces a system in which the total energy of the network can be minimized by localizing strains in broken bonds. 47 ESR (Electron Spin Resonance) or EPR (Electron Paramagnetic Resonance) was one of the earliest experimental tools applied to the study of dangling bonds in amorphous silicon. A characteristic signature, the inhomogeneous broadened resonance with a g-value of 2.0055 is the only signal seen in undoped amorphous silicon independent of preparation condition. $44,48-50$ The density of these centers depends strongly on preparation conditions, varying from $-10^{20} \mathrm{~cm}^{-3}$ for pure amorphous silicon to $\leq 10^{15} \mathrm{~cm}^{-3}$ for a-SiH (hydrogenated anorphous silicon). ${ }^{43}$ The axorphous resonance peak appears isotropically due to the random orientations of the dangling bonds in amorphous silicon. Because of the existence of the dangling bonds in the real anorphous silicon structure, the second peak in the $x$-ray scattering of anorphous 


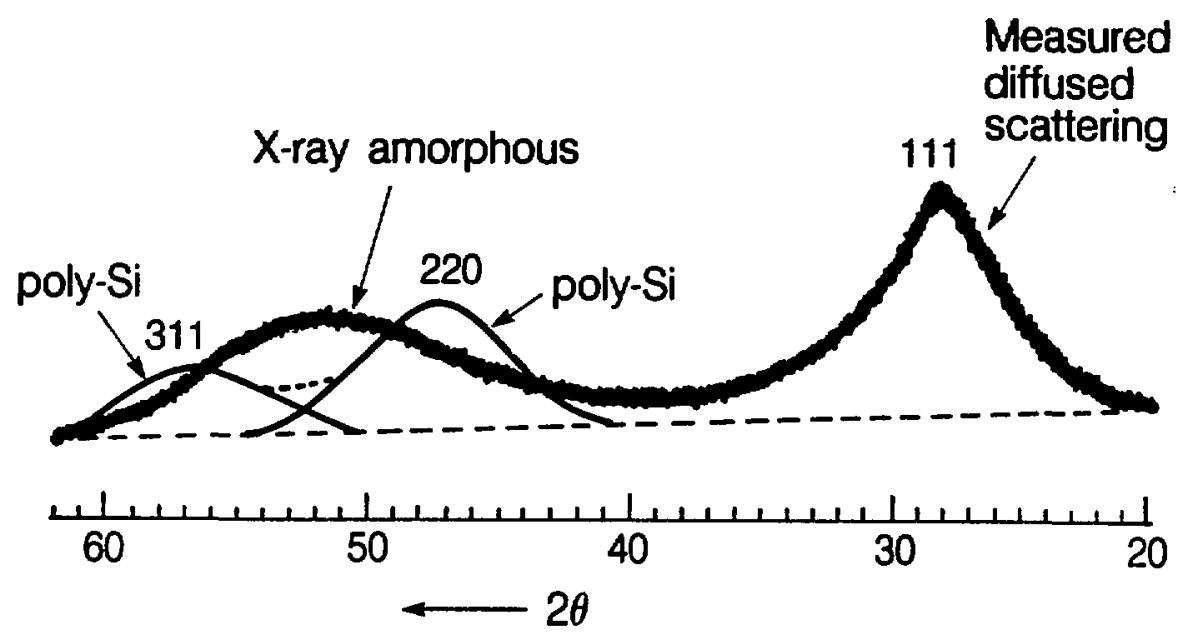

XBL 8510-11623

Fig. 7. A part of the measured diffuse $x$-ray scattering from amorphous $\mathrm{Si}$ and the calculated positions and widths of the 220 and 311 peaks of crystalline $S i$ when the first peak of the measured curve is interpreted as the 111 Bragg reflection. 
silicon is located between the second and third nearest neighbor distance (220 and 113 reflection peaks) in a diamond cubic lattice (Fig. 7); when the second peak of the R.D.F. for the idealized amorphous structure is consistent with the location of -second N.N.D. in the silicon crystal.

\subsubsection{Thermodynamics criterion for the crystalline-amorphous transition.}

The amorphous structure was generally considered as a structure with a short range order due to the strong covalent bonds in the solid state. A polycrystal with very small grain sizes $(30 \AA \sim 100 \AA)$ can also be considered as a structure of only short range order. Veprek et al.$^{51}$ has shown that the crystalline-to-amorphous transition for deposited silicon can be controlled through the deposition parameters, which essentially governs the lattice expansion. At a certain limit of the linear lattice expansion $\Delta d / d_{0} \simeq 0.01-0.02$, the diamond cubic lattice becomes unstable and the amorphous phase is formed. This is illustrated by the solid line curve and the full circles in Fig. 8. Experimental results of the effect of compressive stress on the stabilization of the diamond lattice are also shown in Fig. 8 with the open circies. In the stress-free films the lower limit of the crystalline size is $-30 \AA$, but it can be decreased to $-20 \AA$ for $f i l m$ possessing a large compressive stress. 45 The polycrystalline silicon films were deposited on various substrates such as silica and silicate glass, etc. in a d.c. hydrogen plasma at low pressure. The crystalline size has been evaluated from the width of the (111) and 


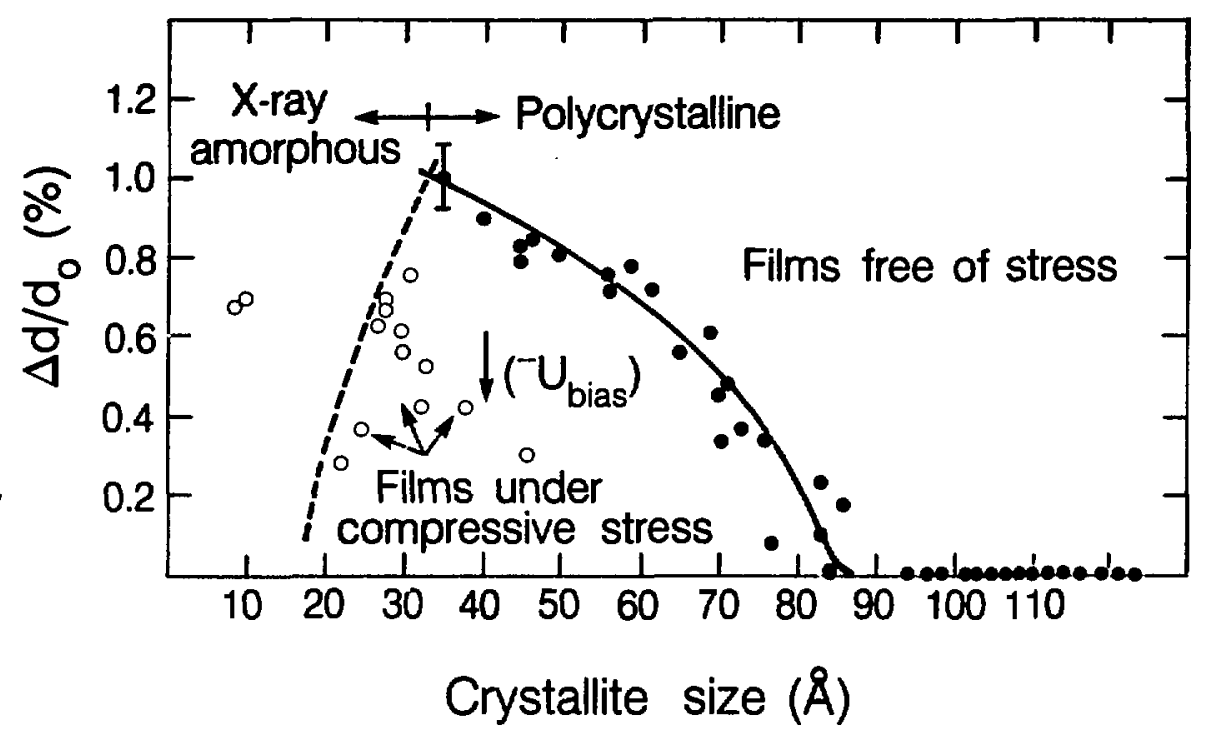

XBL $8510-11624$

Fig. 8. Correlation betweer the measured lattice expansion, $\Delta d / d_{0}$ and the crystalline size, for nearly stress-free films (full) circles and solid lines) and for films deposited under negative bias, i.e., films having a compressive lattice stress produced by ion-implantation (open circles). 
(220) peaks using Scherre formula $\mathrm{a}^{52}$ with a shape factor of 0.94 . The grain size of the deposited microcrystalline silicon decreases when the deposition temperature decreases. A compress $i$ state for the deposited film was obtained if a negative bias was applied to the sample holder by means of an auxiliary power supply. 51

With decreasing crystalline size the contribution of grain boundary energy to the free energy of the system, $F(\mu c-S i)$, increases. The excess energy, $\delta \mathrm{F}(\mu \mathrm{C}-\mathrm{Si})$, can be split into two parts:

$$
\delta F(\mu c-S i)=\delta F(b u i k)+\delta F(S i-H)
$$

$\delta \mathrm{F}(\mathrm{Si}-\mathrm{H})$ represents the contribution to the free energy of the system from the chemically bonded hydrogen. The first term on the right-hand side of the above equation is approximately equal to the elastic energy due to the lattice expansion since, as long as the diamond lattice is preserved, the entropy term of of(bulk) can be neglected. Thus $\delta F\left(\right.$ bulk) can be expressed by the elastic energy density, $U\left(\Delta d / d_{0}\right),{ }^{53}$

$$
\delta F(\text { bulk })=\bar{V}_{\text {mole }} U\left(\frac{\Delta d}{d_{0}}\right)=\frac{\bar{V}_{\text {mole }} e^{B}}{2}\left(\frac{\Delta V}{V}\right)^{2}
$$

Where $y_{\text {mole }}=12 \mathrm{~cm}^{3}$ is the molar volume of solid silicon, $B=0.99 \times 10^{12}$ dyne $\mathrm{cm}^{-2}$ is the bulk modulus and $\Delta V / V=3\left(\Delta d / d_{0}\right)$ is the volume expansion. Hence, the free energy of ${ }_{\mu} \mathrm{C}-\mathrm{Si}$ can be given by:

$$
F(\mu C-S i)=E O-T S O+(9 / 2) V_{\text {mole }} B\left(\delta d / d_{0}\right)^{2}+\delta F(S i-H)
$$

where Eo and So are the molar internal energy and entropy of silicon 
single crystal. In a similar way, the free energy of amorphous silicon is given by

$$
F(a-S i)=E 0+\delta E(a)-T(S O+\delta S(a))+\delta F(S i-H)
$$

where $\delta E(a)$ and $\delta S(a)$ stand for the excess configurational internal energy and entropy respectively. $\delta^{\prime} F(S i-H)$ is the term for the energy contribution from hydrogen bonding in amorphous silicon. When the crystalline to amorphous transition occurs $\delta \mathrm{F}(\mathrm{Si}-\mathrm{H})$ is approximately equal to $\delta F^{\prime}(S i-H)$, thus:

$$
(9 / 2) V_{\text {mole }} B\left(\Delta d / d_{0}\right)^{2}=\delta E(a)-T \delta S(a)
$$

The excess internal energy for amorphous silicon was estimated as $\delta E(a)=0.1-0.6 \mathrm{Kcal}_{\mathrm{mol}}^{-1} \cdot{ }^{54-56}$ The upper 7 imit of the configuration entropy for amorphous silicon was calculated as $\delta S(a)=0.4 \mathrm{Cal}$ $\mathrm{K}^{-1} \mathrm{~mol}^{-1}$ by Spaepen. ${ }^{57}$ Using these values, the critical lattice expansion at which the diamond lattice becomes unstable with respect to the amorphous state can be estimated to be

$$
\left(\frac{\Delta d}{d}\right)_{\text {critical }}=0.01-0.02
$$

This is in good agreement with the experimental results of the lattice expansion of the polycrystalline silicon with a minimum grain size of $30 \mathrm{~A}$ before the transition. Comparing the density of the continuous random nethork for the idealized anorphous silicon structure wich is 17 less than the density of the crystal, the data of 1-28 dilatation for amorphous silicon appears to be reasonable. 


\subsubsection{The first order phase transition.}

The aiscontinucus nature of the crystalline to amorphous transition is evident from the following experimental observations.

(1) High resolution TEM lattice images showed an atomically sharp interface between amorphous regions and crystalline regions. 25,58

(2) The change in the $x$-ray pattern (Fig. 7) is discontinuous regardless of whether the crystalline to amorphous transition occurs by a low temperature deposition ${ }^{51}$ or by ion implantation. 59

(3) The Raman spectrum shows an abrupt disappearance of the crystalline $\mathrm{T}_{\mu_{25}}$, mode below the lower crystallite size limit. ${ }^{60}$

(4) The electrical conductivity of Si and Ge decreases sharply upon the transition to the amorphous phase. ${ }^{61}$

(5) The sign of the Hall mobility changes from the normal to the anomalous one upon transition. 62

In addition, it has been recognized since $1975^{63}$ that there exists a discontinuity of the coherent length on going from polycrystalline to the amorphous material. All the above phenomena suggest that the crystalline to amorphous transition is a first order phase transition.

Landau's theory of phase transitions states that "if there is no common subgroup of the symmetry groups of both phases, the transition between them has to be discontinuous." Th statexent also inplies that the transition for Si and Ge would be first order. However, if, as in the case of good glass-formers, clusters with certain symetry elements of the crystalline phase exist, the crystalline to noncrystalline transition should be second order. The fundamental difference 
between the two main classes of noncrystalline semiconductors, glasses and amorphous films, has been discussed by Fritzsche. ${ }^{64}$ The main difference is essentially due to the mismatch between bonding contrains and the number of degrees of freedom in three dimensions, and to the flexibility required to accommodate the mismatch. Phillips has treated this difference by the parameter of average conrdination number, $m$, which is considered in the case of a binary alloy $A_{x} B_{1-x}: 65$

$$
M=X N_{c n}(A)+(1-X) N_{c n}(B)
$$

The number of constraints $N_{\text {co }}$ per atom is given by

$$
N_{c o}(m)=m / 2+m(m-1) / 2=m^{2} / 2 \text {. }
$$

The first term on the middle of the above equation is given by the bond stretching interaction and the second term by the bond-bending interactions which are assumed for simplicity to be equal for the A and $B$ atoms. A schematic figure for the classification of noncrystalline solids is shown in:Fig. 9. The mean coordination $m=4$ separates noncrystalline metals from semiconductor or insulator. Materials with higher connectivity $4 \geq m \geq 3$ are overconstr ained amorphous to which amorphous Si and Ge belong. Glasses are normally restrirted to $3 \geq m>2$. Those having lower connectivity $m<2$ are under crosslinked amorphous, such as amorphous films of $I_{2}, B r_{2}$ and inert gases. Considering that the number of constrains for glasses is equai to the three spatiat degrees of freedom, $N_{c o}=3$, thus

$$
a_{c}=\sqrt{6}=2.45
$$

is the aver aje coordination corresponding to an optimal glass-forming conmectivity; the average coordimation number a of morphous silicon is approxtimaitely equal to the to the strong tetrabedral bondings. 


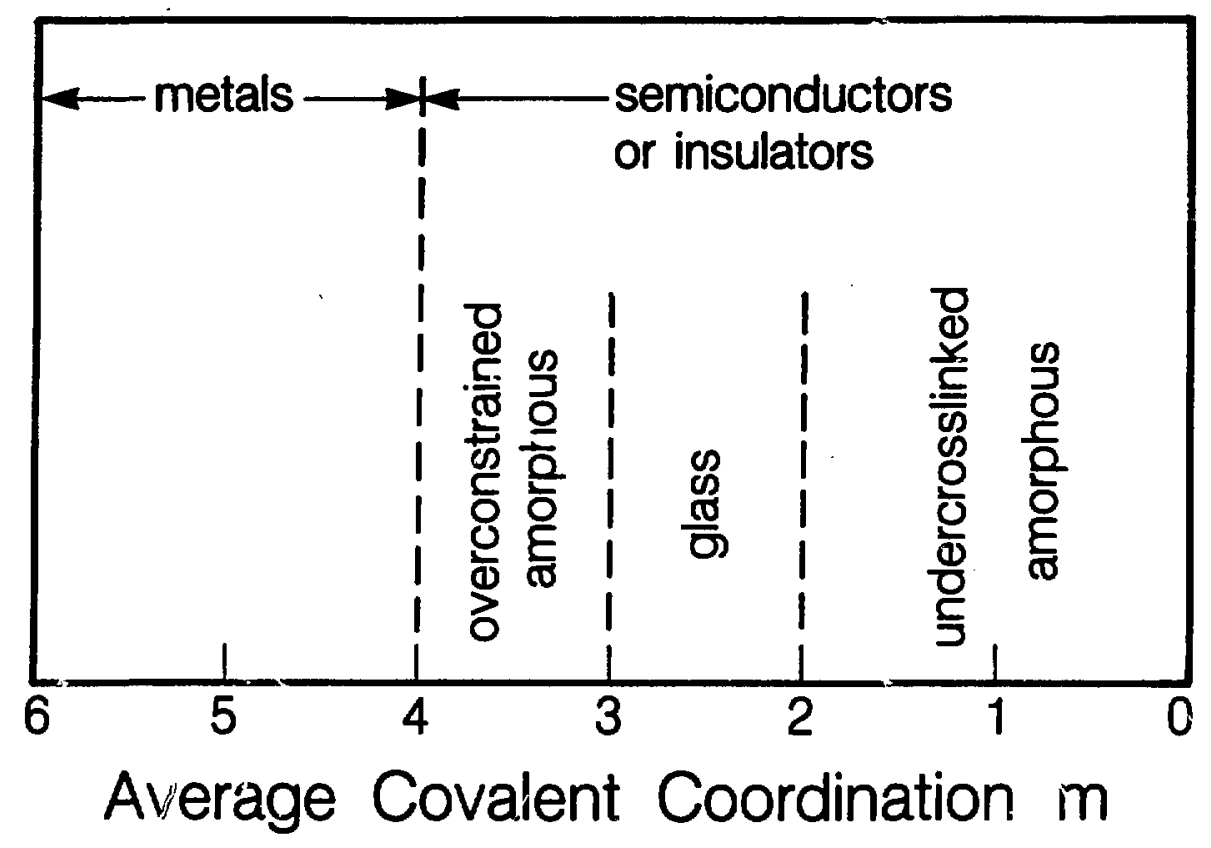

XBL 85iO-11729

Fig. 9. Classification of non-crystalline solids. 


\subsection{Amorphization Models}

Many authors have proposed amorphization models for ion implanted silicon, however each of them has been incomplete. The important ideas from these models will be discussed here in three categories: heterogeneous nucleation, homogeneous nucleation and critical deposition energy criterion.

\subsubsection{Heterogeneous nucleation models.}

As mentioned in Chapter 1 , the heterogeneous nucleation model describes amorphization for heavy ions which produce a high enough point defect density at the end of an individual ion track so that an amorphous zone is formed spontaneousiy. Morehead and Crowder $^{26}$ proposed a cylinder shape-damaged zone along an heavy ion track in the target as shown in Fig. 10a. A thermal spike surrounding the ion track dissipates in perhaps $10^{-12} \mathrm{~s}$, leaving a highly disordered region of many broken bonds and displaced atoms. These displaced atoms reform bonds and change their positions to form the amorphous phase, in a time of the order of $10^{-9} \mathrm{~s}$. During this time some vacancies escape via thermal diffusion from the disordered core surrounding the ion track. The outer sheath whose width is designated $\delta R(T)$ due to vacancies diffusion in time $\tau$ at temperature $T$ becomes crystalline. So, the final stable radius of the amorphous region, is assumed to be $R_{0}-\delta R$, and hence the number required for them to overlap and form a continuous amorphous layer, $\left(R_{0}-\& R\right)^{-2}$, depends both on the nuclear stopping power $\mathrm{dE} / \mathrm{dX}$, and the temperaturedependent out diffusion of vacancies which are assumed to reduce the 


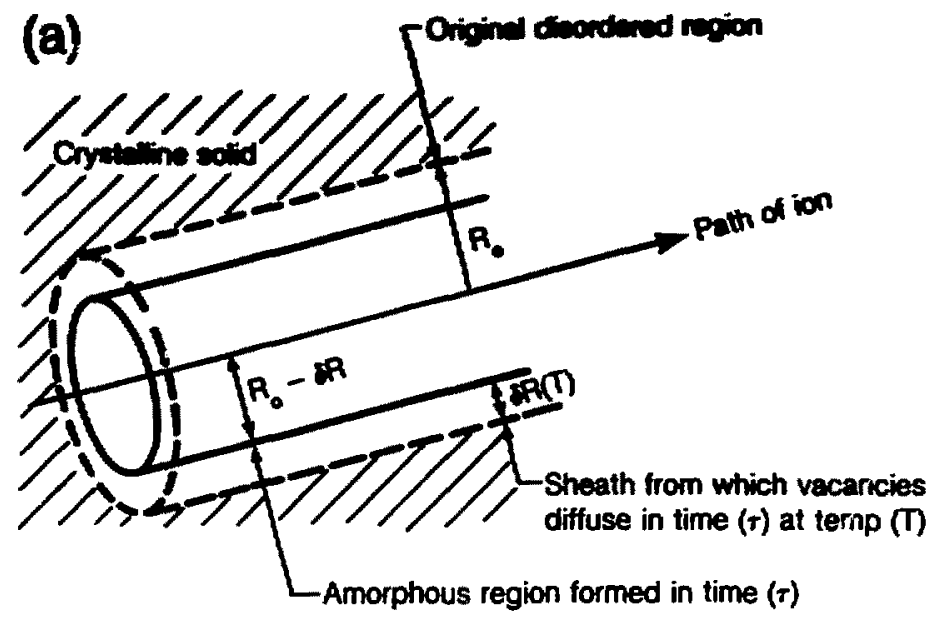

(b)

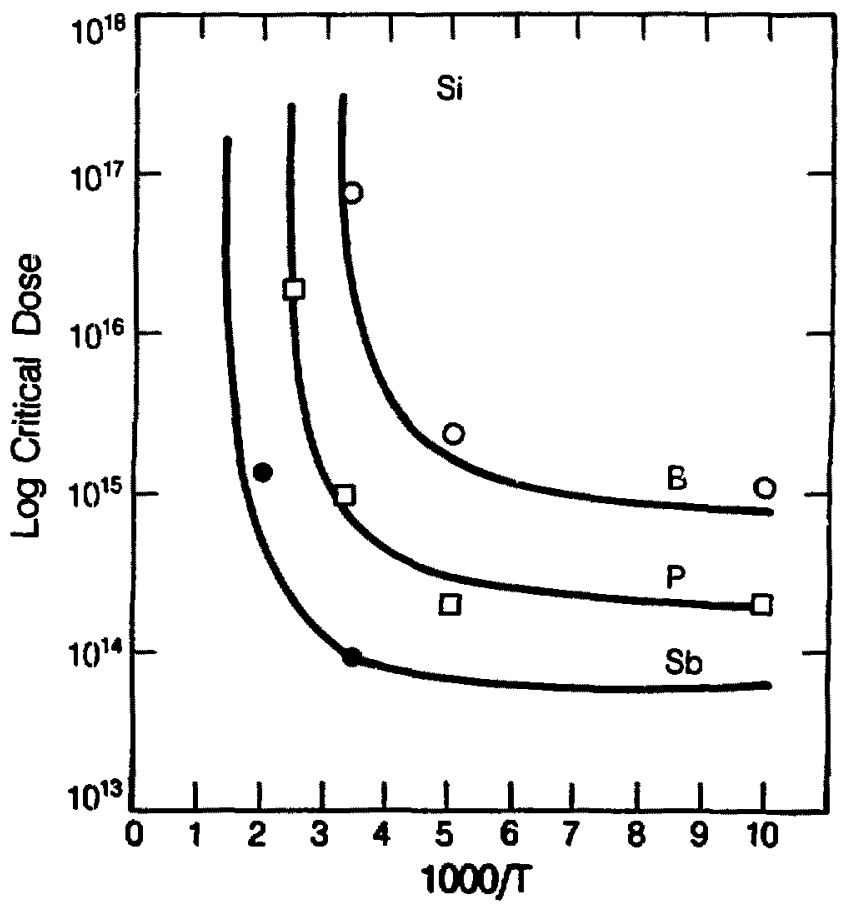

$x 640510-11720$

Fig. 10. (a) The damaged region surrounding the path of a high energy ion in a crystalline solid ideal ized as a cyl inder (dotted lines). Yacancies escape from the outer sheath and only the inner core (solida lines) becomes amorohous.

(b) A plot of $\log$ D vs. 1/T from equation in page 36 of text. The experimental points are taken from EPR data. 
size an anount oR. The critical dose for a amorphous layer is represented by:

$$
\phi(T)=\left(R_{0}-\delta R\right)^{-2}=\phi_{0}\left(1-\delta R / R_{0}\right)^{-2}
$$

where $\phi_{0}=R_{0}^{-2}=\bar{E} n_{2}(d E / d X)_{0}^{-1} \mathrm{~cm}^{-2}, \bar{E}$ is the effective energy to aisplace a target lattice atom in $e V, n_{2}$ is the number of target atom $\mathrm{cm}^{-3}$, and $(\mathrm{dE} / \mathrm{dX})_{0}$ is the nuclear energy loss per unit path length. The shrinkage of the cylinder can be estimated by the out diffusion of vacancies in the form of:

$$
\delta R=2\left(D_{v} \tau\right)^{1 / 2}
$$

where $D_{v} \simeq D_{v o} \exp \left(-E_{d f} / k T\right), D_{v}$ is the vacancy diffusion coefficient, $E_{d f}$ is the activation energy for the diffusion. The critical dose formulas can be expressed as a temperature dependent function:

$$
\phi(T)=\phi_{0}\left[1-K^{\prime}(d E / d X)_{0}^{-1 / 2} \exp (-U / K T)\right]^{-2}
$$

where $\quad K^{\prime}=2\left(D_{\text {vo }} \bar{E} n_{2}\right)^{1 / 2}$

and

$$
u=E_{d f} / 2
$$

By fitting available experimental data from backscattering measurements for continuous axorphous formation, ${ }^{66} \mathrm{~K}$ ' and $U$ were assumed to be $115(\mathrm{KeV} / \mu \mathrm{m})^{-1 / 2}$ and $0.06 \mathrm{eV}$. Using those fitted data, the results 
of EPR measurement for $B, P$ and $S b$ ions implanted silicon at various temperatures agreed qual itatively with the model, 67 as shown in Fig. $10 b$.

Although this simple model showed agreement with the experimental critical dose for amorphization at various temperatures, a contradiction exists in the $B$ ion implantation case, because a light ion such as $B$ ion cannot produce an amorphous zone near the end of an individual $B$ ion track. This contradiction casts some doubt as to the true interpretation of the two arbitrary constants $K$ ' and $U$. First, it should be recognized that backscattering measurements cannot distinguish between a continuous amorphous layer and a partially amorphous layer with highly deformed crystalline regions. For example, the backscattering from a silicon crystal tilted $9^{\circ}$ from the $\langle 100\rangle$ axis is the same as that from an amorphous layer. ${ }^{68}$ The determination that a layer is completely amorphous by EPR is also questionable.

Secondly, it is almost certainly a gross oversimplification to consider only motion of vacancies. The mobility of silicon self interstitials is much greater than that of vacancies at room temperature. Silicon interstitials are likely to play as important a role as vacancies in the radiation damage process that leads to the formation of armorphous material. Temperature dependence of the amorphous zone size could be attributed to the recombination of vacancies and interstitials in the region surrounding the ion track. The data fitting showed $U \approx E_{d f} / 2=0.06 \mathrm{eV}$. That suggests the activation energy for diffusion is equal to $0.12 \mathrm{eV}$ wich is far below the activation energy for annealing oi neutral vacancies $-0.33 \mathrm{eV} .69$ 
A modification of Morehead and Crowder's amorphization model was proposed by Gibbons. The formation of an amorphous region by light ions was assumed to involve the $n$ times overlapping of disorder zones produced by light ion damage. It was represented as

$$
A_{A}=A_{0}\left[1-\left(\sum_{k=0}^{k=n} \frac{\left(A_{j} \phi\right)^{k}}{k^{\prime}} e^{-A_{j} \phi}\right)\right]
$$

where $A_{A}, A_{0}, A_{j}$ and $D$ are total surface area covered by amorphous 'regions, the total area being implanted, projected area of each cluster on the surface, and the ion dose respectively. ${ }^{27,70}$ Agair, a good curve fitting can be obtained by choosing appropriate values of $A_{j}$ and $n$. This model did not mention point defeci interaction or diffusion during light ion implantation. The quantitative fitting was less convincing for ion implantation at temperatures higher than $4^{\circ} \mathrm{K}$.

Following Gibbon's ideas, a composite model for amorphization was proposed by Dennis and Hale. ${ }^{71}$ They used the deposition energy as a criterion for the amorphous zone formation by overlapping several disorder regions. The critical energy density for amorphization at liquid nitrogen temperature using ESR experimental data was found to be $6 \times 10^{23} \mathrm{eV} / \mathrm{cm}^{3}$. At high temperature, the out-diffusion of energy was considered to reduce the energy density in the damaged region. Subsequently, Hebb and Cater ${ }^{72-74}$ extended Gibbon's and Dennis Hale's models to subdivide the disordered but not amorphous region $A_{d}$ into separate regions $z_{1}, a_{2}, \ldots a_{m}$ which have different levels of 
stepwise accumulated damaged disorder. The results of numerical calculations of this and Gibbon's and Dennis and Hale's models were compared. One of the main differences between the composite (Dennis and Hale) and the overlap (Gibbon) models is that three different disorder areas were considered in the composite model. Each ion is assumed to produce, firstly an area $a_{a}$ which is directly amorphized, secondly an area $a_{d}$ which is damaged but not amorphized and thirdly, an area, $a_{c}$ (less than $a_{d}$ ) which, when overlapped with already damaged but not amorphized material, results in transformation of this region to amorphousness. The composite model can be expressed as follows:

$$
\begin{aligned}
\frac{A_{A}}{A_{0}}= & \left.1-\frac{a_{d}}{\left(a_{a}+a_{d}-a_{c}\right.}\right)\left\{\exp \left(-a c^{\phi)}\right\}-\frac{1}{a_{a}+a_{d}}\left\{a_{a}-\frac{a_{c}{ }^{a} d}{\left(a_{a}+a^{-a} c\right.}\right)\right\} \\
& \exp \left(-\left(a_{a}+a_{d}\right) \phi\right)
\end{aligned}
$$

In the composite model the amorphous-ion fluence function, plotted logarithmically, is always of sigmoidal form with a zero dose slope of unity; however, in the overlap amorphization models, the amorphousnessfluence function exhibits no turning points (i.e., non-sigmoidal) but: the zero fluence slope is greater than unity and dependent upon the over lap number, as shown in Fig. 11. Hebb and Carter's multiple divisions model is basically similar to the composite model in the amorphousness-fluence function. The major contribution from Webb and Carter is the consideration of annealing effects during ion inplantation which explained the dose rate effects on the amorphization. ${ }^{74}$ 


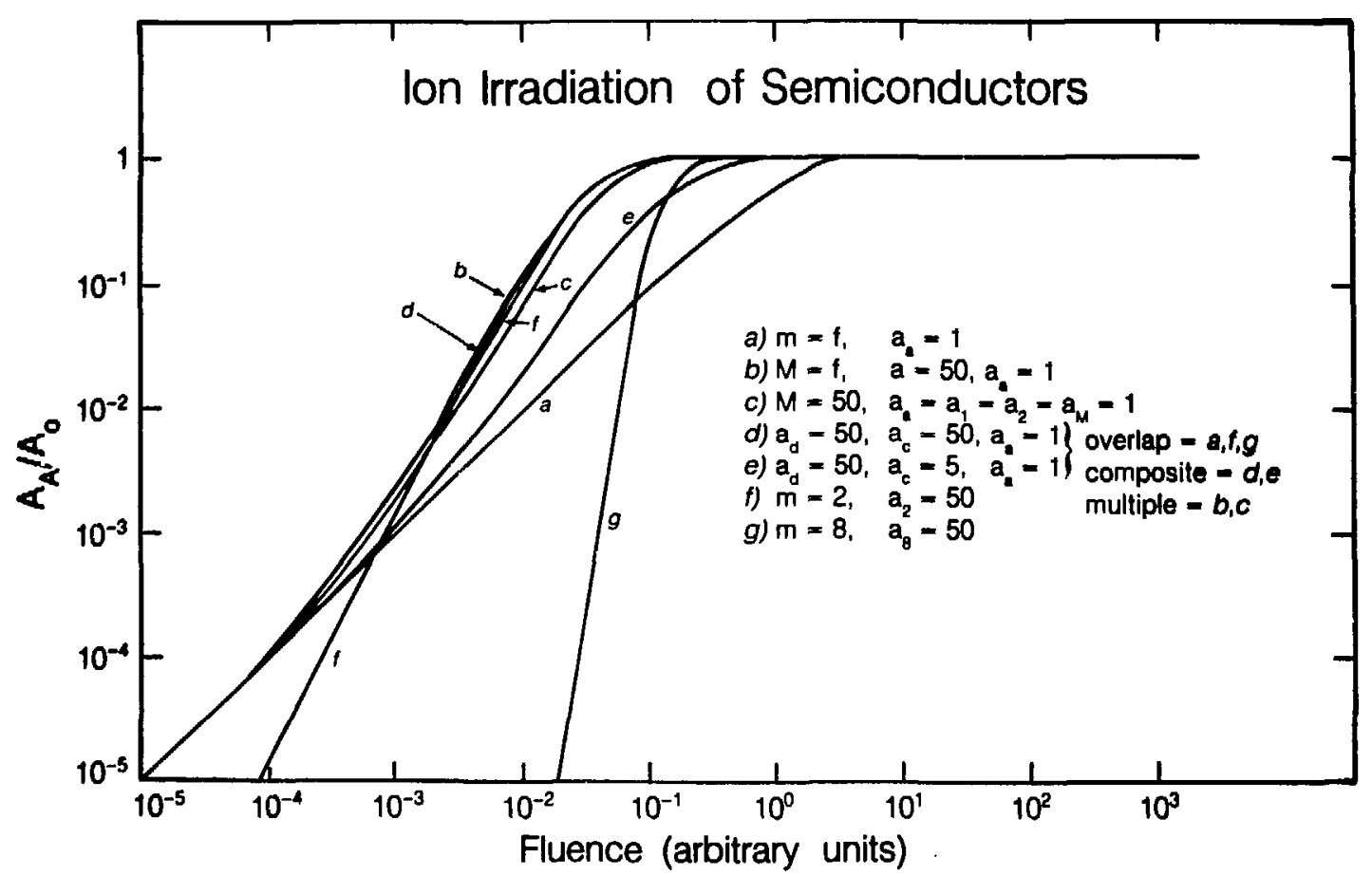

XBL 8510-11730

Fig. 11. Comparison of the different models for accumulation of amorphousness as a function of irradiated fluence. 
Although the composite model and the multiple division model show a two stage characteristics (i.e., sigmoidal form), the slope of unity at low dose is contradictory to the experimentally determined amorphousness-fluence function for light ion damage which is shown in Fig. 4 .

\subsubsection{Homogeneous nucleation models}

Vook and Stein discovered two prominent isochronal annealing stages above room temperature in neutron irradiated or ion damaged $\mathrm{Si}$ and $G e,{ }^{75}$ with the evidence from $x$-ray, electron microscope, infrared and electrical measurements. From a comparison of the time and temperature dependences of the annealing of electron, neutron, and ion damage in $\mathrm{Si}$, the lower temperature isochronal annealing stage near $200^{\circ} \mathrm{C}$ was considered as a consequence of the annealing of divacancies, of which the activation energy in motion and reorientation is $\sim 1.2 \mathrm{eV}$. The determination that the annealing of divacancies was being measured was supported by the appearance of the characteristic $1.8 \mu$ divacancy band in the infrared absorption spectrum. ${ }^{76}$ The high tenperature stage was shown to be near the temperature observed for the epitaxial growth of $\mathrm{Si}$ on a $\mathrm{Si}$ crystal substrate. It was interpreted as the annealing of amorphous $\mathrm{Si}$. By considering the anneal ing occurring during ion implantation, Yook and Stein concluded that "divacancy controlled annealing is responsible for the dose rate dependence of the crystalline to amorphous transition." This was the first discussion which connected the migration of point defects and amorphization by light ion damage. Furthermore, Yook and Stein observed very few 
divacancies immediately after $85^{\circ} \mathrm{K}$ implants of 400 KeV B ions, but during annealing growth of divacancies occurs between 150 and $300^{\circ} \mathrm{K}$ yielding a dersity almost equal to that for the same ion fluence at $300^{\circ} \mathrm{K} .77$ These results strongly suggested that below $300^{\circ} \mathrm{K}$ neutral vacancy motion (activation energy $\sim 0.33 \mathrm{eV}$ ) and trapping control the divacancy formation upon annealing for $B$ implants. The idea that homogeneous nucleation, which involves the accumulation of point defects can induce the crystalline to amorphous transition, was suggested by the discovery of point defect accumulation followed by subsequent disappearance as ion fluence increases. 78,79 is

Swanson et al.$^{79}$ suggested that the critical defect concentration at which a damaged region might spontaneously transform to a stable or metastable amorphous region can be estimated by assuming that it occurs when the free energy of the amorphous region equals that of the defectrich damaged region. 0.02 atomic fraction of defects in Si was estimated as the concentration required to transform the region into an amorphous volume, under the influence of the athermal atomic vibration. Thus, the homogeneous nucleation model for amorphization generally assumed that when the critical concentration of the accumulated point defects was reached, the damaged regions would suddenly transform to the amorphous state.

Chadderton also contributed an important concept of nucleation of damage centers during ion implantation of silicon. ${ }^{80}$ Homogeneous nucleation of interstitial clusters was thought to occur at low ion fluence and this is characterized, for an extremely short period of 
time, by a linear dependence of the state of disorder on ion fluence, followed by a long period during which disorder is proportional to (fluence) $^{1 / 2}$. During these periods of "nucleation" and "primary growth" small interstitial clusters behave as unsaturable traps. The disorder mentioned here was measured by the Rutherford backscattering channeling technique which detects the scattering of the aligned beam from atoms which are not located on lattice sites. Two primary assumptions of Chadderton's model are: 1) a constant homogeneous concentration of interstitials is generated in unit time during implantation; and 2) interstitials can interact with a homogeneous distribution of vacant lattice sites, nucleation traps, saturable traps and unsaturable traps.

A saturable trap is defined to be able to accept only one interstitial, after which it is no longer an effective site. Likewise, an unsaturable trap can accept migrating interstitials without limit, and without changing its subsequent capture cross-section. And a nucleation trap increases its capture cross-section with trapping of each interstitial. If $\mathrm{Ns}, \mathrm{Nu}$ and $\mathrm{Nn}$ represent respectively the number per unit volume of interstitials trapped at a fluence $\Phi=(\phi t)$ at saturable, unsaturable and nucleation sites, and $N s, N u$ and $N n$ are the numbers of such sites, the appropriate differential equations for fluence dependence at steady state are: 


$$
\begin{aligned}
& \frac{d n_{s}}{d}=\frac{v N_{s}(\Phi)}{D}=\frac{v\left(n_{s}(0)-n_{s}\right)}{D} \\
& \frac{d n_{u}}{d}=\frac{v N_{u}(\Phi)}{D}=\frac{v N_{u}(0)}{D} \\
& \frac{d n_{n}}{d}=\frac{v\left(N_{n}(0)+\gamma n_{n}\right)}{D} \\
& \frac{d n_{v}}{d}=v\left(1-\frac{n_{v}}{D}\right)
\end{aligned}
$$

with

$$
D=N_{s}(\Phi)+N_{u}(0)+n_{v}(\Phi)+\left(N_{n}(0)+r n_{n}(\Phi)\right)
$$

where $\left(N_{n}(0)+\gamma n_{n}\right)$ is the effective number per unit volume of nucleation sites at any time $t$ if $N_{n}(0)$ is the number at $t=0, r$ is an arbitrary parameter, $n_{v}(\Phi)$ is the instantaneous vacancy concentration, and $v$ the effective number of defects per ion.

With the approximation of the same scattering probability for various interstitial clusters and with the same number of vacancies and interstitials at the beginning, the mean additional number of scattering events per particle can be given by:

$$
m(\Phi)=n_{v}(\Phi) \bar{m}
$$

where $\bar{m}$ is a mean scattering probability.

If the unsaturable traps are quite dominant, the influence of other $k$ inds of trap is negligible, so that

$$
\left.m(0)=n_{u}(0)\left(\frac{2 v}{n_{u}(0)}+1\right)^{1 / 2}-1\right\}=
$$


which for high $\Phi\left(2 v \Phi \gg N_{u}(0)\right)$, becomes

$$
m(\Phi)=\left(2 u N_{u}(0) \Phi\right)^{1 / 2} \text { 再 }
$$

Without specifying the nature of unsaturable traps, Chadderton successfully demonstrates that a (fluence) ${ }^{1 / 2}$ dependence of implantation induced disorder by light ions in silicon is a characteristic of nucleation at traps.

Figure 12 is a schematic representation of the dependence of implantation disorder as a function of the fluence $\Phi$ of light ions, showing the effects anticipated for changes in flux $(b)$, mass $\left(M_{1}\right)$ temperature $(T)$ and prior doping with impurities, following Chadderton's simple point defect clustering model. The disorder between $A$ and $B$ points in Fig. 12 is a function of (fluence) $)^{1 / 2}$ dependence. Between $B$ and $C$, however, the secondary growth is characterized by linear fluence dependence. It can be explained by assuming that nucleation traps are dominant. By simple integration of the above differential equation for nucleation traps:

$$
n_{n}(\phi) \frac{(1+\gamma)}{r}-\frac{n_{n}(0)}{\gamma^{2}} \log _{e}\left(1+\frac{r n_{n}(\phi)}{n_{n}(0)}\right)=v \Phi
$$

wich for high $\left(n_{n}(\phi) \gg n_{n}(0)\right)$ becomes:

$$
n_{n}(0)=n_{v}(0)=\frac{y(u)}{(1+v)}
$$




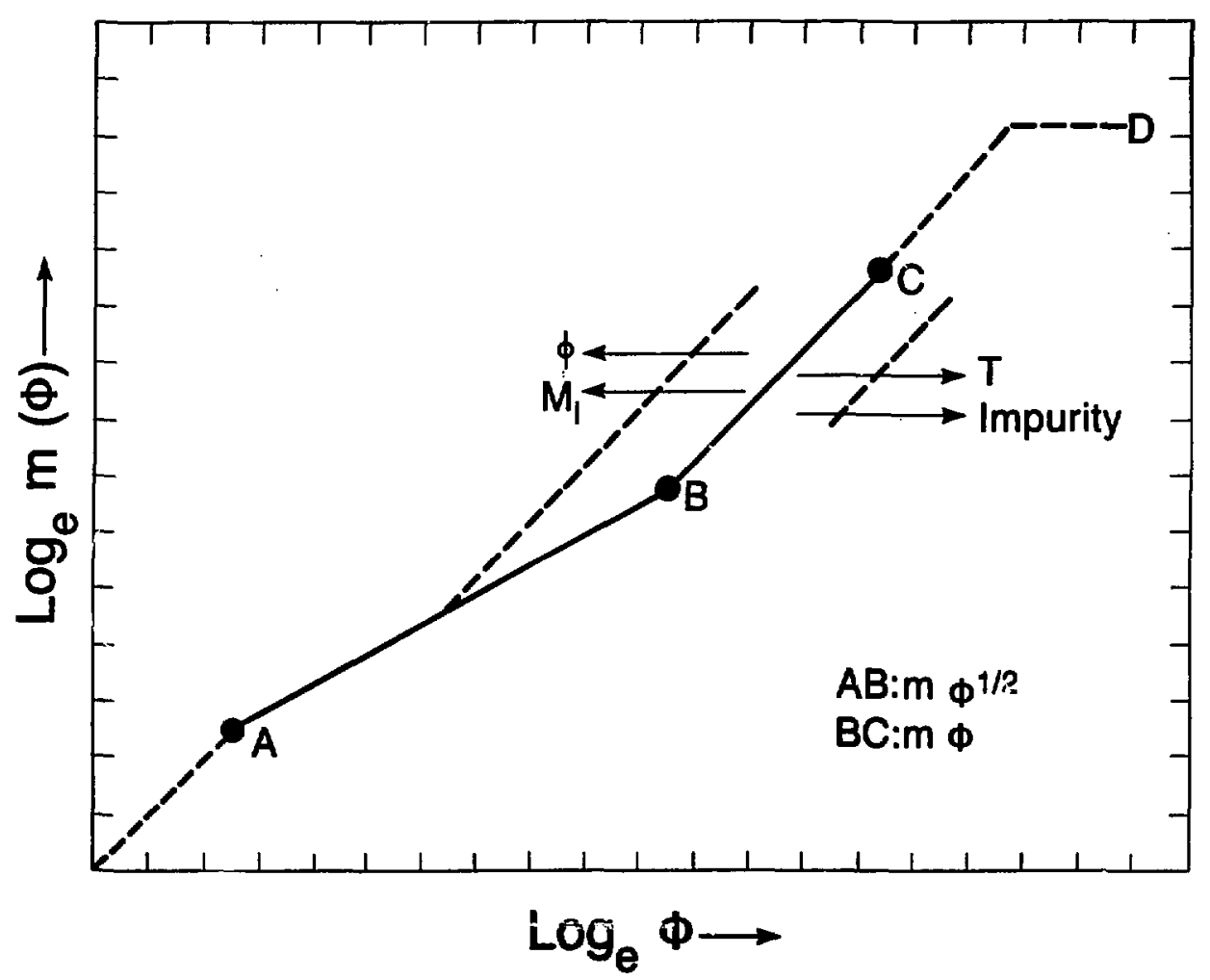

XBL 8511-11513

Fig. 12. Schematic representation of the dependence of implantation aisorder in silicon as a function of the fluence for light ions, showing the effects for change in flux (6), mass $\left(M_{1}\right)$, temperature $(T)$ and prior doping with impurities. 
For a higher fluence, between $C$ and $D$ in Fig. 12, the disorder saturates at the time when nucleation traps become saturable traps and these become dominant. So the disorder parameter is expressed:

$$
m(\Phi)=N_{s}(0)\left\{1-\exp \left(-\frac{v}{N_{s}(0)}\right)\right\}_{m}
$$

which for high $\Phi\left(\nu \Phi \gg N_{s}(0)\right)$ becomes:

$$
m(\Phi)=N_{s}(0) \bar{m}
$$

and is constant.

rhadderton's homogeneoue nucleation model gave a rough picture of the point defect trapping and homogeneous nucleation of point defect clusters, which explained the (fluence) $)^{1 / 2}$ dependence for RBS defined disorder. However, events occurring at the atomic level during the crystalline to amorphous transition between $C$ and $D$ (Fig. 12), which corresponds to the second stage in IR reflection results (Fig. 4), are still open to conjecture.

\subsubsection{Critical deposition energy criterion.}

A simple criterion for the formation of amorphous material by ion implantation, that does not require any specific view of the structure, has been proposed by Stein et al., 81 using the corzept that there is a critical energy deposition as nuclear stopping which is necessary to displace enough lattice atoms for morphization. An energy deposition per unit volume of $-10^{21} \mathrm{kew} / \mathrm{cm}^{3}$ was found to be the 


\section{critical energy for the formation of an amorphous layer at room tem-} perature. ${ }^{70}$ The energy deposition is defined as a product of nuclear stopping power and ion fluence, where nucleation stopping power is a function of ion penetration energy. The average nuclear energy loss rate at the position $x$ can be expressed:

$$
\left\langle S_{n}(x)\right\rangle=\int_{0}^{\infty} S_{n}(E) p\left(E x, E_{0}\right) d E
$$

where pdE denotes the probability that an ion has energy between $E$ and $E+d E$, given that it was injected into a target at $E=E_{0}$ and has reached a position $x$ along its track, $S_{n}(E)$ is Thomas-Fermi nuclear stopping power which was discussed by LSS. ${ }^{20}\left\langle S_{n}(x)\right\rangle$ function, when plotted versus $x$, is also known as the energy deposition profile or damage profile. Both Brice ${ }^{82}$ and Gibbons ${ }^{83}$ made contributions to the theoretical prediction of the energy deposition profile. Figure 13 shows an example of the damage profile of $B^{11}$ ion implantation in silicon with $40,100,200,300,400 \mathrm{KeV}$ respectively, which are the results of Brice's calculations. Many other authors also determined the damage profiles due to ion implantation by various experimental measurements. ${ }^{84-87}$

If the critical energy deposition of $\sim 10^{21} \mathrm{KeV} / \mathrm{cm}^{3}$ is correct for various ions implantation in silicon at room temperature, a $1 \times 10^{16} / \mathrm{cal}^{2}$ boron ion implant into silicon with $100 \mathrm{KeV}$ at room temperature should produce a continuous amorphous layer from surface to about $3900 \AA$ depth, as derived from the damage profile in Fig. 13. 


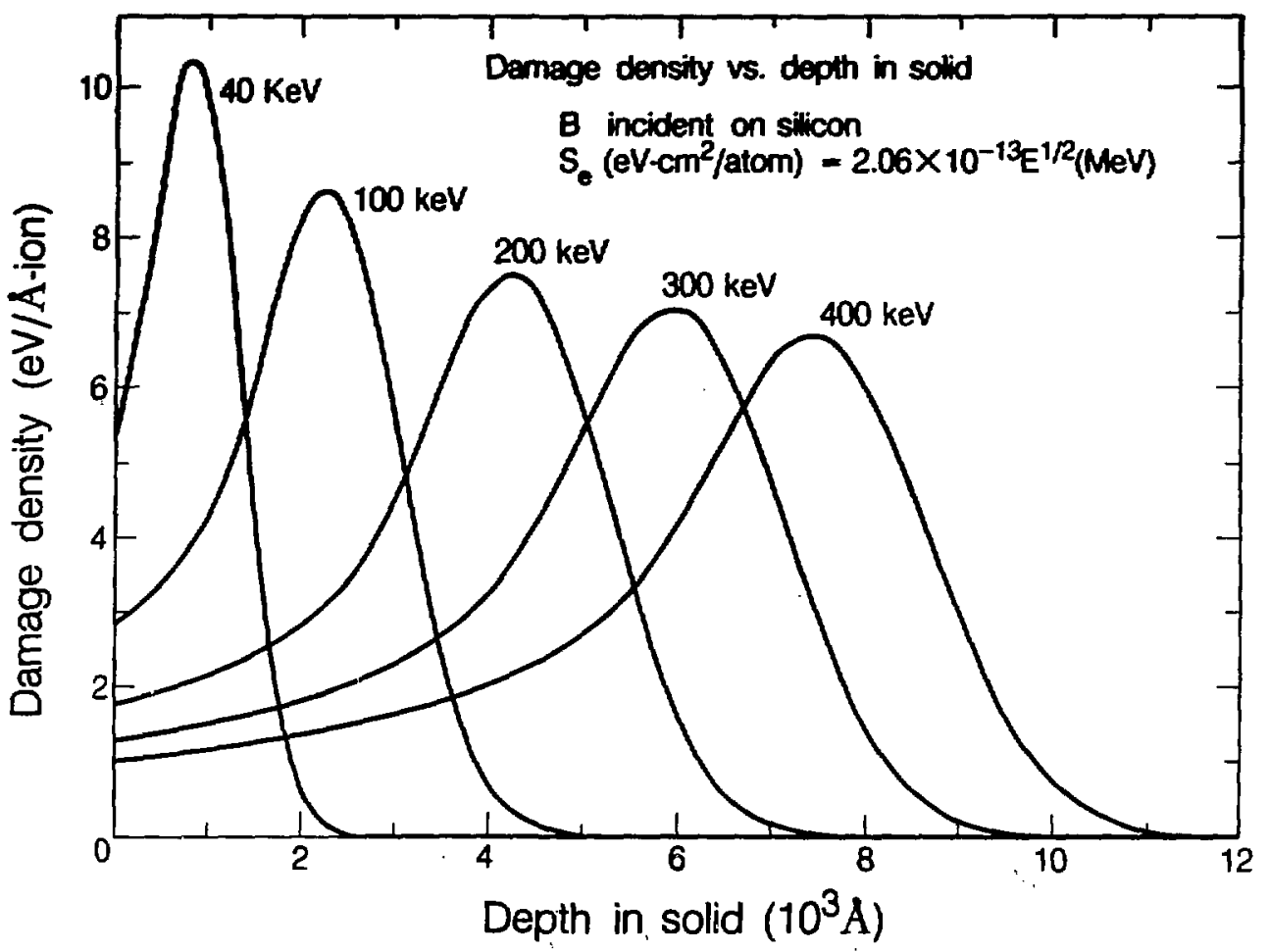

XBL 854-8844

Fig. 13. Depth Jistribution of deposition energy density for boron ion implantation on silicon. 
However, no anorphous layer can be found in such a specimen, wich shows that the criterion is not universally applicable. This is due to the fact that the $10^{21} \mathrm{KeV} / \mathrm{cm}^{2}$ critical number was calculated using the crude standard nuclear stopping power and the amorphization critical dose from ESR data (shown in Fig. 10.b). In fact, the critical energy deposition varies with implantation temperature, dose rate, ion mass and accelerating voltage as shown by recent experimental results, especially TEM cross section measurements which is the most accurate way to determine the position of the amorphous-crystalline interface ${ }^{88,89}$ It is not difficult to understand the reasons for the breakdown of this criterion. Amorphization by ion implantation can be through different mechanisms, heterogeneous and homogeneous nucleation or others, when different ions, temperatures, and dose rate are used. Later, the out diffusion of silicon interstitials will be discussed as the factor which is responsible for the increase of the critical energy deposition at lower accelerating voltage.

However, the critical energy deposition criterion gives a rough idea for predicting the position of the crystaitine-amorphous interface especially at low temperatures, at which the damage produced by a projectile will be retained as stable damage. This criterion can be improved if the diffusion of point defects is taken into account. 


\section{PROPOSED AMORPHIZATION RECHANISM FOR LIGHT ION DAMUGE}

\section{A two stage characteristic of amorphization has been strongly} suggested by the experiment of IR reflection for light ion implantation at room temperature, especially for boron which is the lightest ion used in real I.C. fabrication processes. The initial stage of amorphization by light ion damage is considered as a homogeneous nucleation, during which damage clusters are formed through thermal diffusion and trapping of point defects produced by the ion radiation damage. Although the detailed process of point defect interaction is not fully understood, the idea of point defect cluster formation and accumulation is generally accepted. The simple point defects, vacancies and self interstitials, which have high mobility and are unstable at room temperature, ${ }^{69,77}$ have to recombine or aggregate into larger point defect clusters which are essentially immobile and thereby are stable at room temperature.

The second stage of amorphization, which is a gradual and progressive process, has hardly been investigated. The nature of this stage holds the key to understanding amorphization by light ion damage. Based on previous theoretical and experimental results, an amorphization mechanism for light ion damage is proposed. An amorphous zone is proposed to be formed at the end of an incoming light ion track, when the critical concentration of the preaccumulated point defect clusters is built up to a critical value at this region.

\subsection{Two Possible Amorphization Mechanisws}

Depending on the uniformity of point defect cluster distribution, 
two possibilities for the second stage of arorphization are suggested for light ion implantation at room teaperature. 90

A. Point defect cluster accumulation followed by heterogeneous formation of amorphous zone.

The first stage of amorphization is assumed to be the accumulation of uniformly distributed point defect clusters and complexes, which should be primarily the smallest size of point defect clusters being stable at room temperature, such as divacancies $V-B, V-C, V-0$ complexes, diinterstitials, and 4 vacancies, etc. After the concentration of these point defect clusters reaches a critical value, it is assumed that at the end of even a light ion track there is enough additional damage to form a new amorphous zone.

B. Amorphous phase nucleation and growth.

During the accumulation of point defects clusters in the first stage, a very few small amorphous zones may be formed due to the overlapping of several ion tracks within a short period of time. These amorphous zones are assumed to act as nuclei for growth of larger amorphous regions. The total volume of these amorphous nuclei is at first extremely small so that no noticeable increase of refractive index would be observed in the first stage. Because of the lesser density of the amorphous state compared with that of the crystalline state, there would be compressive strain fields around the amorphous nuclei. Vacancies would tend to diffuse toward and accumulate around the nuclei to reduce this strain energy. Higher concentration of point oefect clusters and complexes could therefore be formed near 
already existing amorphous nuclei than elsewhere. The amorphous nuclei would grow whenever an additional ion track ended within a region of high enough cefect density near the already existing amorphous regions. The rate of formation of amorphous material would be expected to accelerate as the size of the amorphous zones increased until amorphous zones begin to impinge on one another leading to an s shaped amorphous volume fraction vs. fluence curve.

The two stages of amorphization for these two proposed mechanisms and the concentration of point defect clusters as a function of distance away from an existing amorphous nuclei are schematically shown in Fig. 14. The number of displaced atoms at the end of each implanted boron ion track can ce estimated with a few assumptions. When the boron ion energy has been reduced to $3 \mathrm{KeV}$ at which nuclear stopping dominates the energy loss, the remaining ion path length is $250 \AA$ according to the LSS theory. ${ }^{21}$ If the radius of the disordered zone and the atomic displacement energy are assumed to be $10 \AA$ and $15 \mathrm{eV}$, the atomic fraction of lattice displacement in the disordered zone $(\sim \pi \times 100 \times 250 \AA)$ is calculated as $2.5 \%$. Cristel, Gibbons and Sigmond suggested that displacement of about $10 \%$ of the lattice at $77^{\circ} \mathrm{K}$ will cause amorphization. 24 vacancies and interstitials are less mobile at $77^{\circ} \mathrm{K}$, therefore a higher concentration of smaller defect complexes and elementary point defects would be expected as compared to room temperature implantation. The critical concentration for point defects and/or small clusters accumulated during the first stage of amorphization should be about $7.5 \%(10 \%-2.5 \%)$. Mechanisa A (Fig. 14) assumes 
Mecthanism (a)

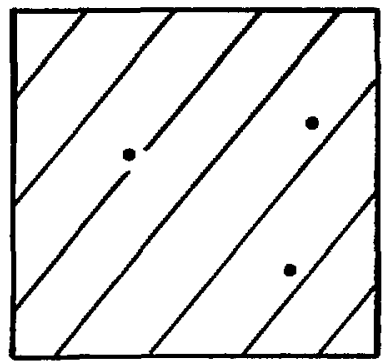

Accumulation of point defect cluster and complexes.

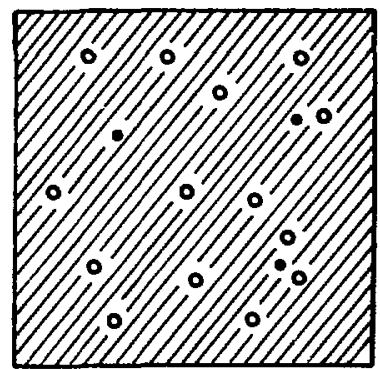

New amorphous zones formation.

\section{Stage 1}

Stage ॥

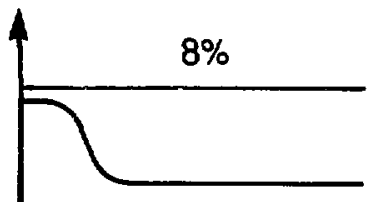

(a)

(b)

Nuclei interface

Conc. of lattice displacement

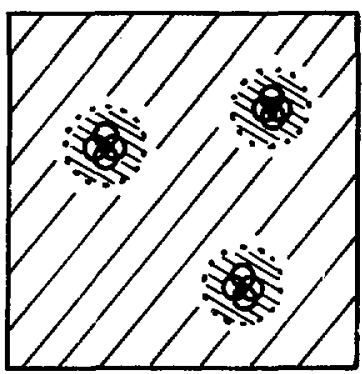

Growth of amorphous nuclei. (n)
Mectanism (b)

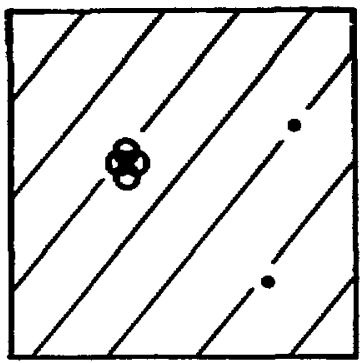

Amorphous nuclei formed by ion track overlapping. 
that a uniform distribution of preaccumulated point defects and/or clusters is maintained up to the critical concentration $(-8 \%)$. Mechanism $B$ assumes that the concentration of point defects increases preferentially near the amorphous nuclei and reaches the critical value earlier than elsewhere.

The uniformity of point defect distribution depends on two factors, the mobility of point defects and strain fields. If the scanning of the ion beam during implantation is assumed to be uniform, at a low temperature at which vacancies and interstitials are essentially frozen, the accumulation of the point defects would be expected to be uniform, thus mechanism $A$ should dominate the amorphization process. Uniformity of the accumulated point defects might be expected to decrease if the implantation temperature is raised to a "mobile" temperature. In this case vacancies and selfinterstitial are mobile and therefore preferential accumulation of point defects must be considered. Pure mechanism A might shift gradualiy toward mechanism B at nigher temperature, the separation of the first and second stages of amorphization would become less clear at higher temperature.

3.2 A Model of Amorphous Zone Formation by Light Ion Damage

3.2.1 The importance of interstitial type defects for amorphization.

The crystalline to amorphous transition occurs primarily during the second stage of amorphization when the point defects concentration has reacheo the critical value in all or a large fraction of the irradiatec volume. A few concepts concerning the amorphous structure 
which have been discussed in Chapter 2.1, have to be restated here. First, the density of amorphous silicon is about 1-2\% less than that of crystalline silicon, either from the calculation of an ideal random network amorphous structure, 32 or from the $x$-ray measurement for the latice expansion during the microcrystalline to amorphous transition in the experiment of plasma thin film deposition. ${ }^{45}$ This small change in density suggests that point defects clusters of both vacancy and self interstitial types are involved during the accumulation in the first stage of amorphization. The transformation from crystalline to amorphous results in a volume "expansion" of the transformed region in the crystalline matrix, rather than a "collapse" of vacancy enriched regiuns. "Collapse" of the crystalline lattice has often been used as a description in the previous amorphization models. $14,26,78,79$ The volume expansion induces lateral stress in silicon which have been measured. ${ }^{91}$ It is suggested in this model that the concentration of vacancy type point defects should not be used as the only parameter of importance in the amorphization process. Silicon interstitials which have the possibility of athermal migration ${ }^{69}$ under ionizing radiation diffuse faster than vacancies in silicon at low temperature. Therefore some silicon interstitials probably escape from the irradiated volume either to the surface or into the interior. This is less true for vacancies. Although ion damage produces Frenkel pairs with the same number of vacancies and self interstitials, the concentration of interstitial defects would be expected to be less than that of vacancy aefects after ion implantation. Therefore, a critical concentration of interstitials and/ar small interstitial clusters may be 
an important parameter for amorphous zone formation, probably at least as important as an accumulation of vacancies.

In Chapter 2.1, it was pointed out that during the crystal to amorphous transformation five to eight fold rings replace the "chair" type six fold rings of the diamond cubic lattice. The loss of 3 rd nearest neighbors in amorphous structure which was shown in $x$-ray scattering spectrum, is the result of the replacement of six fold rings during the crystalline to amorphous state. The five to eight fold rings model of the amorphous structure will be useful to understand the transition from crystalline state containing point defects to the amorphous state.

\subsubsection{Point defect clusterings.}

Owing to the high diffusivities of vacancies and interstitials at room temperature (estimated to be $D_{1}=3.16 \times 10^{-4} \mathrm{~cm}^{2} / \mathrm{s}, D_{v}=$ $\left.4.15 \times 10^{-9} \mathrm{~cm}^{2} / \mathrm{s}\right), 92$ they would recombine, aggregate or react with other impurities. The possible reactions of point defects are expressed as follows:

$$
\begin{aligned}
& v+I \rightarrow d \text { (recombination), } \\
& v+v \rightarrow v_{2} \\
& v_{2}+I \rightarrow v \\
& I+I \rightarrow I_{2} \\
& v+0_{I} \rightarrow v_{0} \\
& v 0+I \rightarrow 0_{I} \\
& I+c_{s} \rightarrow c_{I} \\
& C_{I}+v \rightarrow C_{s}
\end{aligned}
$$




$$
\begin{aligned}
& \mathrm{VO}+\mathrm{C}_{\mathrm{I}} \rightarrow \mathrm{C}_{\mathrm{S}} \mathrm{O}_{\mathrm{I}} \\
& \mathrm{B}_{\mathrm{S}}+\mathrm{I} \rightarrow \mathrm{B}_{\mathrm{I}} \\
& \mathrm{B}_{\mathrm{I}}+\mathrm{I} \rightarrow \mathrm{B}_{\mathrm{I}} \mathrm{I}
\end{aligned}
$$

where $V$, and I stand for vacancy and silicon self interstitial; Vo is vacancy-oxygen complex ( $A$ center). $C_{S}$ and $B_{S}$ are substitutional carbon and boron impurities which can trap Si self-interstitials to transfer to interstitial impurities. 93,94

Amorphization by ion implantation can occur in low oxygen and carbon content silicon and it also can occur by silicon ion self implantation. Hence, the impurity effects on point defect accumulation, al though it is important in specific cases, is excluded here for the consideration of amorphization by 1 ight ion damage. The most important reactions which contain only vacancies and interstitial, could be as follows:

$$
\begin{aligned}
& V+I \rightarrow D \text { (recombination) } \\
& V+V \rightarrow V_{2} \\
& V_{2}+I \rightarrow V \\
& V_{n}+V \rightarrow V_{n+1} \\
& I+I \rightarrow I_{2} \\
& I_{2}+V \rightarrow I \\
& I_{n}+I \rightarrow I_{n+1}
\end{aligned}
$$

Although the charge state effects of point defect are neglected for simplicity here, they could also be important. 95 The annealing properties of interstitial related defects and vacancy-related defects in silicon are shom in Fig. 15 (a) and (b).96 These defects which were 

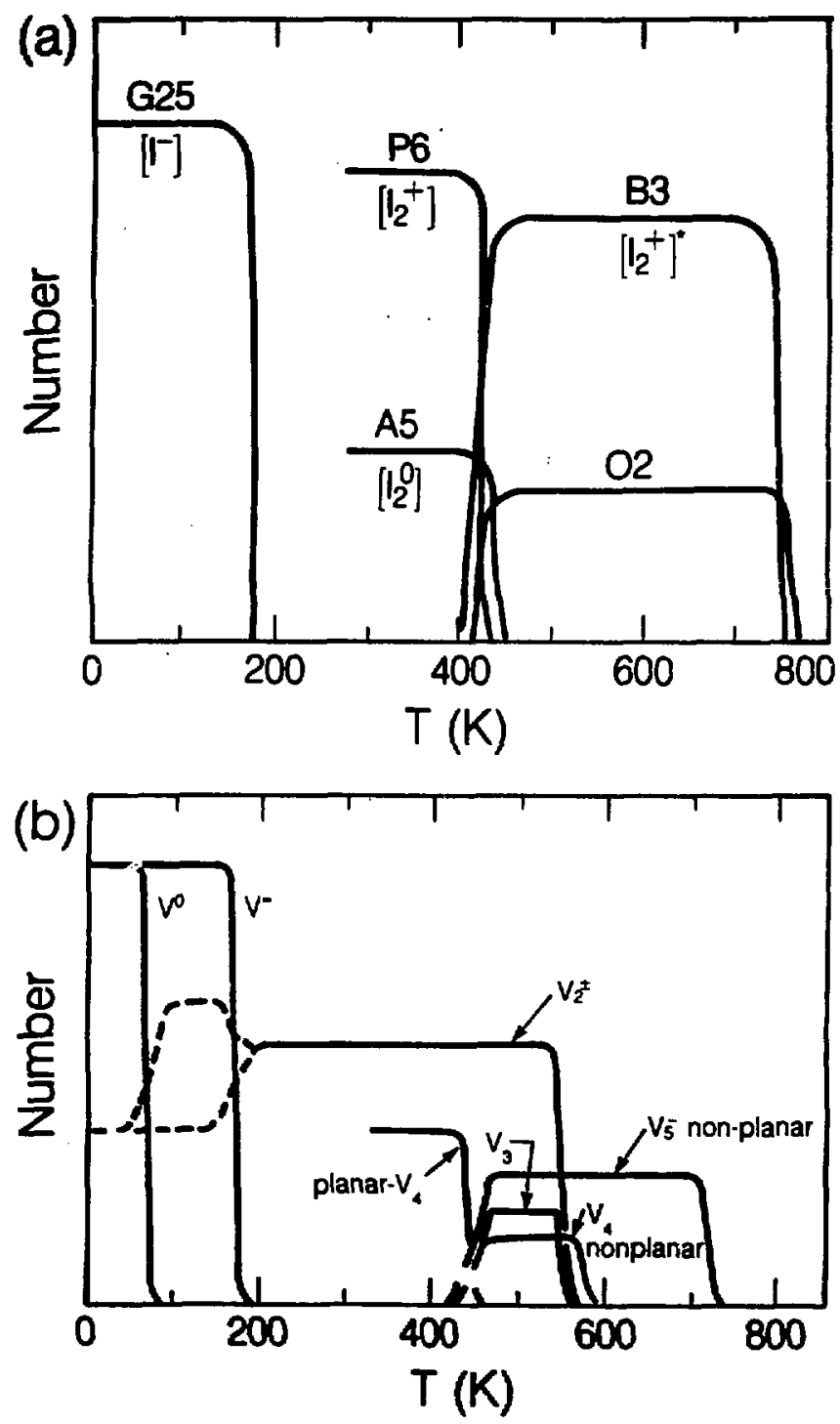

XBL $8510-11720$

Fig. 15. Schematic :epresentation of the recovery of several EPR spectre attributed to (a) interstitial defects in silicon; (b) for intrinsic vacancy-related defects in silicon. 
detected by EPR showed that simple vacancies and interstitials vanish except below $200^{\circ} \mathrm{K}$. The major point defects which are stable at $300^{\circ} \mathrm{K}$ room temperature are diinterstitials and divacancies, al though planar 4 vacancy clusters are also found around room temperature. The proposed diinterstitial configuration for the $\mathrm{P} 6$ center defect in Fig. 15 (a) is shown in Fig. 16(a) with a split in $<100\rangle$ directions. 97,98 Another possible diinterstitial configuration in which the two interstitials aggregate along $\langle 110\rangle$ axes is also shown in Fig. 16(b)..$^{99}$ The aivacancy configuration has been modeled as Fig. 17 by Watkins and Corbett in 1965.100 The activation energies for annealing of the radiation induced point defects are summarized in Table 2, after Corbett and Watkins, 101 and Seeger and Frank. 102

In addition, the migration energy of the P6 defect, the $\langle 100\rangle-s p l i t$ diinterstitial was estimated to be $0.6( \pm 0.1)$ eV. 98 The activation energy for reorientation of $\langle 110\rangle-\mathrm{I}_{2}^{+}$and $\langle 100\rangle-\mathrm{I}_{2}^{0}$ which were shown in Table 2, were suggested to be 0.70 and $0.92 \mathrm{eV}$ by internal friction measurements. 69

\subsubsection{Embryo of an amorphous zone; the pairing of divacancies} and diinterstitials.

The major accumulated point defects, which are stable during room temperature implantation, are divancies and diinterstitials. They can only recombine through the dissociation of divacancies and/or diinterstitials into single vacancies and/or interstitials. Havever, the dissociation of a divacancy or diinterstitial is improbable at room temperature because the increase in free energy is large compared to kT. 
(a)
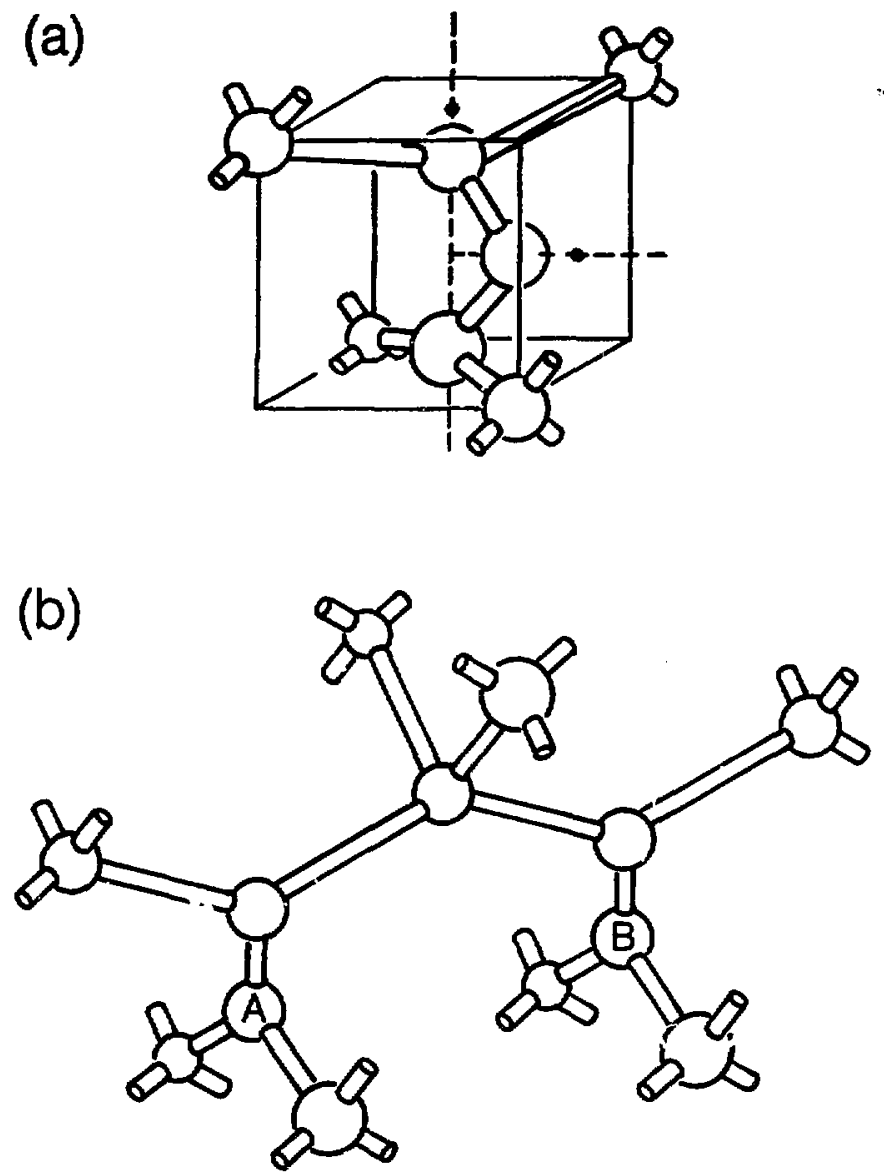

XBL 8510-11721

Fig. 16. (a) Defect model of the $\langle 100\rangle-s p l i t$ di interstitial in a distorted configuration; (b) diinterstitial along a <110> chain in diamond cubic structure. 


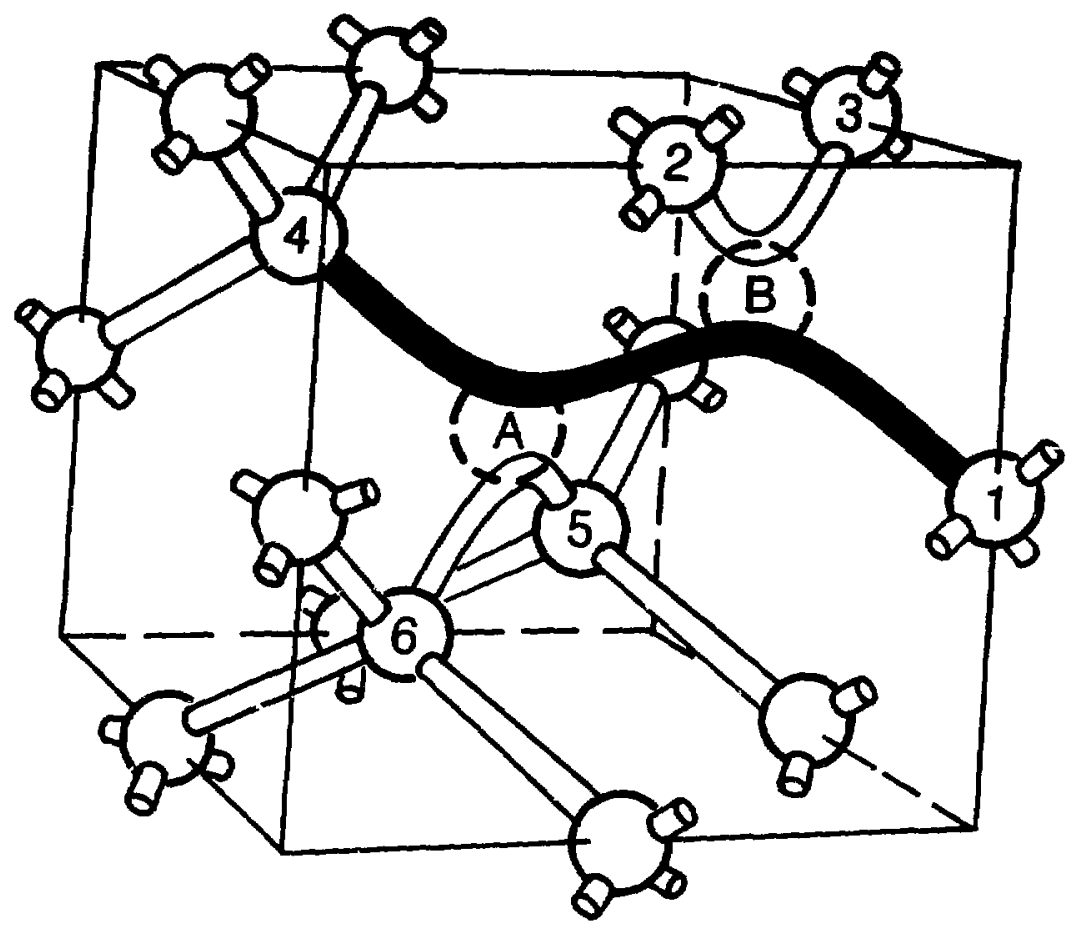

XBL 8510-11719

Fig. 17. The divacancy in the diamond lattice. 
Table 2.

Amealing properties of radiation-induced point defects in silicon. Y and I stand for vacancy- and interstitialtype defects, respectively. Upper indices indicate the electrical charge state, lower indices the number of single defects contained in a cluster.

\begin{tabular}{cccc}
\hline $\begin{array}{c}\text { Annealing } \\
\text { Temperature } \\
{[\mathrm{k}]}\end{array}$ & $\begin{array}{c}\text { Anneal ing } \\
\text { Defect }\end{array}$ & $\begin{array}{c}\text { Activation Energy } \\
\text { for Anneal ing } \\
{[\mathrm{eV}]}\end{array}$ & Doping \\
\hline$<4$ & $\mathrm{I}($ athermal) & -0.0 & $\mathrm{P}$ \\
$70-80$ & $\mathrm{v}^{-2}$ & 0.18 & $\mathrm{n}$ \\
140 & $\mathrm{I}^{-}$ & -0.4 & $\mathrm{n}$ \\
$150-180$ & $v^{0}$ & 0.33 & $\mathrm{P}$ \\
$370-420$ & $<110>-I_{2}^{+}$ & 0.85 & $\mathrm{P}$ \\
570 & $<100>-I_{2}^{0}$ & 1.5 & $\mathrm{n,P}$ \\
$570-670$ & $\mathrm{~V}_{2}$ & 1.5 & $\mathrm{n,P}$ \\
\hline
\end{tabular}

Considering the atomic rearrangement during the crystalline to amorphous transition, of a small region containing a divacancydiinterstitial pair there would be very little change in volume. It is suggested that such a pair constitutes an embryo of the amorphous phase. The bonding structure surrounding a divacancy (as shown in Fig. 17) on a layer of the (110) plane in silicon can be described as shown in Fig. 18(a). The six-fold rings are replaced by 2 five fold and 1 eight fold ring at the center of the divacancy. Aiter the 


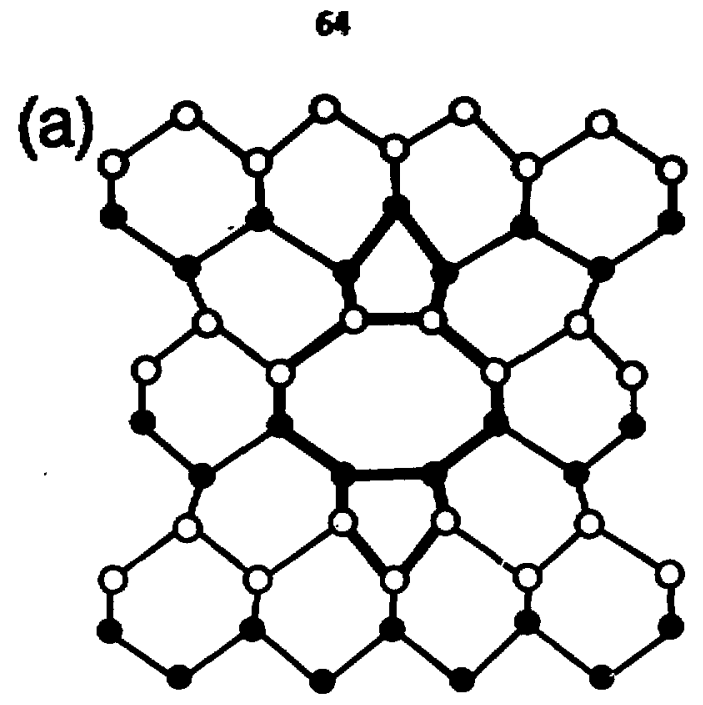

(b)

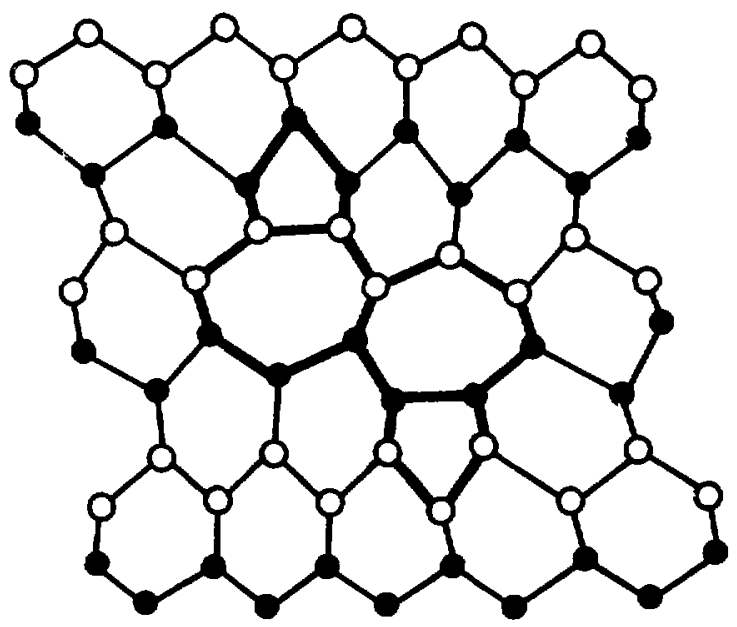

XBL 8510-11723

Fig. 18. (a) The bonding (projected onto the (110) plane) surrounding a divacancy along a $\langle 110\rangle$ chain; (b) after rebonding of (a). 
lacalized rebonding of the structure in fig. $18(a), 2$ five fold rings and 2 seven fold rings are formed as show in Fig. 18(b). The bonding surrounding a split <110> diinterstitial on a layer of the (110) plane can be cescribed as in Fig. 19(a). Again 2 five fold rings and 2 seven fold rings are formed (Fig. $19(\mathrm{~b})$ ) at the center of the diinterstitial after a small rebonding of the structure in Fig. 19(a).

The structure of amorphous silicon is five to eight fold rings. Therefore, if the two centers, a divacancy and a diinterstitial with the rebonded structures get close enough, the region has a structure that is very similar to a small region of amorphous silicon. Small rearrangements may permit its transformation to the amorphous structure. The energy required for breaking silicon covalent bonds to initiate the rebonding of dinterstitials and divacancies is about the order of one electron volt. The strain energy of the point defects or the thermal vibration energy of the silicon lattice is far below the energy needed for rebonding. Only the nuclear stopping energy brought by an incoming ion can break silicon bonding and reform five or seven fold rings in the region near the ion track. Therefore amorphous zones should be formed near the end of light ion tracks when the light ion enters a region with a critical concentration of the accumulated divacancies and di interstitials at room temperature. Four vacancy clusters and two diinterstitials could also play a similar role to that of divacancy-diinterstitial pairs as an embryo of an amorphous 2one. However, the most probable case would be the divacancy diinterstitial pair which constitute the majority of the accunulated point defect clusters. 
(a)

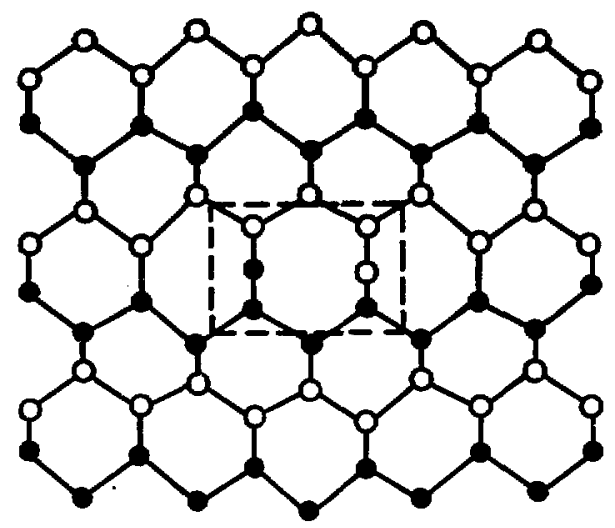

(b)

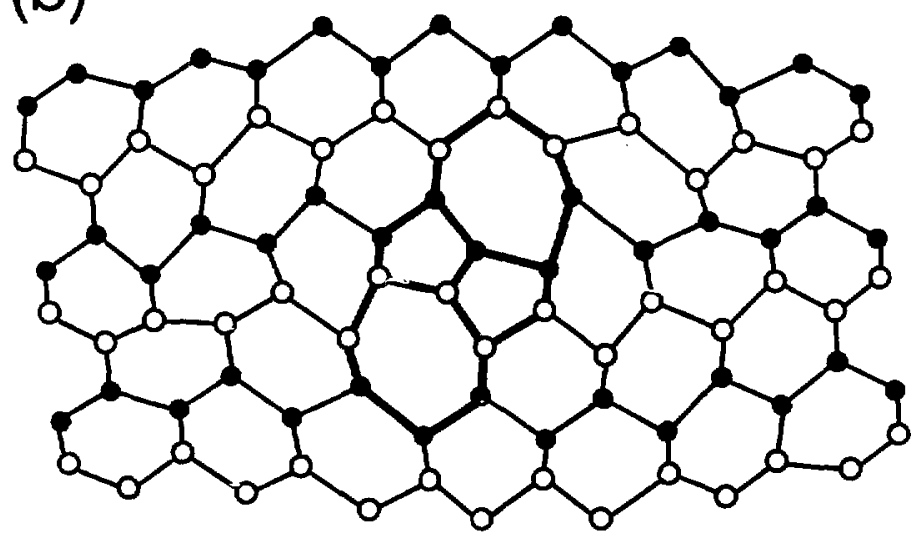

XBL $8510-11722$

Fig. 19. (a) The bonding surrounding a split-<110> diinterstitial. (b) Rebonding of the structure in (a). 


\section{EXPERIRENTAL BASIS FOR AMORPHIZATION MECHAMISH}

\subsection{Experimental Approach}

4.1.1 High resolution TEM observation at amorphous-crystalline transition regions in a cross-section specimen.

The cross section view of the amorphous-crystalline transition region affords a continuous picture of the amorphous to crystalline transformation process, where the amorphous material is first formed at the damage peak then expands in both directions as the implanted dose is increasea (Fig. 20). Lattice image high resolution TEM observations at the transition region can clearly identify the amorphous zones and the crystalline regions. In addition, near amorphous regions large interstitial clusters and small interstitial or vacancy loops can be seen under high resolution TEM.

\subsubsection{EPR (Electron Paramagnetic Resonance) detection of} amorphous zone formation and point defect clusters.

EPR can detect and identify small amorphous zones and small point defect clusters which are too small for TEM observation by their specific magnetic resonance spectrums. The density of the dangling bonds in amorphous regions and the concentration of point defect clusters can be determined by the density of spin at the specific $g$ values during the amorphization process. Small amorphous zores $(-20 \AA)$ buried in a matrix of silicon crystalline material would be very difficult to detect using high resolution lattice imaging TEM, convergent beam TEM or microdiffraction by TEM. EPR affords strong evidence of the presence of amorphous zones by the appearance of an isotropic resonance 


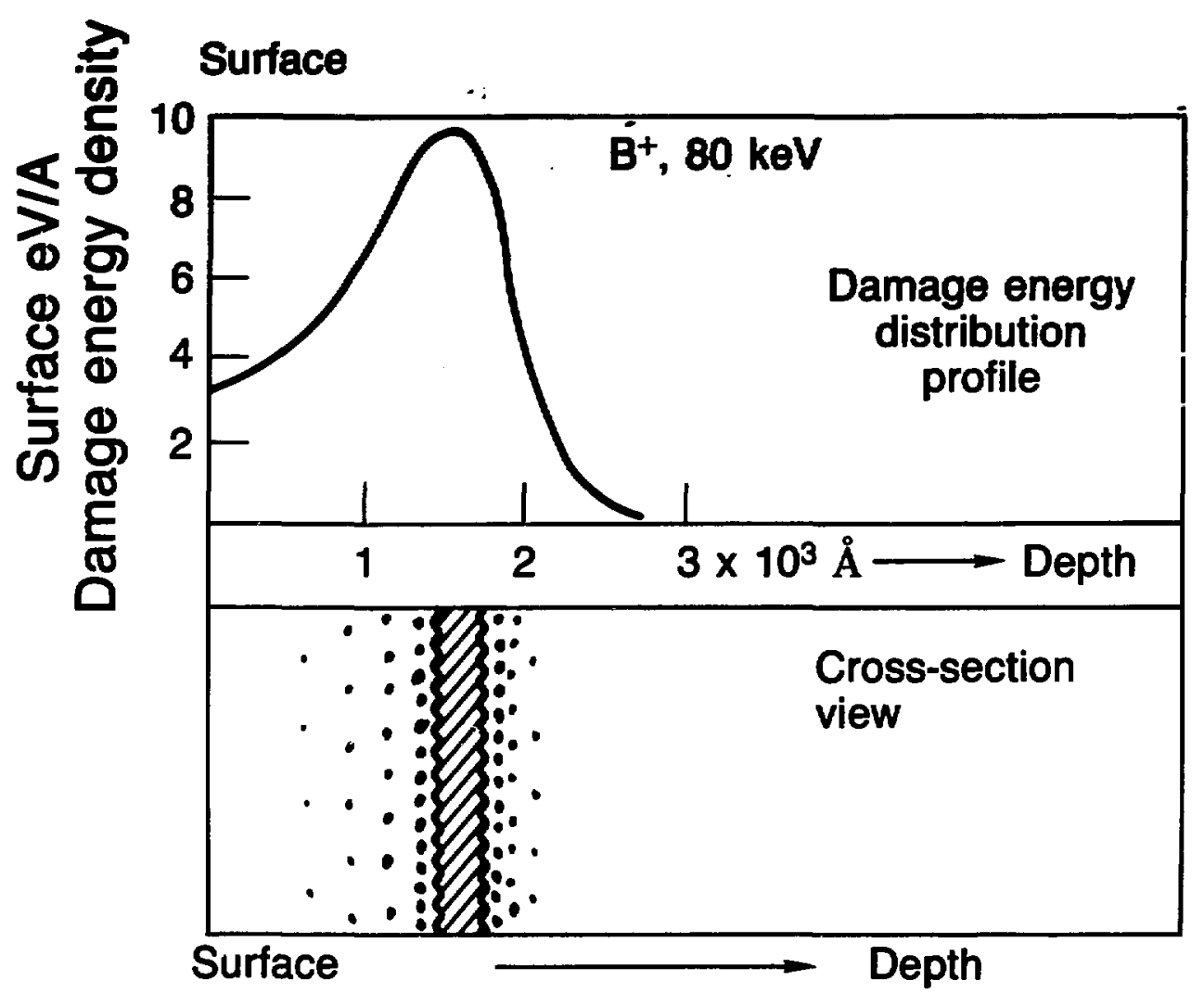

XBL 854-11023

Fig. 20. Schematic illustration of cross-section view by ion damage and damage energy distribution profile. 
related to the dangling bonds. For small complexes of point defects only anisotropic peaks are found. $44,48-50,103,104$

\subsubsection{The effects of dose rate (beam current) and implantation temperature on amorphization.}

At a temperature where elementary point defects are mob:le, high dose rate shortens the diffusion time for point defects before the next near-by event produces additional point defects. The probability of formation of small complexes such as divacancies and dinterstitials is increased and out diffusion of elementary defects is reduced. Low temperature implantation $\left(<200^{\circ} \mathrm{K}\right)$ has a similar effect to that of high dose rate through lowering of the mobility of point defects. With an increase of implantation temperature, the recombination rate and the out diffusion of point defect are increased.

4.1.4 High voltage ion implantation.

Very high energy ( MeV) ion implantation has been tried to achieve vertical device structure. Some novel and important device structure have been fabricated using ion beams with energy between 1 and $5 \mathrm{MeV} .{ }^{105-107}$ The topic of the damage induced through megavolt ion implantation into silicon is not only interesting, but also crucial to the aevice structure. The wide amorphous-crystalline transition region $(-2 \mu)$ in $11 \mathrm{MeV}$ arsenic ion implanted silicon results in the formation of tangled dislocation networks after recrystallization at $545^{\circ} \mathrm{C}$ and annealing at $965^{\circ} \mathrm{C} .108$ A buried amorphous layer which is far away from the bombarded surface, is formed by megavolt ion implantation, if the ion dose is enough for amorphization. In this case fewer mobile point defects are lost by migration to the surface. 


\subsection{Experimental Procedure}

\subsubsection{The starting materials and ion implantation.}

Silicon wafers, $\langle 100\rangle$ or iented and boron doped P- type, having 5 $10 \mathrm{~cm}$ resistivity were obtained from a commercial supplier. Boron ions with fluences of $1 \times 10^{15} \sim 3 \times 10^{16} / \mathrm{cm}^{2}$ were implanted in silicon wafers which were attached to an alumina plate holder with silver paste to ensure good thermal conductivity. The implantation energy was $80 \mathrm{KeV}$ for room temperature and is $100 \mathrm{KeV}$ for the liquid nitrogen temperature case. The beam current was $1 \sim 2 \mu \mathrm{A}$ to avoid beam heating effects during implantation. Silicon wafers were tilted $8^{\circ}$ off [100] orientation with the ion beam axis to prevent ion channelling. The beam size was 1-2 cm diameter with uniform scanning during implantation.

The high dose rate sample was ion implanted with $35 \mathrm{KeV}$ and $2 \mathrm{~mA}$ beam current at room temperature. Good thermal contact with the holder was required. to ensure that the silicon wafer did not exceed $50^{\circ} \mathrm{C}$. Temperature color incication tape was attached on the backside of the wafer, which showed that the wafer temperature reached only $35^{\circ} \mathrm{C}$. This high beam current implantation was performed with a boron ion fluence of $5 \times 10^{15} / \mathrm{cm}^{2}$ on a $\langle 111>$ silicon wafer.

The high energy boron ion implantations were carried out using the facilities of the Lawrence Berkeley Laboratory Hilac. The accelerator is a dynamitron with a 2.2 MeV terminal voltage. The silicon, substrate was either water cooled to a nominal $300^{\circ} \mathrm{K}$ or liquid nitrogen cooled to $77^{\circ} \mathrm{K}$. A dose of $1 \times 10^{15} / \mathrm{cm}^{2}$ boron ion was deposited with $4 \mathrm{MeV}$ for all the high energy implantation specimens. 
The double implantation specimens were implanted first with boron, and second with silicon. Another specimen was implanied with reverse sequence for comparison. The boron ion fluence of $5 \times 10^{15} / \mathrm{cm}^{2}$ and the silicon ion fluence of $5 \times 10^{14} / \mathrm{cm}^{2}$ were implanted at $80 \mathrm{keV}$ and $125 \mathrm{KeV}$, respectively at room temperature.

\subsubsection{Cross-section specimen preparation for Transmission} Electron Microscopy.

For TEM cross-section specimens, especially for ion implanted samples where surface layers of thickness of only a few $1000 \AA$ are to be viewed, the preparation method involves mechanical thinning followed by ion milling. ${ }^{109}$ The first stage for specimen preparation is to mechanically polish to a thickness of $25 \mu \mathrm{m}$, which is about the thickness through which dark-red light can be viewed. Two specimens of dimension $1 \mathrm{~cm} \times 1 \mathrm{~cm}$ were then glued together face to face with epoxy. The glued specimen was squeezed by a clamp during drying of the glue for one day in order to make the two face to face surfaces close enough so that the surfaces are not preferentially attacked during ion milling. Then the specinen was mounted on a glass or quartz disc with crystal bond with a silicon slice $1 \mathrm{~cm} \times 1 \mathrm{~cm}$ on either side for support. This is schematically illustrated in Fig. 21. The mounted specimen was polished flat with 240 grit SiC paper followed by another 600 grit SiC paper and $6 \mu m$ diamond paste. A final $1 \mu \mathrm{m}$ polish was sometime given to improve the surface finish. Then, the sample was turned over, renounted and the polishing sequence repeated to give a final specimen about 25 in thick. 


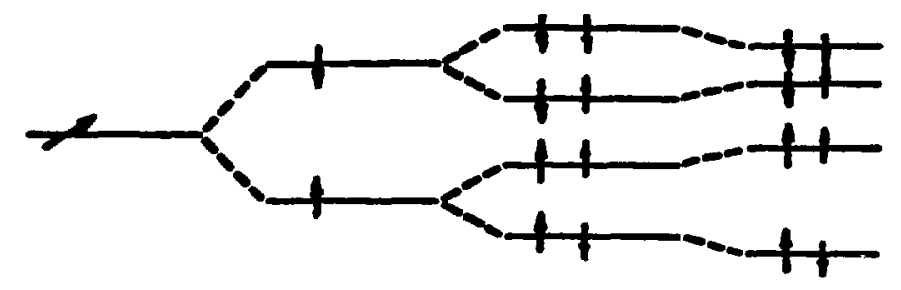
Non-magnetic
Zeeman
Hyperfine
Nuclear

$$
\begin{array}{cc}
\chi=\chi_{\mathrm{O}} & +g_{S} \beta \overrightarrow{\mathrm{H}} \cdot \overrightarrow{\mathrm{S}} \\
\Delta h \nu_{\mathrm{S}}= & g_{\mathrm{S}} \beta \mathrm{H}
\end{array}
$$$$
+A \vec{S} \cdot \vec{I}
$$$$
-g_{1} \beta \vec{H} \cdot \vec{\imath}
$$
$+A m_{1}$
$-g_{1} \beta \mathrm{Hm}$,

XBL 8511-12209

Fig. 21. Illustration of energy level splitting for a sample with $S=1 / 2, I=1 / 2$ spin system. 


\section{3}

The polished thin specimen was then mounted on a $3 \mathrm{~mm}$ copper grid. The final thinning was accomplished with argon ion milling at $12 \sim 15^{\circ}$ specimen tilt, $4 \sim 5 \mathrm{KeV}$ gun voltage, and $\sim 20 \mu \mathrm{A}$ specimen current. The rotating specimen stage of the Gatan ion mill was cooled with liquid nitrogen to avoid beam heating effects. A laser detector was used to control the termination of ion milling when a hole in the center of the specimen appeared. If the hole is through the line cf the glued surfaces, the specimen is ready for TEM observation.

\subsubsection{Lattice imaging high resólution Transmission Electron:} Microscopy.

Most of the TEM observation in this study was done in the lattice imaging mode, produced by the interference of many diffracted beams at a [110] symetrical zone axis. The resolution for lattice naging is limited by lens aberrations and microscope instabilities. The most serious limitation is imposed by the spherical aberration of the objective lens.

A beam $g$ is imaged as $\exp [i x(g)]$ by the lens, where $g$ is diffraction vector, $X$ is the phase and $\exp [i x(g)]$ is the contrast transfer function (CTF) of the lens. For axial illumination the phase $X$ of a beam emitted from the specimen at an angle a to the optical axis can be expressed 110

$$
x(a)=\frac{\pi}{\lambda} \frac{c_{s} a^{4}}{2}+D a^{2}
$$

where $\lambda$ is wavelength, $C_{s}$ is spherical aberration and $D$ is object ive 
defocus. The contrast in a high resolution lattice image is essentially phase contrast, and thus it is the imaginary part of the CTF. At maximum contrast, differentiating the above equation with respect to $g$ (where $\alpha=\lambda g$ ), the value of optimum defocus can be obtained as:

$$
D_{s c h}=1(2.5 / 2 \pi)\left(C_{s} \lambda\right)^{1 / 2}=-1.2\left(C_{x} \lambda\right)^{1 / 2}
$$

which is known as the Scharzer defocus. ${ }^{111}$

At Scherzer defocus the spherical aberration limits the point to point resolution to $d_{s c h}=0.7 c_{s}^{1 / 2} \lambda^{3 / 4}$.

In addition to the effect of objective spherical aberration as a limitation on resolution, several practical steps for taking high resolution micrograpli are necessary. They are 1) optimizaticn of the illumination condition, 2) optimum trade off between brightness and beam coherence for condenser aperture, 3) careful al ignment of the lens and beam axis, 4) the highest symmetry in the selected area diffraction pattern, and 5) the correct adjustment of objective astigmatism. The most difficult step in this procedure is the objective astigmatism adjustment. The astigmatism correction was usually done by the observation of amorphous material. At the correct astigmatism setting, the amorphous material should show isotropic fine-grain contrast as the focus is taken above and below the minimum contrast condition. (Theoretically, $D_{m c}=-0.44\left(c_{s} x\right)^{1 / 2}$ ) A thru-focal series of images, from over focused condition to the defocused condition beyond Scherzer defocus, was uswally taken at the desired area. 
Microscopes used for this study were the Simens 102 and the JEOL-JEM 200CX. The Weak Beam Dark Field inages and Bright field images were taken in Siemer 102 with a high angle tilting ability. The lattice images were taken in JEM $200 C X\left(C_{s} \simeq 1.22 \mathrm{~mm}, D_{m c} \simeq-240 \mathrm{~A}\right.$, $D_{\text {sch }}=-660$ A) with $\mathrm{LaB}_{6}$ filament at $200 \mathrm{KeV}$ voltage.

\subsubsection{EPR (Electron Paramagnetic Resonance) measurement.}

Para agnetic resonance is a form of spectroscopy in which an oscillating magnetic field induces magnetic dipole transitions between the energy levels of a system of paramagnetr. EPR is only concerned with the magnetic dipoie transition of electronic origin, especially unpaired electrons. The chemical bonding in a perfect semiconductor or insulator is usually such that the valence electrcns are spin paired according to the Pauli principle. Under these corditions the solid is diamagnetic and yields a null EPR spectrum. Imperfections such as vacancies, interstitials, impurities, and dangling bonds in solid often have localized state with energy levels within the bandgap. Frequently, one or several of the defect's charge states exhibit an unpaired electron and thus paramagnetism. The effects of temperature, light illumination, $n-$ or p-type doping, etc., can change charge state and thus induce or quench the EPR signal in existing defects. 112

The relative splittings of the localized defect level containing an unfaired electron can be described by a spin Hamiltonian of the rather general form: 113 


$$
H_{\text {spin }}=\text { HB }_{\mathrm{S}} \cdot \vec{g} \cdot \vec{B}+\overrightarrow{\mathrm{S}} \cdot \overrightarrow{\mathrm{D}} \cdot \overrightarrow{\mathrm{S}}+\sum_{\mathbf{j}} \overrightarrow{\mathrm{S}} \cdot \vec{A}_{\mathbf{j}} \cdot \vec{I}_{\mathbf{j}}
$$

(electronic (spin-spin) (hyperfine) Zeeman)

$$
\begin{aligned}
& -\sum_{j} \frac{g_{n_{i} j^{{ } W}}}{I_{j}} \overrightarrow{I_{j}} \cdot \vec{B}+\underset{j}{\Sigma_{I_{j}}} \cdot \overrightarrow{Q_{j}} \cdot \overrightarrow{I_{j}} \\
& \text { (nuclear Zeeman) (quadrupole) }
\end{aligned}
$$

The various magnetic interactions are represented by explicit terms in the spin Hamiltonian; whereas, the spatial parts of the wave functions reflecting Coulomb interaction, exchange, symmetry, etc., are buried in the $\overleftrightarrow{3}, \overleftrightarrow{D}, \overleftrightarrow{A}, \overleftrightarrow{Q}$. etc. coupling tensors. The $\overleftrightarrow{g}$ tensor gives the overall symmetry of a defect. The numerical values for $g_{k \ell}$ are usually unique to each defect. Very minor perturbations (charge state, neighboring impurities, etc.) tend to affect it to a measurible extent. The specific $g$ value works as a fingerprint for defects in thr EPR spectrum. It makes EPR handy and powerful for identificatior of defects. The $\overleftrightarrow{A}$ represents the hyperfine interaction, which is the magnetic dipole-dipole interaction between the unpaired electrons $(S)$ and nuclei with nuclear spin I. The spin-spin interaction which are represented by the $\overleftrightarrow{D}$ tensor, occurs for defects and impurities with $S \geq 1$. The quadrupole interaction (Q tensor) may only occur for nuclei with $I>1 / 2$ and is due to the interaction between electron quadrupole moment of a nucleus and electrical field gradient of the crystal at the nucleus. This interaction is usually not perceptible in EPR measuremint, but may need to be considered in electron-nuclear-double-resonance (ENDOR) experiments. 114 
Typical energy level splittings for a simple $S=1 / 2, I=1 / 2$ spin system are illustrated in Fig. 21 (e.9.. $\mathrm{Si}^{29}$, wich is -5 abundent in nature, of $H^{1}$ ).

Considering the example of a dangling bond on a three-fold coordinated silicon atom, if the magnetic field $H_{0}$ is parallel to the $\mathrm{Sp}^{3}$ dangling bond, the $g$ : alue $\left(g_{\sharp}\right)$ can be show by symmetry arguments to be unshifted (to first order) to $g_{e}$ (for a free electron $g_{e}=2.0023$ ). If $H_{0}$ is perpendicular to the dangling bond, the g-value is shifted to $g_{1}$. In an amorphous system, these defects will have random orientations with respect to $H_{0}$. The resultant line spans the range from $g_{\|}$to $g_{\perp}$ with highest density near $g_{1}$ $\left(g_{1} \simeq 2.0080\right) .^{115}$ The EPR line shape in undoped amorphous silicon is very nearly independent of deposition conditions. It was initially seen in evaporated $S i$ and guessed to be due to dangling bonds. ${ }^{44}$ The featureless 1 ine at $g=2.0055$ has also been observed in sputtered Si (with or without hydrogen), glow discharge and chenically vapordeposited Si, crystalline Si self-implanted to amorphicity, etc. ${ }^{48-50,103,104}$ The line broadening of amorphous silicon with the variation in the g-tensor could be due to bond angle and length fluctuation in amorphous materials. The hyperfine interaction could also produce unresolved line broadening due to the overlapping of a neighboring nuclear spin or from a much-reduced splitting.

ER 200D-SRC EPR facility from IBM Instruments, Inc., (manufactured by Bruker) was used in this study. A 1 iquid helium cooling system combined with an 0xford 3120 temperature controller provided a wide specimen temperature range from $4^{\circ} \mathrm{K}$ to $320^{\circ} \mathrm{K}$. 
Specimens with dimension $4.5=15$ w were cut from boron ion implented silicon wafers, and then were chemically etched with $\left(\mathrm{HMO}_{3}+\mathrm{HF}\right.$ ) solution to eliminate the dangling bonds on the cleaved surface. Then, the specimen was loaded on a teflon holder which was inserted into a quartz tube located at the center of the pole pieces of the magnet.

Many tr icky operational skills such as obtaining good vacuum anu temperature stabilịty, etc., are necessary to record a good EPR spectrum. A good reference for EPR experimental techniques is Poole. 116 


\section{EXPERIKEMTAL RESULTS}

\subsection{The Discovery of Small nerphous Zones}

The most interesting and crucial question in the study of the anorphization by light ion damage, is to determine whether or not the formation of amorphous silicon is related directly to individual ion tracks. The direct formation of amorphous zones by individual heavy ions has been proved. 25 small anorphous zones produced by the cascade have even been observed for intermediate ion mass such as silicon self implantation. ${ }^{58}$ It is still unclear whether lighter ions such as boron produce amorphous zones after preaccumulation of a high enough point defect concentration or whether amorphous material forms spontaneously by nucleation and the sweeping of an interface through crystalline material containing more than a critical concentration of point defects.

In room temperature, $80 \mathrm{keV}$ low dose, $5 \times 10^{15} / \mathrm{cm}^{2}$, boron ion implanted silicon, no observable defects were found by TEM microstructural investigation even by high resolution lattice images of a crosssection. A typical lattice image for $5 \times 10^{15} / \mathrm{cm}^{2}$ room temperature specimen is shown in Fig. 22. The lattice image is not in uniform and smooth contrast as would be the case for a perfect crystal, but is full of light and dark areas of 2 or 3 lattice distances in diameter. Because the thickness of the thin foil observed under TEM must be at least $200 \mathrm{~A}$, the point defect slusters such as divacancies, diinterstitials and 4-vacancies clusters etc. which are buried inside the foil are much smaller than the foil thickness. Although they probably 


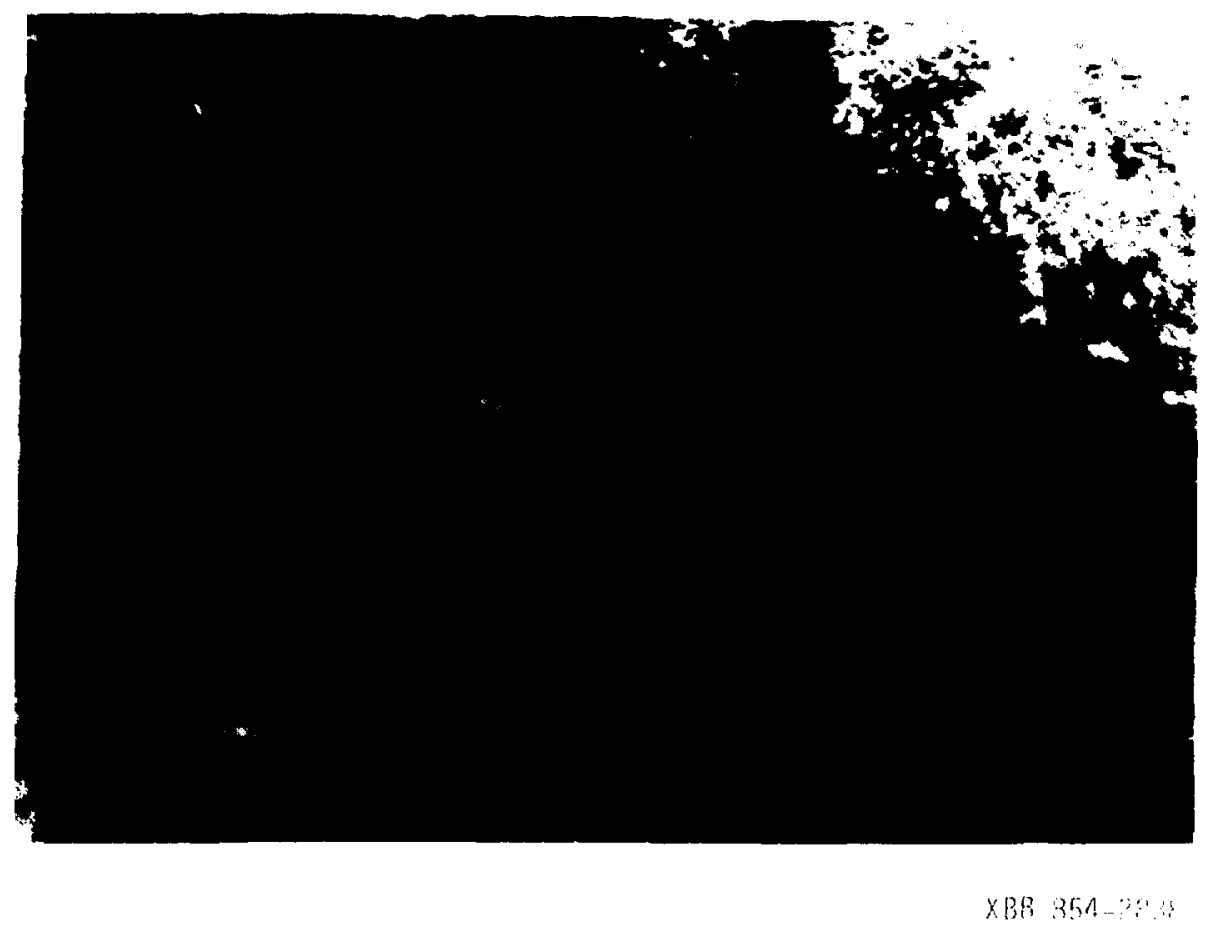


cause the uneven contrast they cannot be directly resolved. The strains of these point defect clusters would be expected to affect the localizes diffraction condition wid thus cause differences in local brightness of the lattice image.

A cross-section view of silicon implanted with $1 \times 10^{16} / \mathrm{cm}^{2}$ boron ions at room temperature and $80 \mathrm{KeV}$ shows that now small bright spots were observed near the depth of peak damage in Heak Beam Dark Field image (Fig. 23(a)). The corresponding Pright Field image is also shown in Fig. 23(b)). Although the WBDF image has much better resolution $(10-20 \AA)$ than the $B F$ image, the spots which are imaged due to diffraction contrast in WBDF, still cannot show the nature of the ion damage. High resolution lattice imaging (Fig. 24) of these spots revealed that they are damaged zones in which there is a loss of the periodic atomic arrangement in a region of about $20 \AA$ long and $10 \AA$ wide. The lattice image of these damaged areas could be interpreted as an amorphous zone or a very large point defect cluster in the matrix of silicon crystalline.

\subsection{The Identification of Amorphous Silicon by EPR}

A) though TEM xicrodiffraction or convergent beam techniques have been tried to identify the damaged zones created by ion implantation, they are not successfully methods due to the very small size of the damaged zones and the beam broadening effect. EPR which can detect amorphous silicon and specific point defects at the same time, seems to be the best candidate for this identification.

EPR spectra from a $5 \times 10^{15} / \mathrm{cm}^{2}$ room temperature boron implanted specimen with the magnetic field parallel to [110], [111] and [100] 


\section{BORON, 80KeV, $1 \times 10^{15} / \mathrm{cm}^{2}$, Rm Temp.}
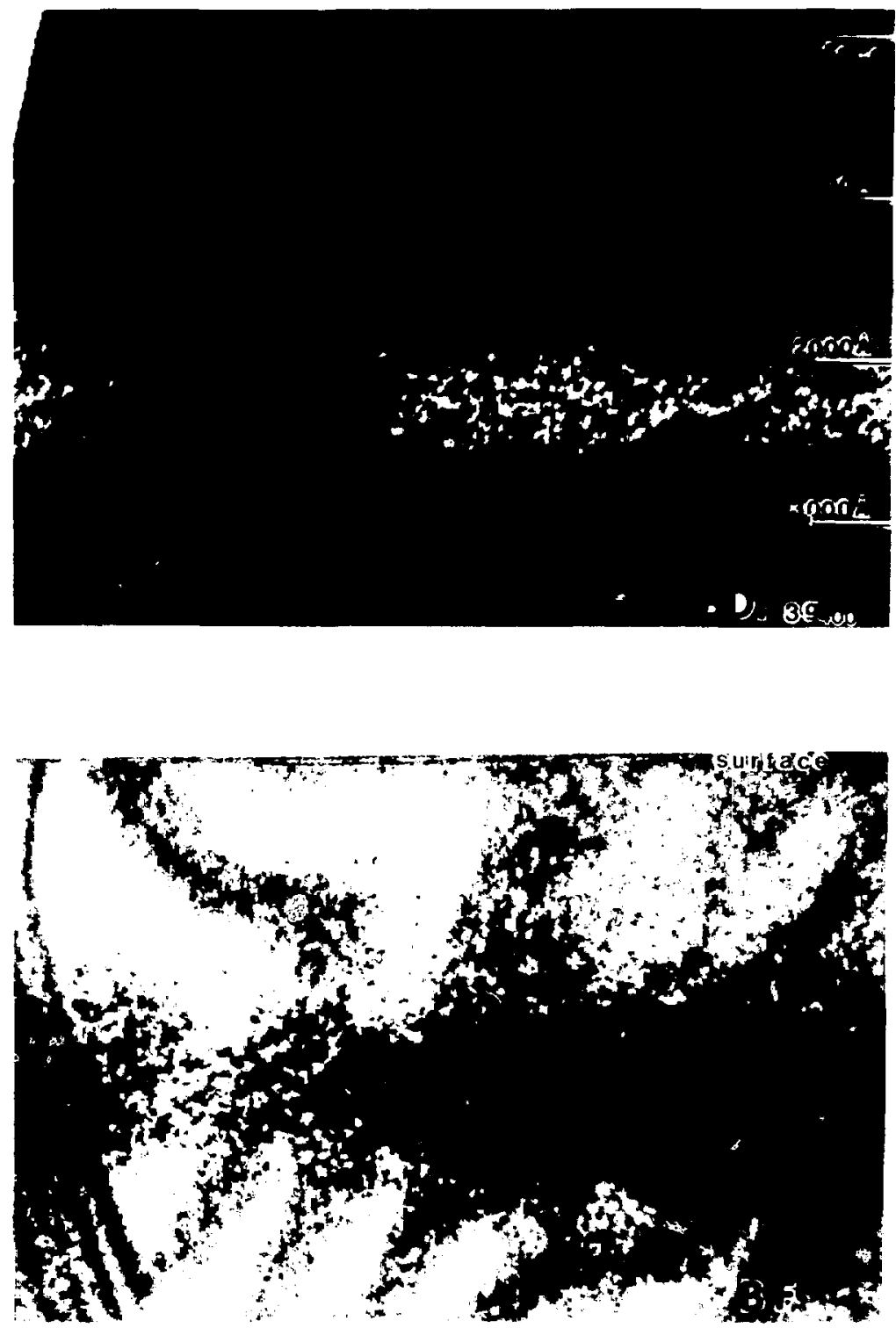

$\times 30.82-1449$ 


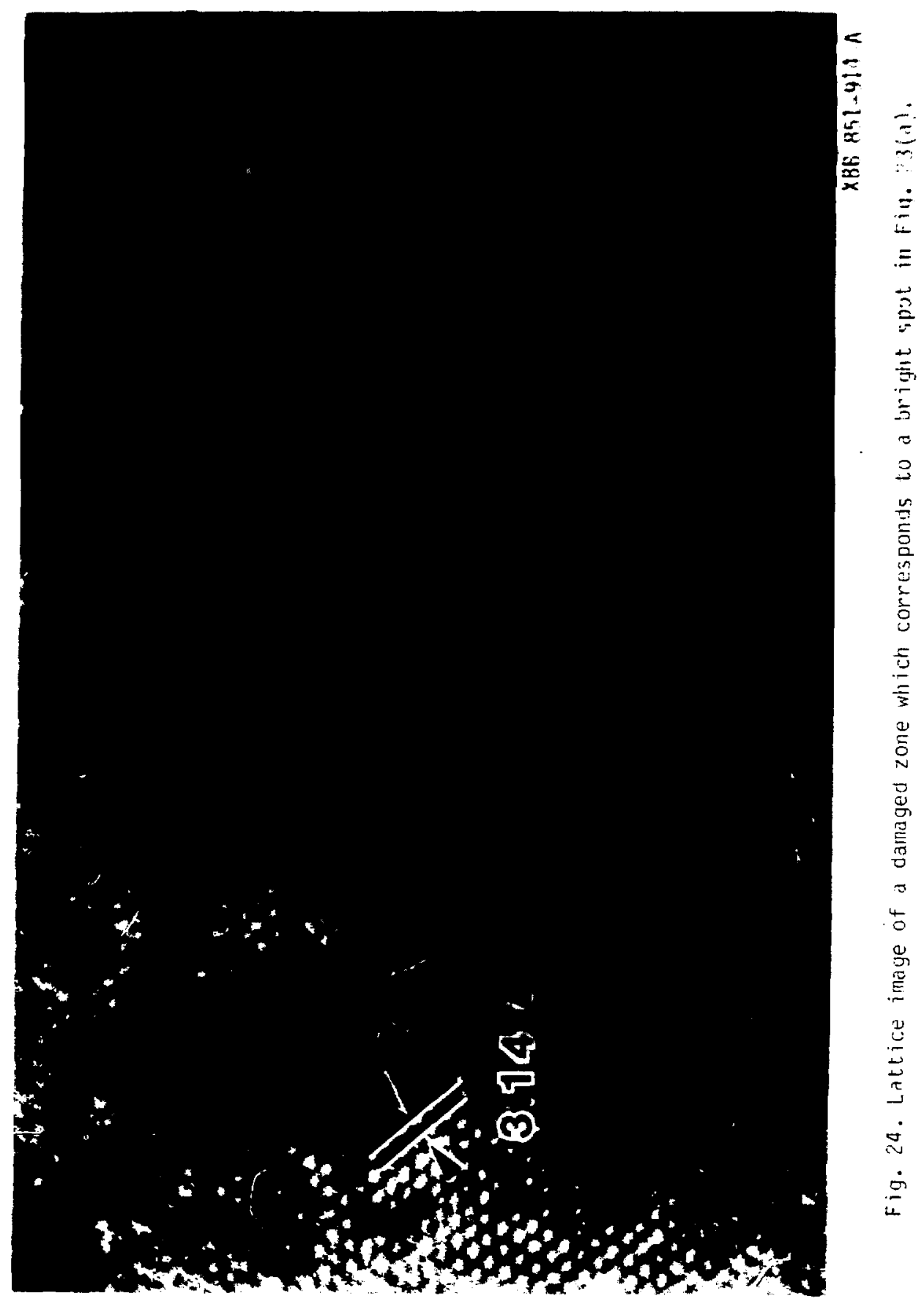


are shom in Fig. 25. The spectra were recorded at the optimu condition of $9.39 \mathrm{GHz}$ frequency, $10 \mathrm{~dB}$ attenuation, 2 Gauss modulation and $20^{\circ} \mathrm{K}$ temperature to obtain the strongest EPR signals. The spectra of Fig. 25 are orientation dependent showing that the signal was due to small unresolved anisotropic point defect clusters. No isotropic amorphous peak appeared in these spectra. However, an isotropic peak, which is the second peak from the left in the middle spectrum of Fig. 26, starts to appear in the $1 \times 10^{16} / \mathrm{cm}^{2}$ specimen. This isotropic peak becomes stronger for the $3 \times 10^{16} / \mathrm{cm}^{2}$ dose specimen which is shown as the lower spectrum in Fig. 26 . The isotropic peak in the spectra of $1 \times 10^{16}$ and $3 \times 10^{16} / \mathrm{cm}^{2}$ specimens is due to dangling bonds of random orientation and thus is associated with amorphous zones or regions. Therefore, the damage zone observed in the specimen of $1 \times 10^{16} / \mathrm{cm}^{2}$ dose (Fig. 24) was considered to be an amorphous zone buried in the crystalline material.

5.3 The Formation of Stacking Faults

With higher ion fluence $3 \times 10^{16} / \mathrm{cm}^{2}$, larger areas of amorphous silicon and two kinds of stacking faults were observed (Fig. 27). One kind of stacking fault was extrinsic bounded by $1 / 3<111>$ Frank partials, formed by the condensation of interstitial silicon atoms (Fig. 28). The other type of stacking fault which also lay on the (111) plane had a very small distortion (Fig. 29). The through-focus lattice Images of it are shown in Fig. 30 for defocus of Bol every step from : to $f$. The most interesting picture in this series is Fig. 30f, which is enlarges in Fig. 31. The white lat : re lines show the projection 


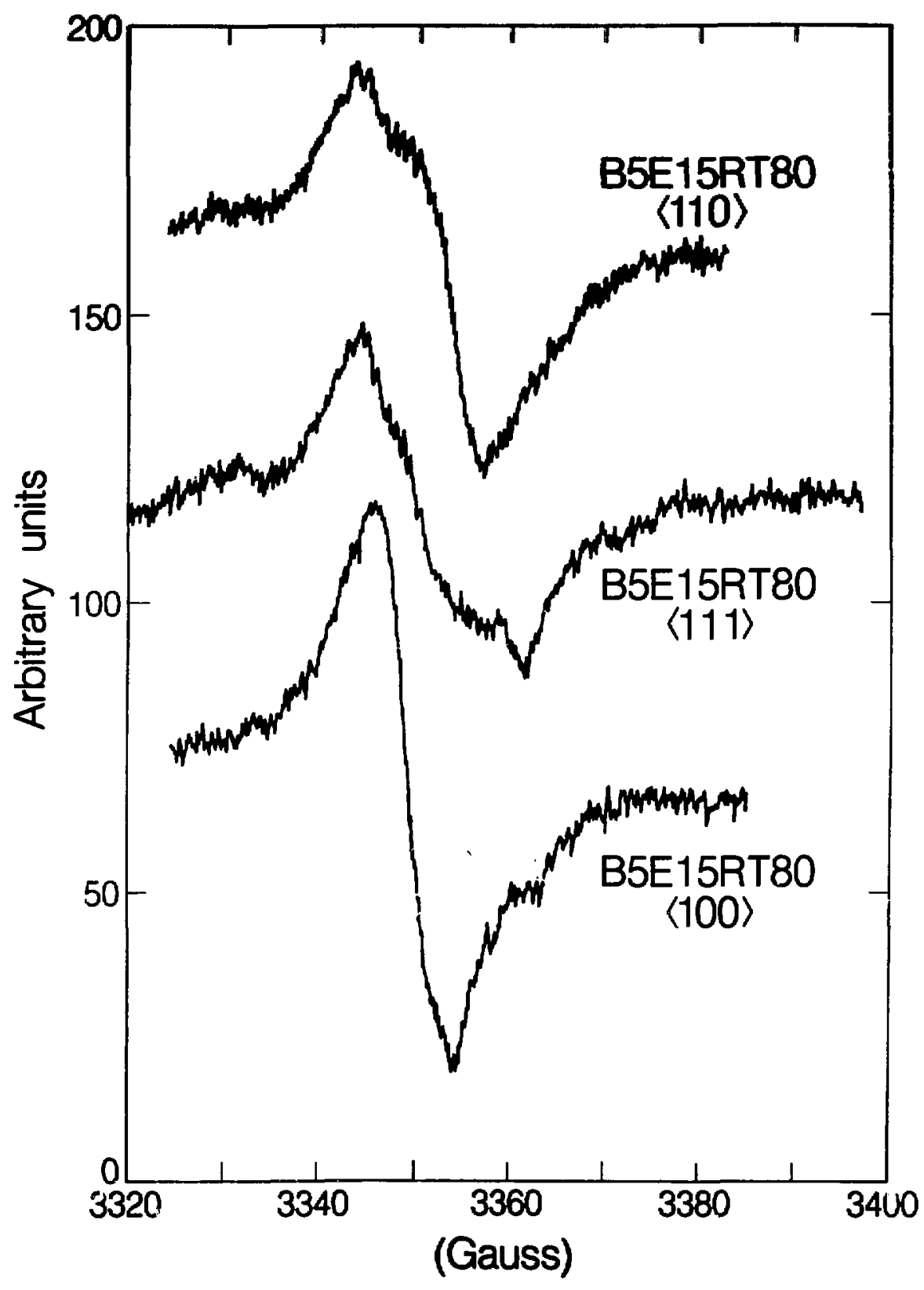

XBL 854.8845

Fig. 25. EPR spectra for the specimen of $5 \times 10^{15} / \mathrm{sm}^{2}$ boron jon implantation with $80 \mathrm{kel}$ at room temperature. The spectra were obtained with magnetic field parallel to <ll0s, <lils and $<100>$ respectively. 


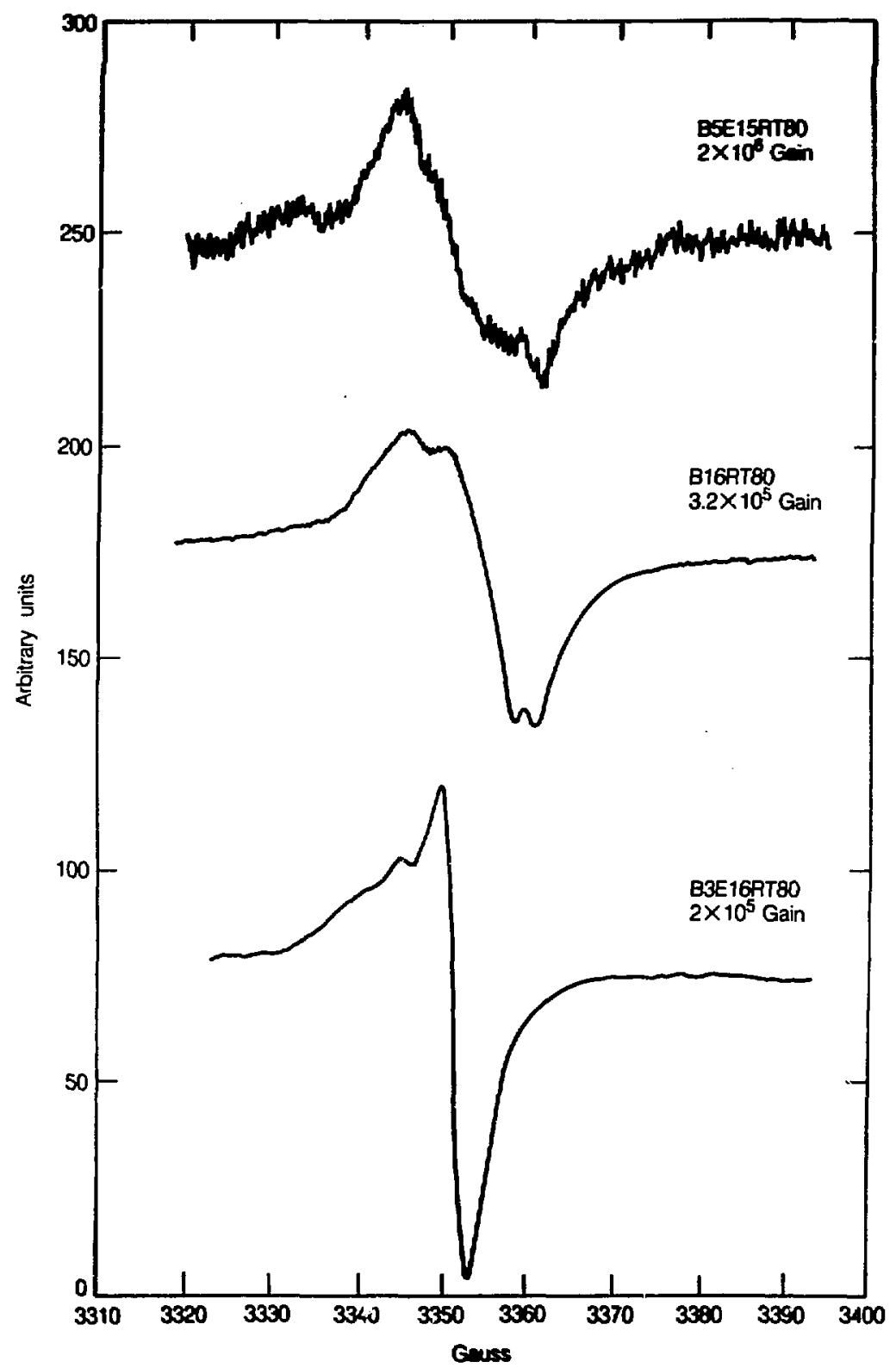

X논 -9519

Fig. 26. EPR spectra for the specians with $5 \times 10^{15}, 1 \times 10^{16}$, and $3 \times 10^{16} / \mathrm{com}^{2}$ boron ian (from top to bottom) $80 \mathrm{keV}$ implantation at room temperature. 


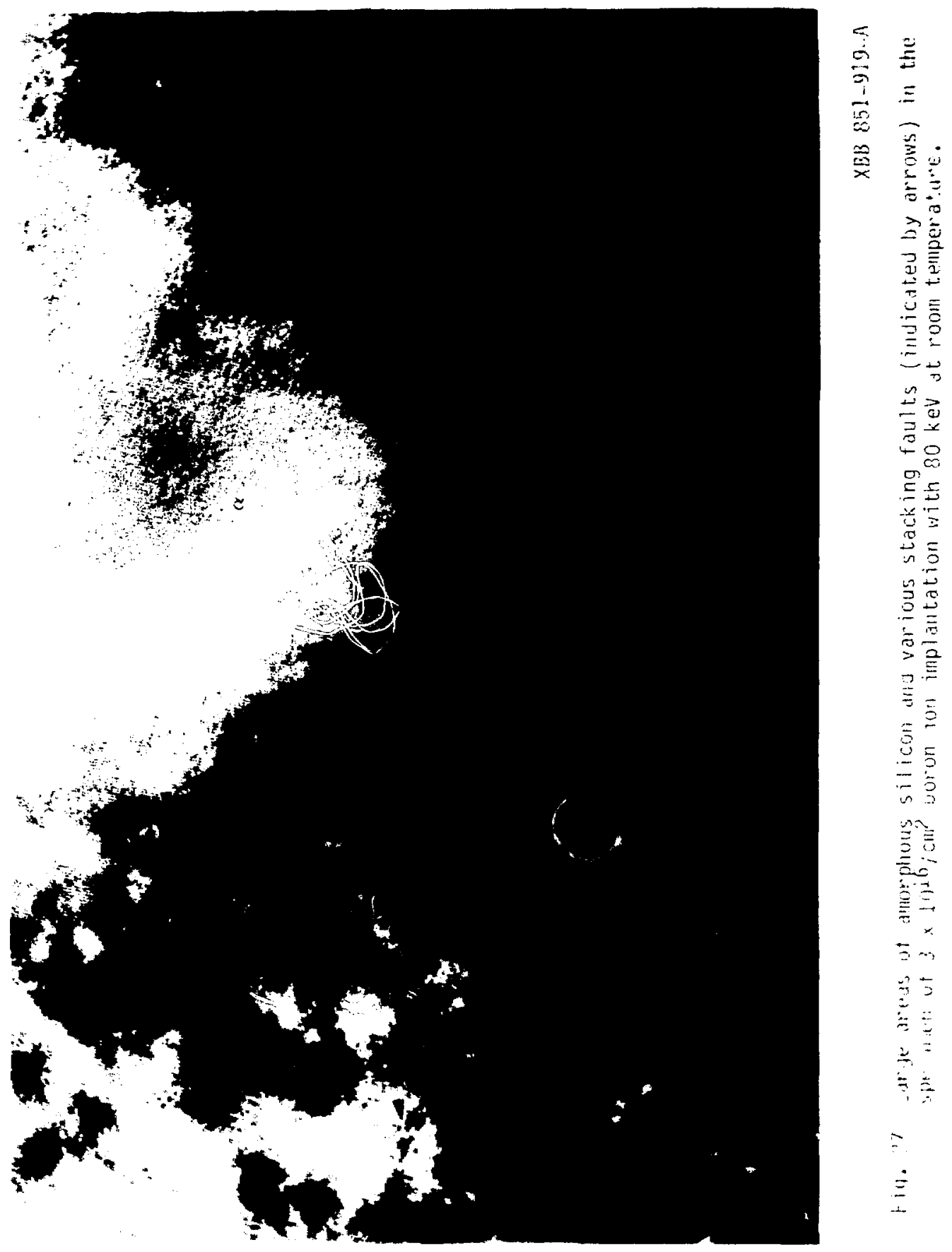




\section{- $\quad 88$}

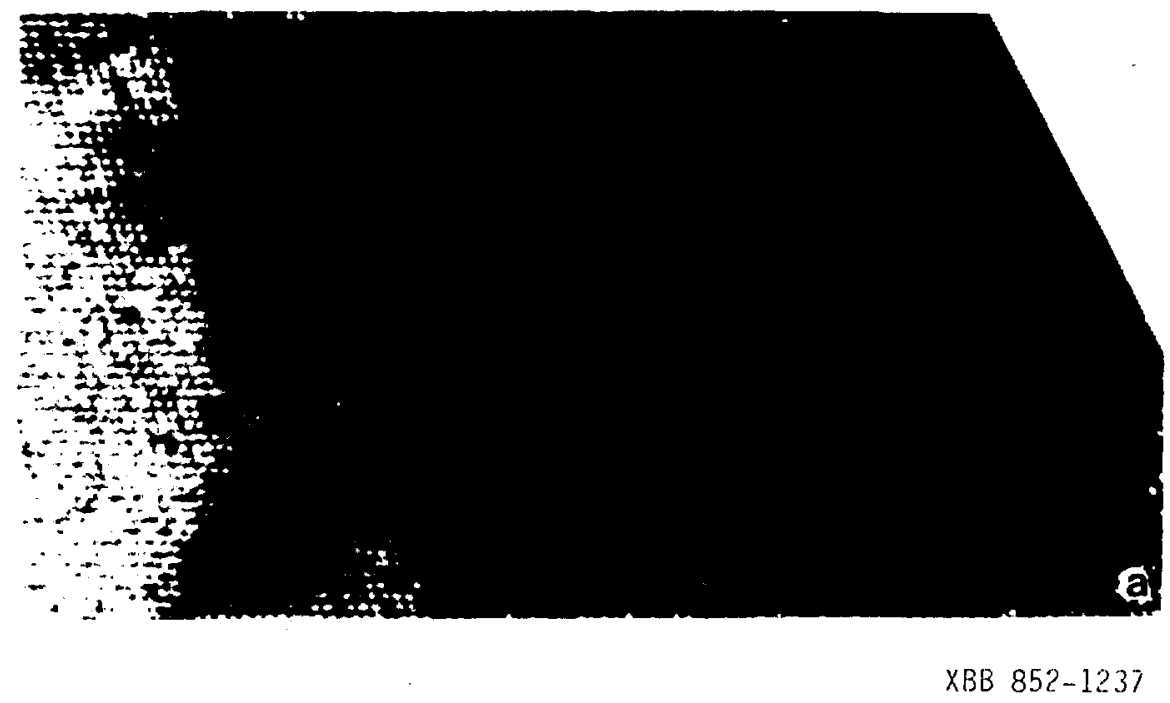

Fig. 28. Extrinsic stacking faul i bounded by $1 / 3<111>$ Frank partials in tive specilien shown in Fig. ?.7. 


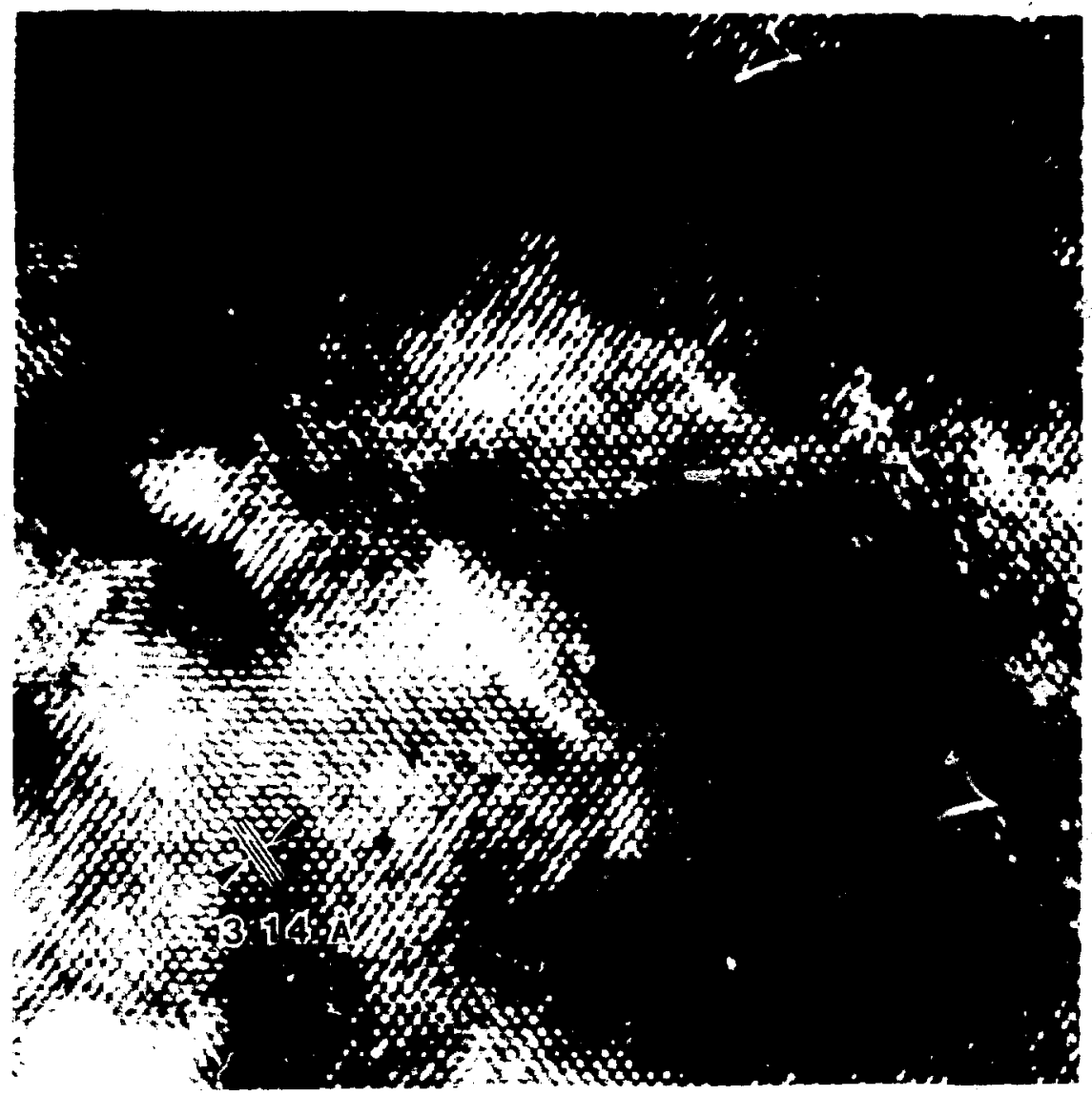

YPB $851-9 ? 4-1 ?$ 

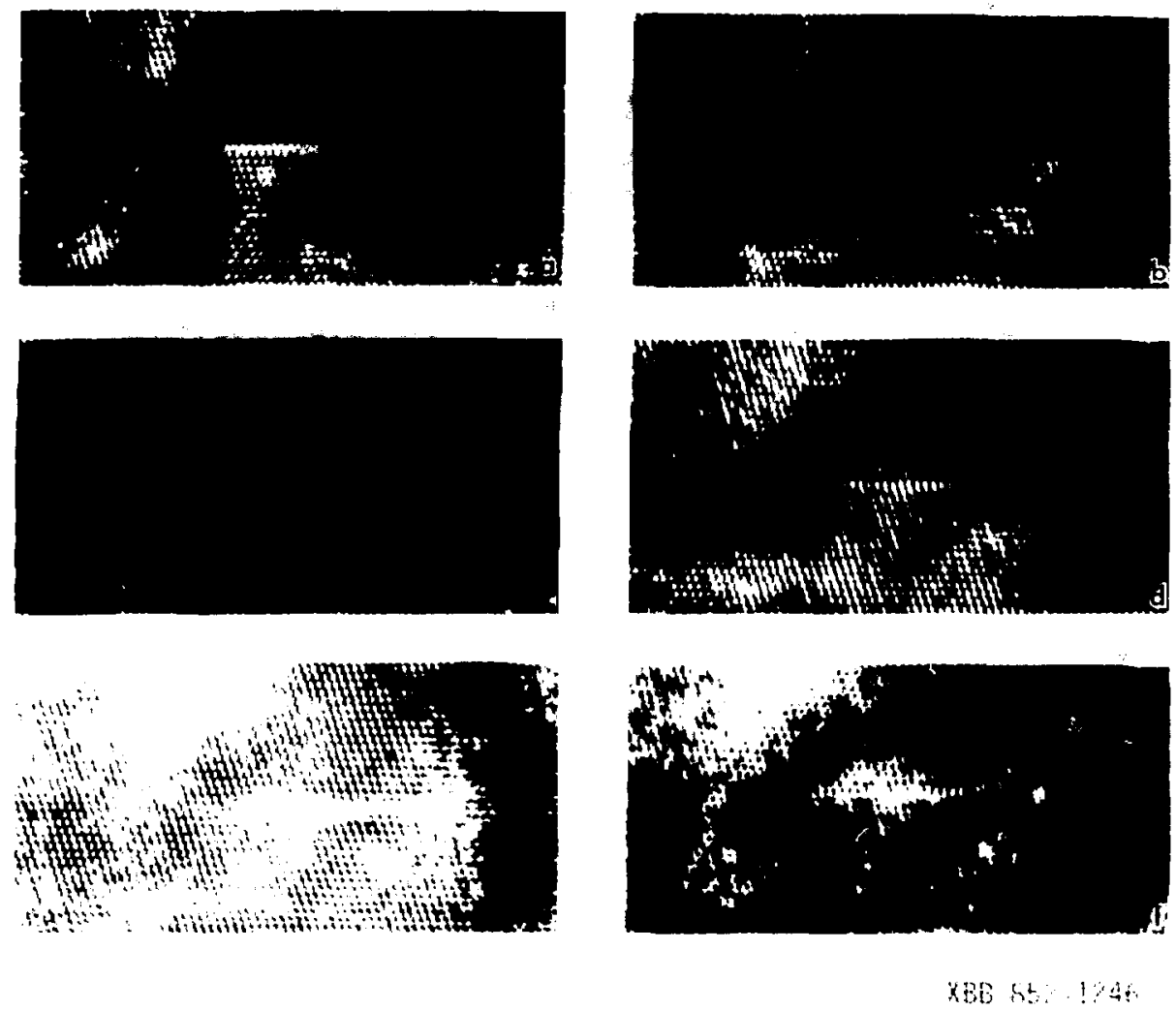

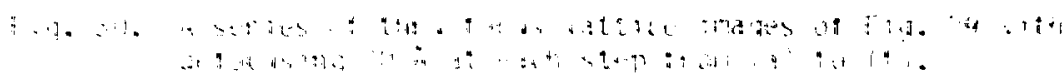




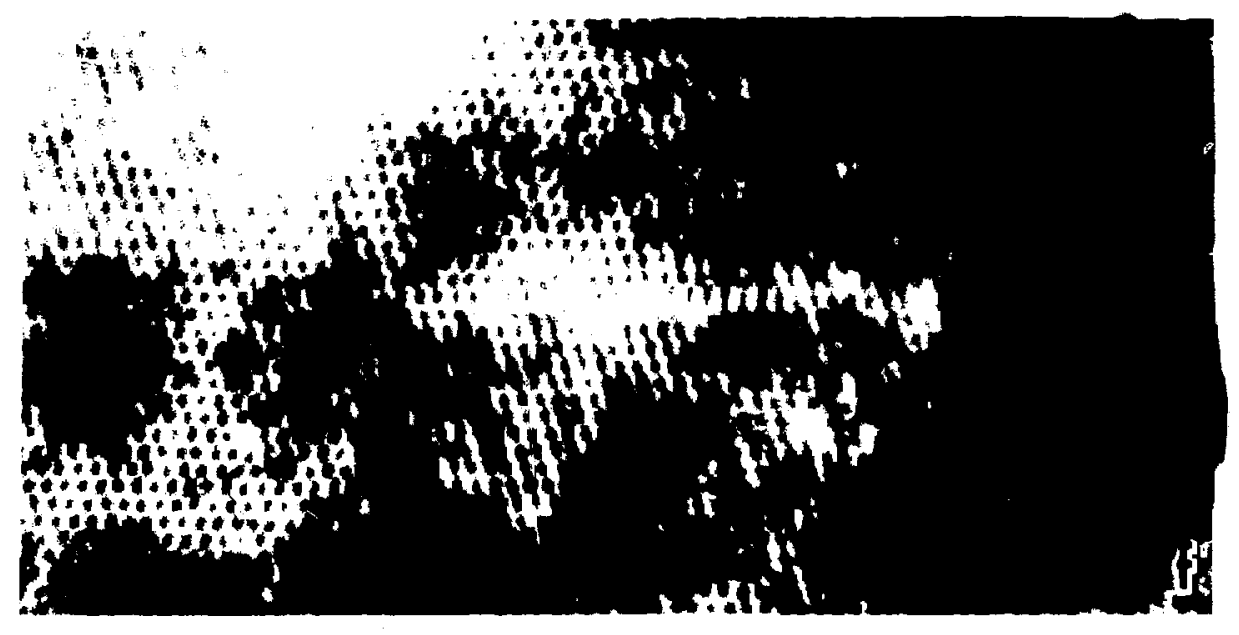

X68 $35 \%-1 \% 45$

Fig. 31. The eniarged latace hage nf Fia. zult'. 
of six fold "chair" type diamond cubic lattice in $\langle 110\rangle$ orientation. The aark spots represent the empty spaces between lattice atom rows (the opposite contrast as that in Fig. 30a). The identification of this defect would require structure modeling and computer simulation. However, considering that the concentration of boron $\left(\sim 1.9 \times 10^{21} / \mathrm{cm}^{3}\right)$ near the average projected range exceeds the maximum solubility of boron in silicon $\left(\sim 4 \times 10^{20} / \mathrm{cm}^{3}\right.$ at $\left.1000^{\circ} \mathrm{C}\right), 117$ it is probably boron or boron compound precipitation. With defocusing $80 \AA$ from Fig. 29, the arrows show areas that appear to have amorphous contrast in Fig. 32 , these same areas in Fig. 29 still show lattice fringes. It suggests that because of the small size of the amorphous zones buried in the crystalline matrix, the amorphous zones can show amorphous contrast only at certain defocus condition. So, the complex contrast in Fig. 27 could be caused by buried amorphous areas at various depths in the crystalline matrix.

In the specimen with $1 \times 10^{16} / \mathrm{cm}^{2}$ boron implanted at $50^{\circ} \mathrm{C}$ with a heating stage connected to specimen holder, no amorphous zones were observed, only faulted planar defects were found as shown in Fig. 33 . Figure 33a is a (113) type stacking fault. Figure 33b and $d$ are (111) stacking faults similar to Fig. 29. Figure 33e shows a damage zone associated with a stacking fault at its right upper direction. Figure $33 \mathrm{C}$ is very interesting, it shows a pair of (111) stacking faults, one interstitial type and one vacancy type close together wich do not anaihilate. Figure 33c is enlargeo in Fig. 34. The pairing of an interstitial and a vacency fault on rearby layers is not expected without codlescence. The interstitial type stacking feult any be a 


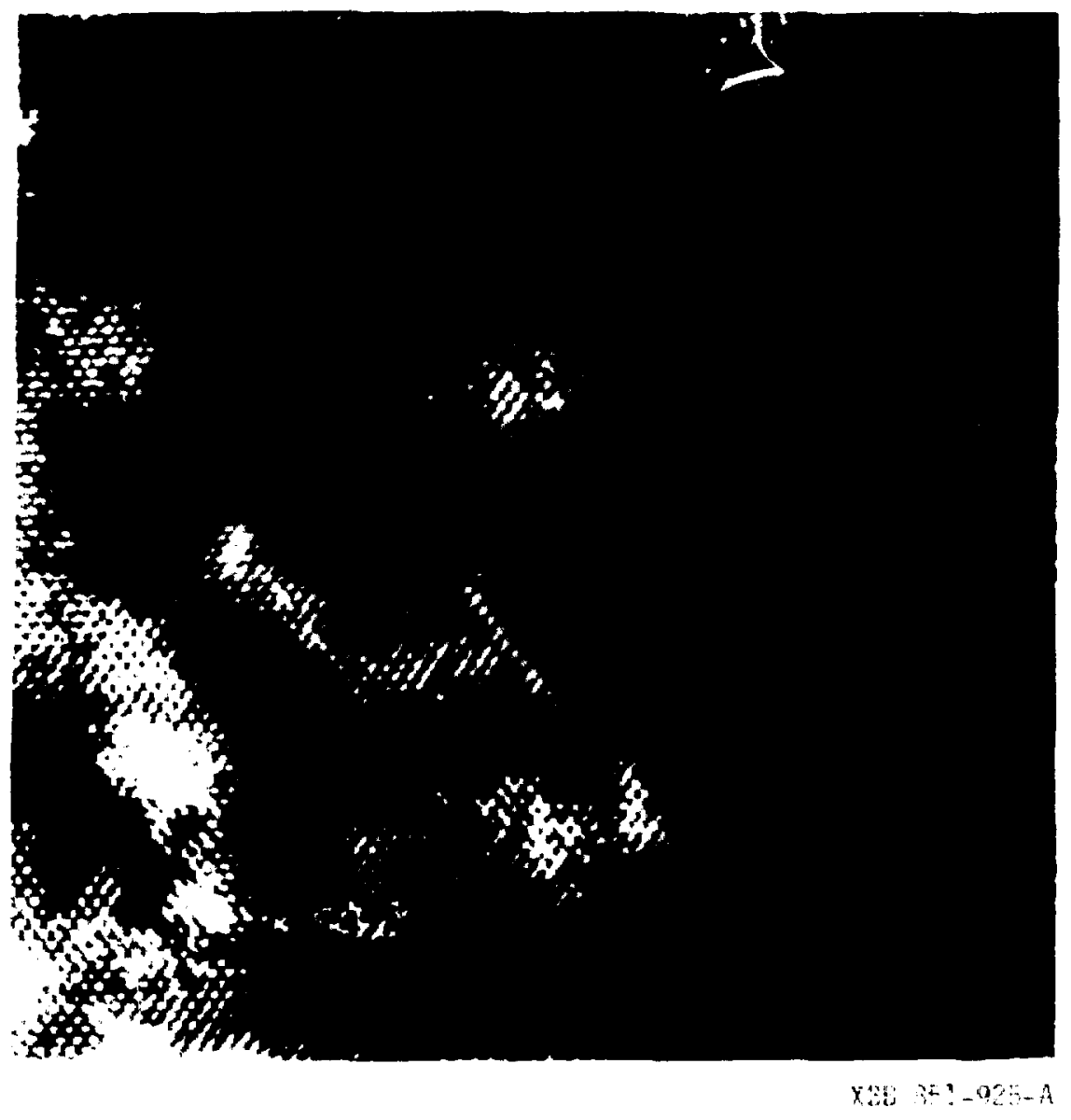

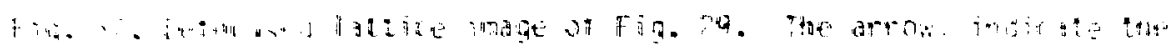

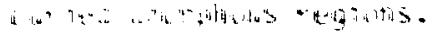




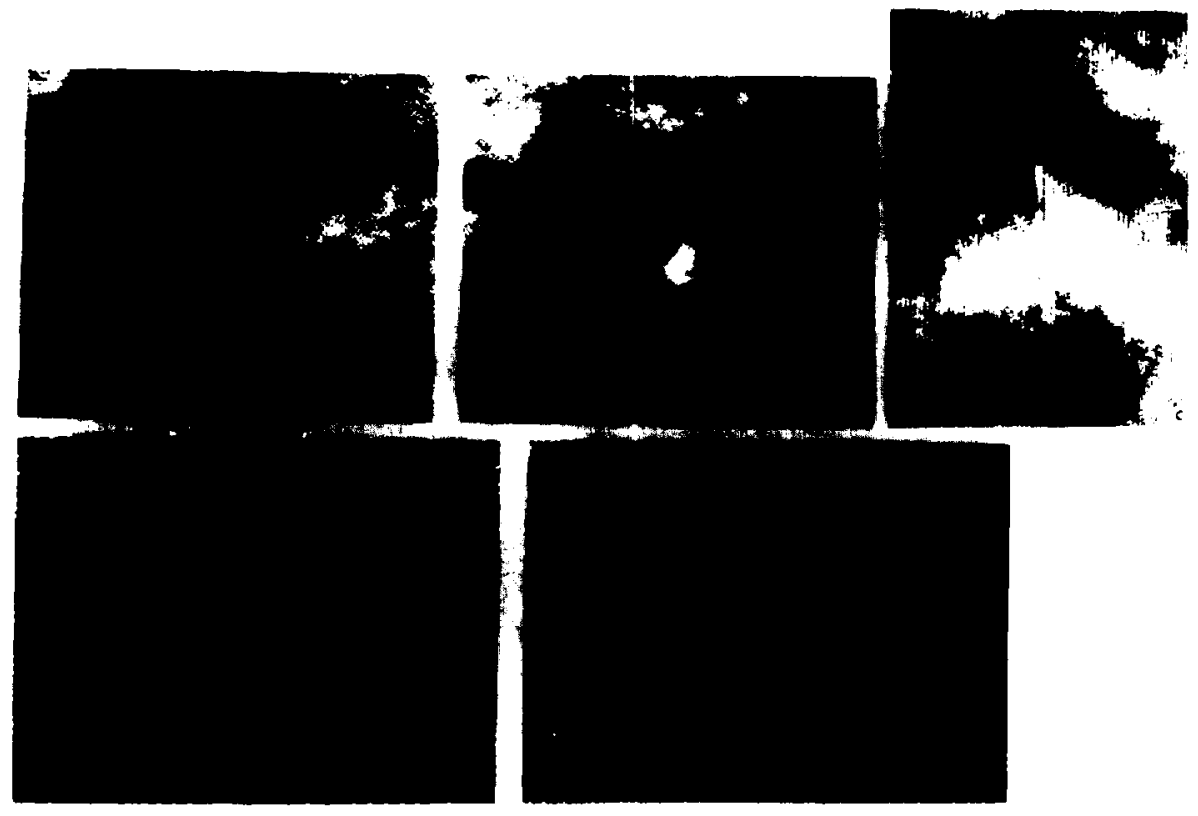

Bel: $964 \cdots 3$

$$
\text { a }
$$




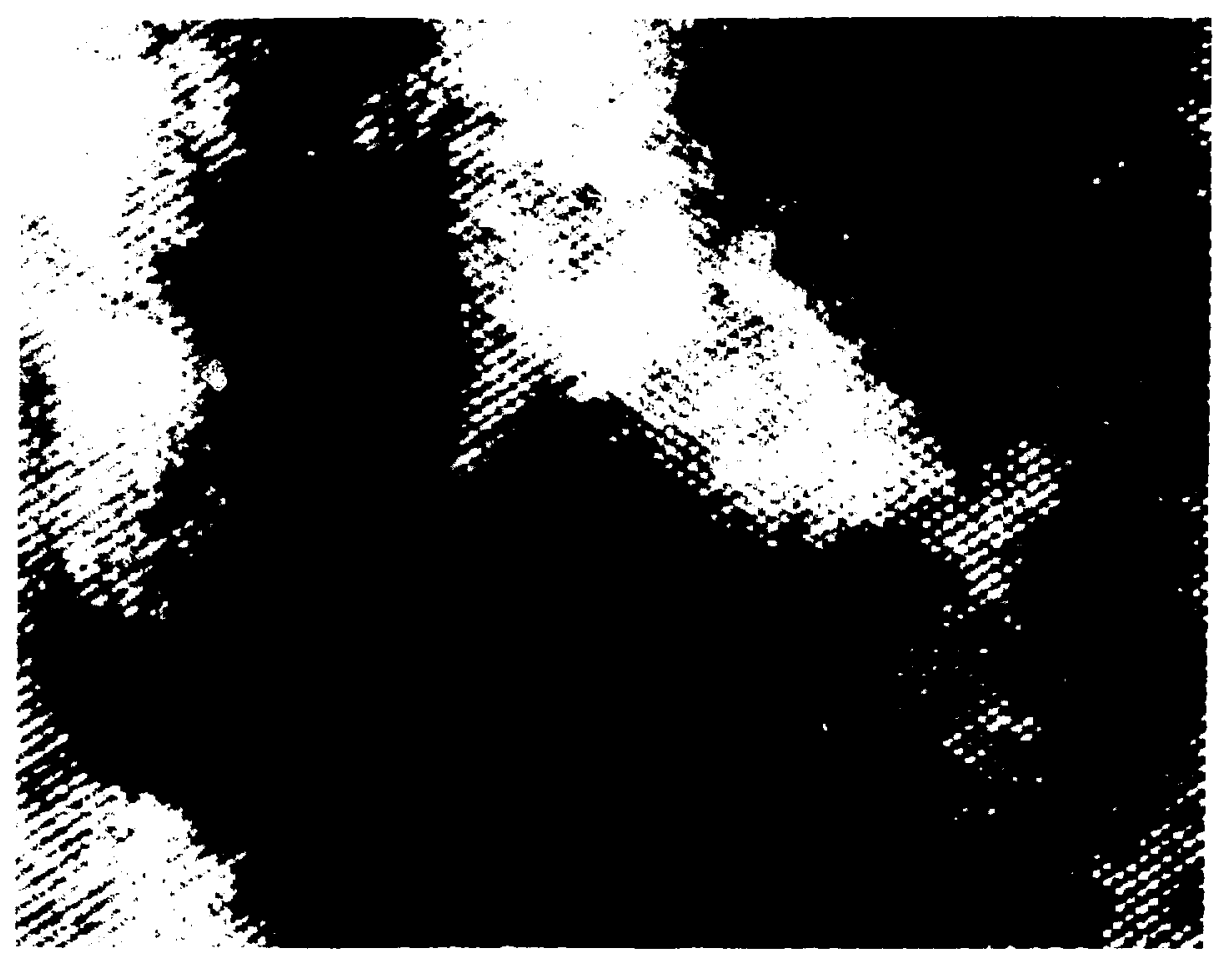

$390 \quad 304 \quad 3 \% 30$

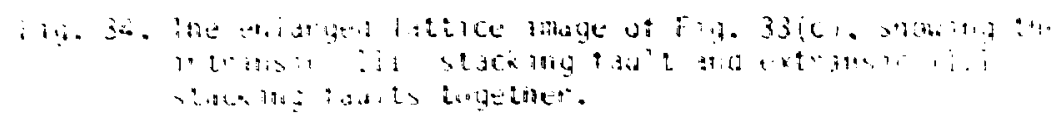


form of boron precipitate. Higher temperature implantation than room temperature was found to produce more stacking fault loops and fewer amorphous zones.

5.4 Effect of Predamage with Boron on the Formation of Amorphous Zones by Silicon

Figure 35 shows the result of implanting $5 \times 10^{15}$ boron ions $/ \mathrm{cm}^{2}$ at room temperature and $80 \mathrm{KeV}$ to produce a high concentration of point defects and small complexes without forming any amorphous zones followed by implanting a small fluence of silicon ions. The large $50 \AA$ diameter amorphous zones were formed at the end of the silicon ion tracks. On the contrary, if the predamage was done by silicon selfimplantation $\left(5 \times 10^{14} / \mathrm{cm}^{2}\right.$ at $\left.125 \mathrm{KeV}\right)$, only small amorphous zones $(-20 \AA)$ and (113) stacking faults (Fig. 36) formed by the following boron ion implantation $\left(5 \times 10^{15} / \mathrm{cm}^{2}\right.$ at $\left.80 \mathrm{KeV}\right)$. 5.5 The Formation of a Continuous Amorphous Layer

Even a very high dose $\left(3 \times 10^{16} / \mathrm{cm}^{2}\right)$ boron ion implantation cannot produce a continuous amorphous layer in silicon wafers at room temperature. The WBDF and BF images of this specimen are showed in Fig. 37. Oniy specimens either implanted at liquid nitrogen temperature or at a high beam current (-2 ma) can create a continuous amorphous layer. Figure 38 shows a buried amorphous layer for the specinen of $5 \times 10^{15} / \mathrm{cm}^{2}$ boron implantation with $100 \mathrm{KeV}$ at liquid nitrogen temperature. The lattice inages clearly shomed the formation of small isolated amorphous zones within the transition region of the anphous-crystalline interface. Figure 39 shours a defocus series 

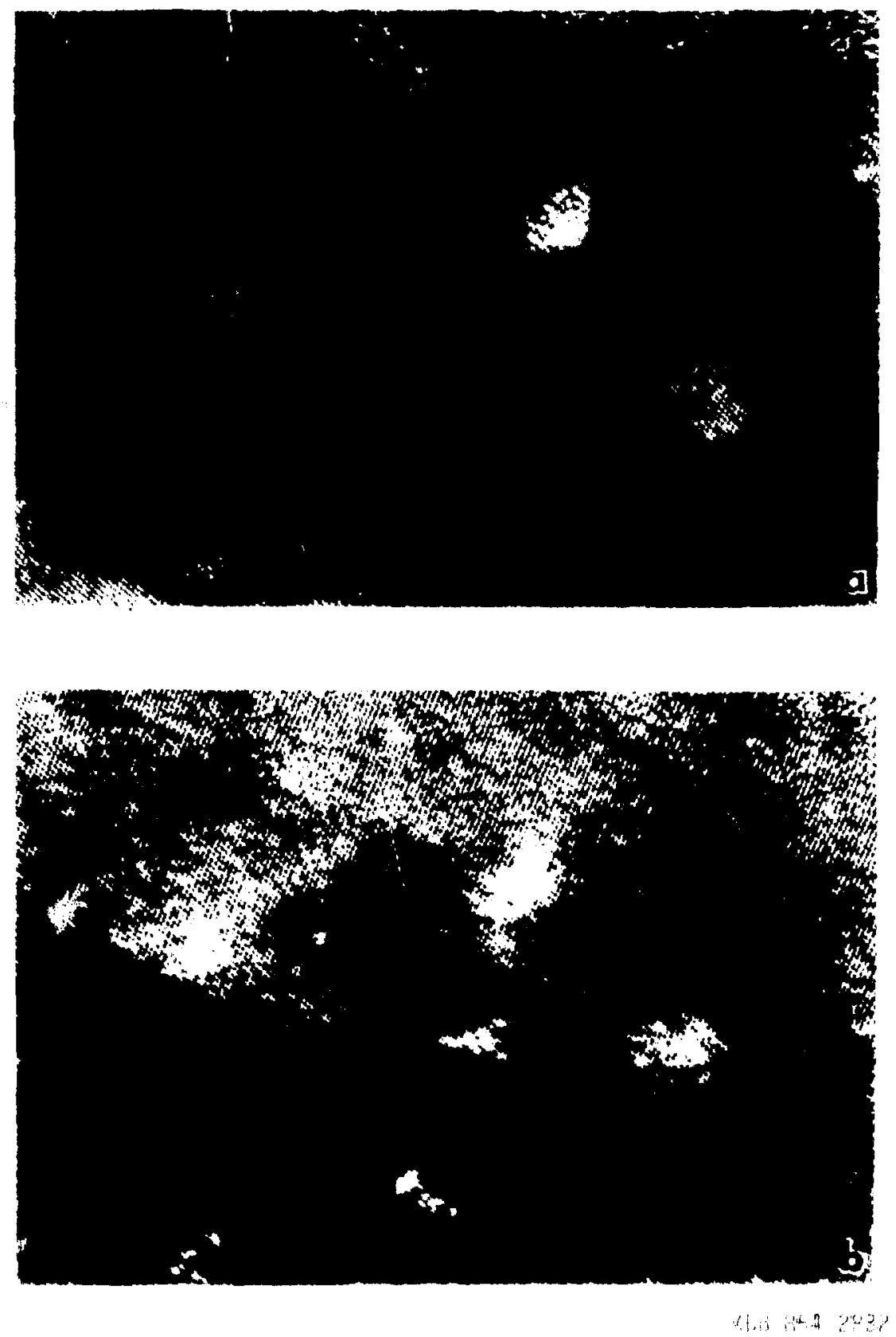

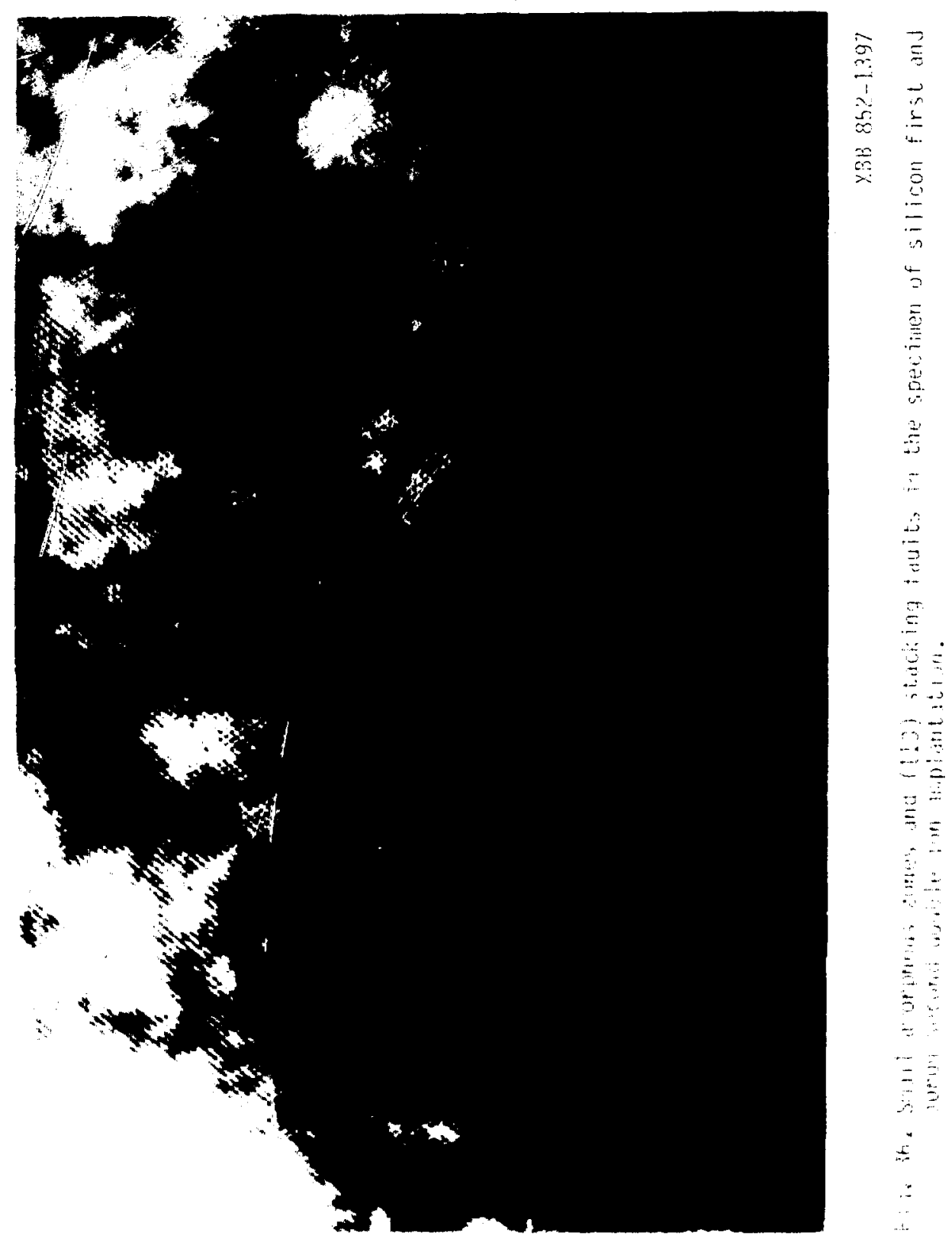


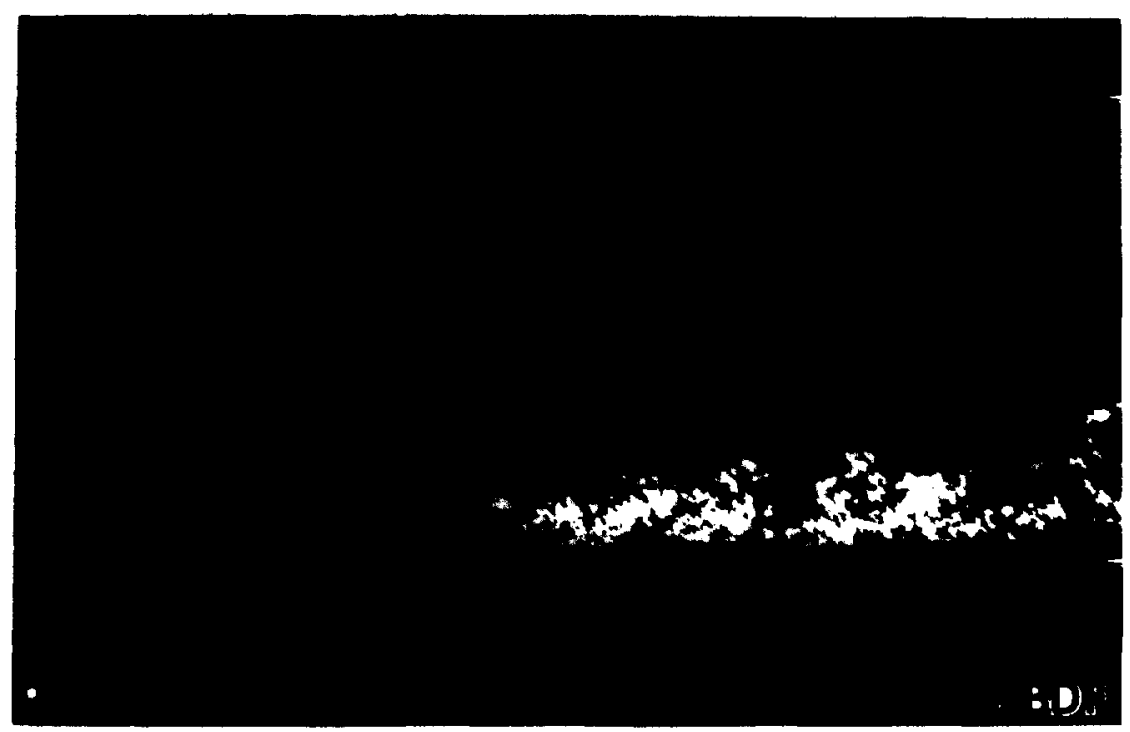

B $3 \times 10^{16} * 80 \mathrm{kev} * \mathbf{R m}$ temp

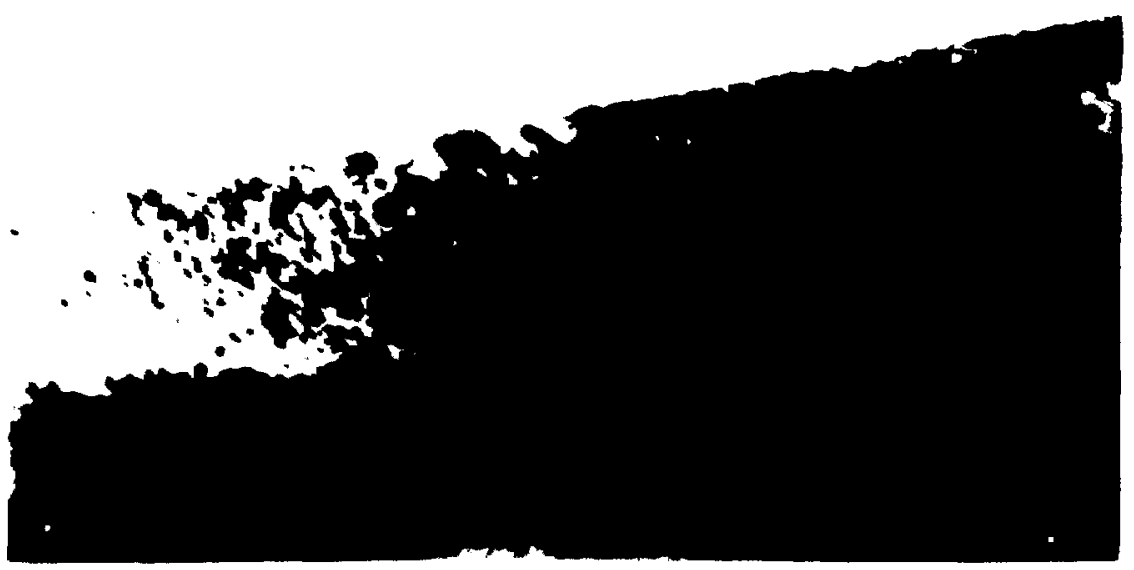




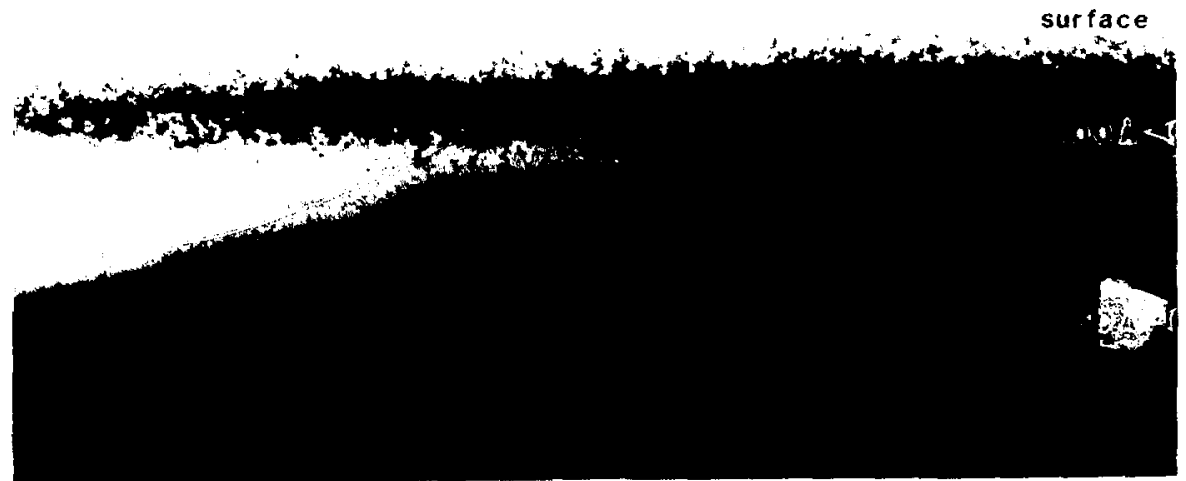

$\times 9 B 95499,97$

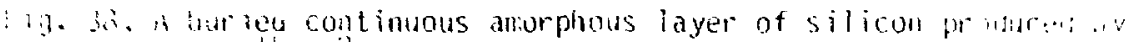

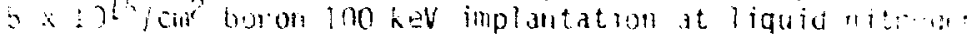
botipe ature. 

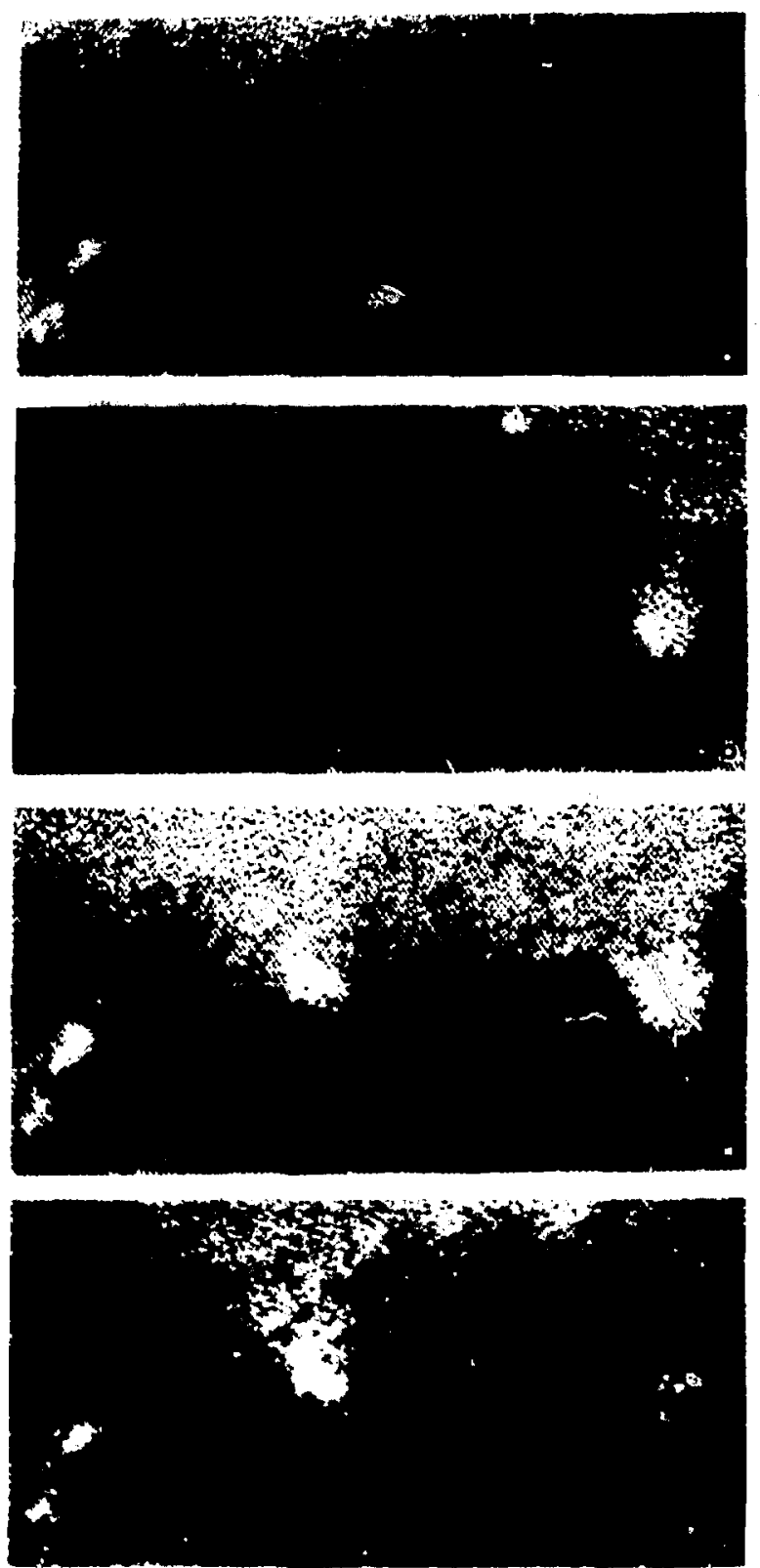

M०18 B54-2899

1 to

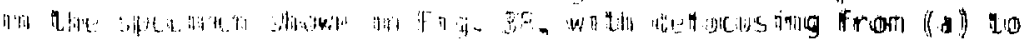
(日). 
from 39a to 39d. A $1 \times 10^{16} / \mathrm{cm}^{2}$ boron implantation with $100 \mathrm{KeV}$ at liquid nitrogen temperature prodiced a continuous amorphous layer extenaing from the surface to a depth of $3450 \mathrm{~A}$ (Fig. 40) with a very sharp crystalline-amorphous interface. The high beam current ( 2 mA) boron $5 \times 10^{15} / \mathrm{cm}^{2}$. implantation $w$ ith $35 \mathrm{KeV}$ at a temperature around $35^{\circ} \mathrm{C}$ also produced a continuous amorphous layer from the surface to the depth of $1200 \AA$, however with a yery wide amorphous-crystalline transition region ( $750 \AA)$ (Fig. 41).

5.6 The Criticai Deposition Energy for Amorphization

A deenly buried amorphous layer starting at $4.9 \mu \mathrm{m}$ to $5.3 \mu \mathrm{m}$ depth away from surface was found in a specimen of $1 \times 10^{15} / \mathrm{cm}^{2}$ boron ion implanted silicon with $4 \mathrm{MeV}$ at liquid nitrogen temperature (Fig. 42c). The deposition energy density profile (also known as damage profile) calculated from Brice's theory is shown in Fig. 43. The critical energy for amorphization corresponds to the energy density at the crystalline-amorphous interfaces. It was estimated as

$3 \times 10^{20} \mathrm{KeV} / \mathrm{cm}^{3}$; ior boron $4 \mathrm{MeV}$ implantation. For boron $100 \mathrm{KeV}$ implantation (conbining Figs. 13 and 38) a similar calculation gives around $2.3 \times 10^{21} \mathrm{KeV} / \mathrm{cm}^{3}$ which is the product of $5 \times 10^{15} / \mathrm{cm}^{2}$ by $4.5 \mathrm{eV} / \AA_{3}$, which is the energy density at the $1100 \mathrm{~A}$ depth amorphouscrystalline interface with 100 KeV boron implantation at liquid nitrogen temperacure. The difference in the critical energy for low and high voltage implantation was almost one order of magnitude. The causes for this difference will be dis-ussed in the next chapter. 


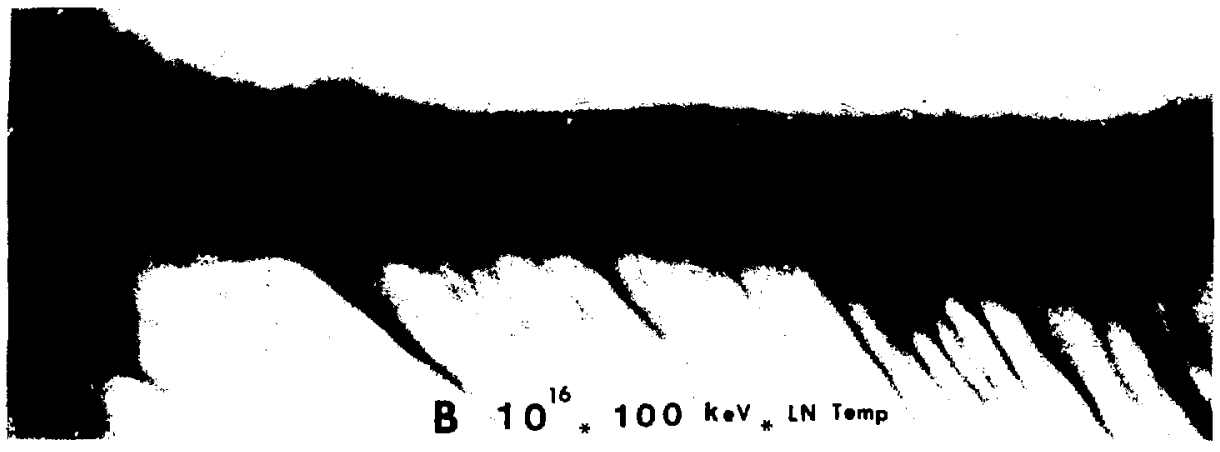

XBB 851-917

19. 40. A surfate continuous amorphous layer formed by $1 \times 10^{16} / \mathrm{cm}^{2}$ boron lick keV impiantation at liquid nitrogen temperature. 


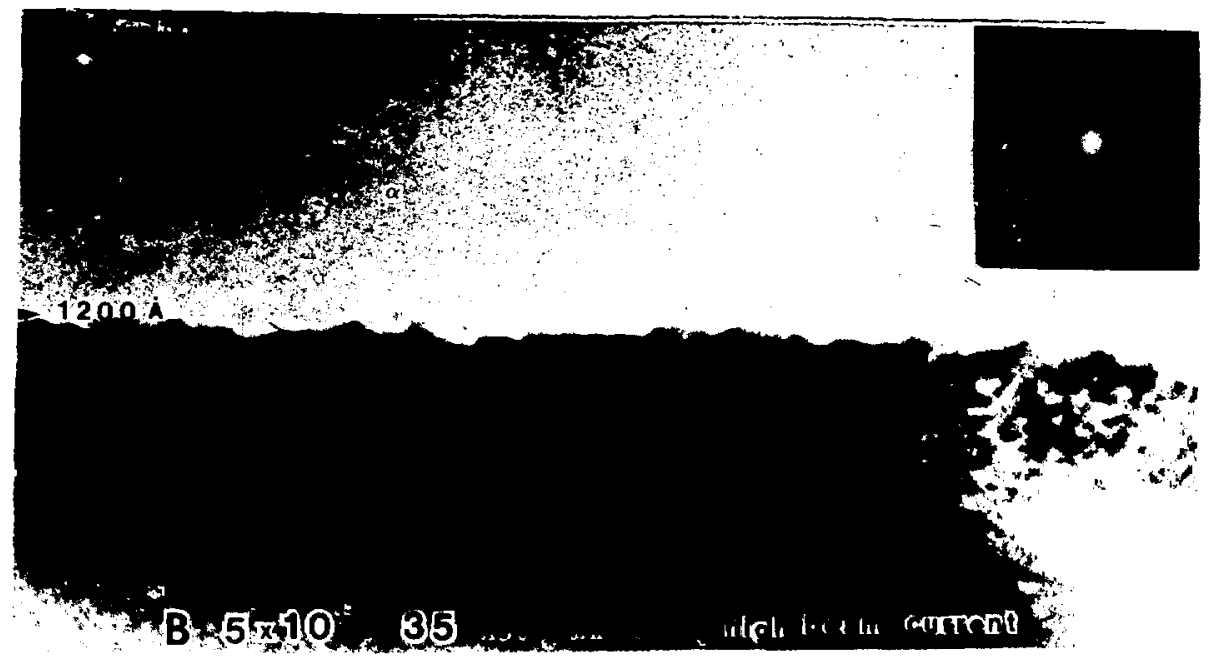

$X 98519$
9

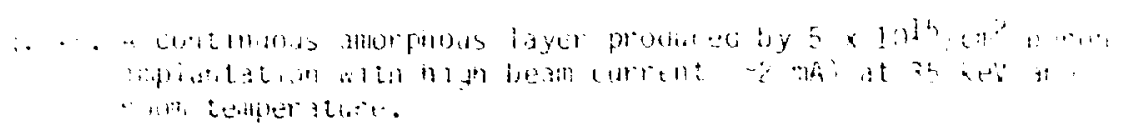


B 4 MEV $1 \times 10^{15}$
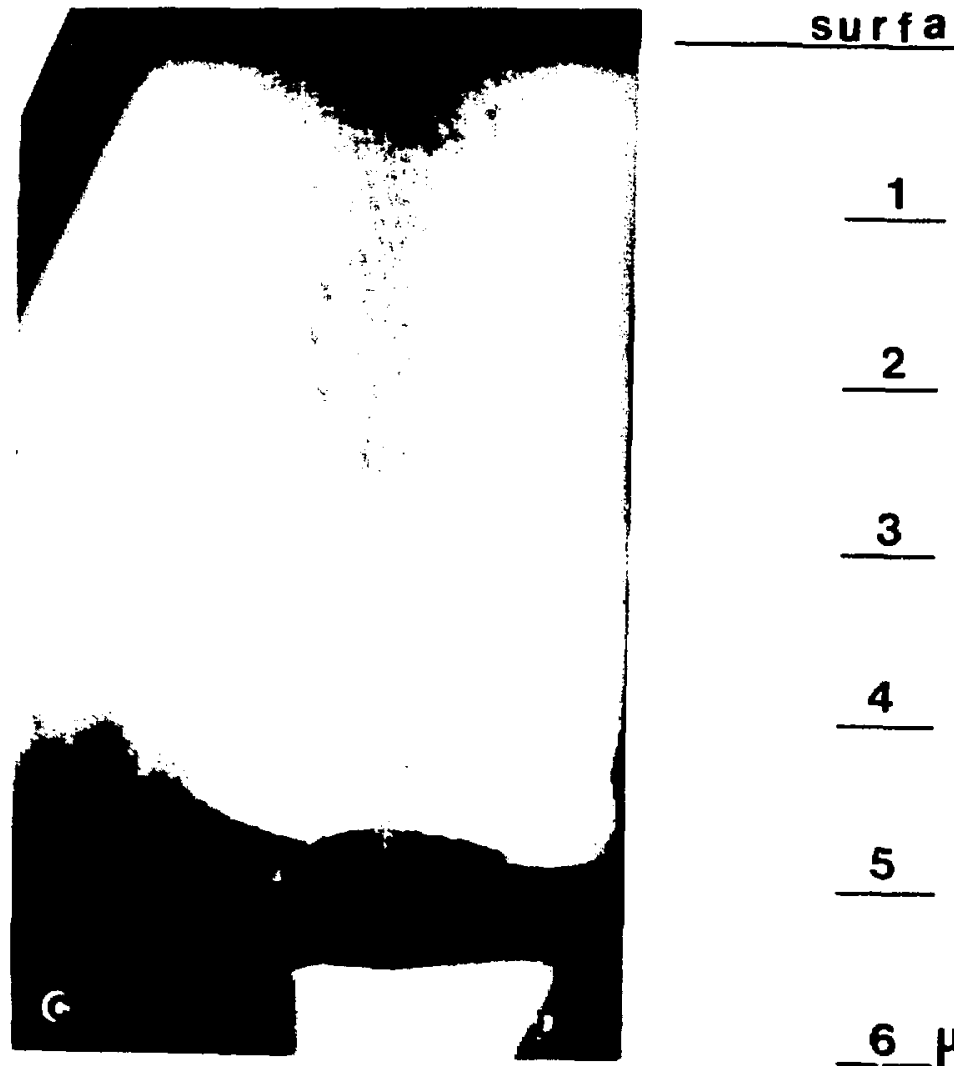

2

3

4

5

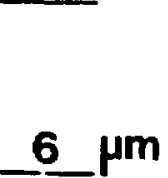

L. N cooling

no annea I 


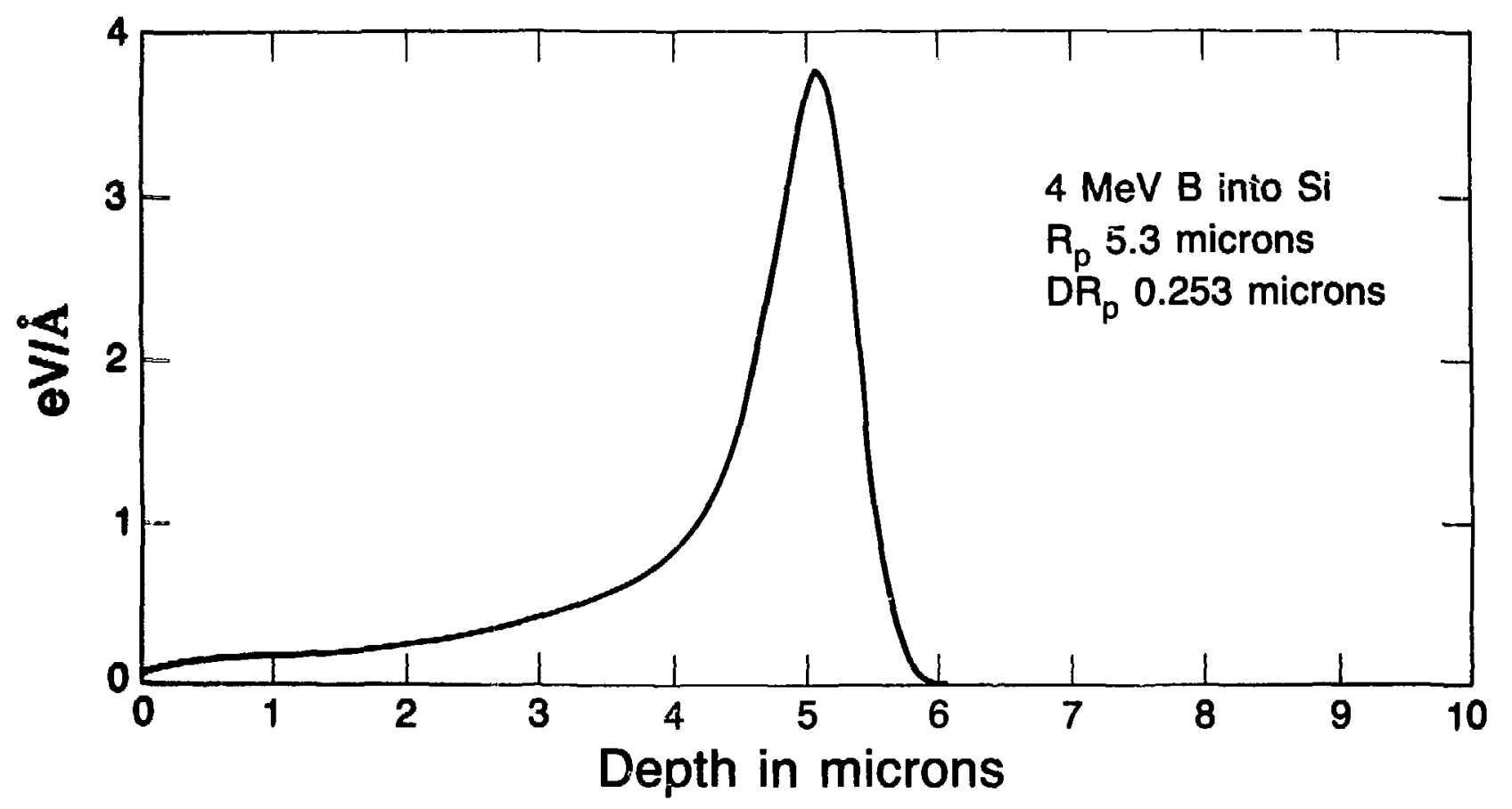

\&ั

Fig. 43. The damage energy deposition profile for $4 \mathrm{MeV}$ boron ion implanted silicon (calculated from D.K. Brice's formula). 


\section{DISCUSSION}

\subsection{The Formation of Amorphous Zone by Light Ion Damage}

The damaged zones of $-20 \AA$ in diameter (Fig. 23), which were found in the specimen of $1 \times 10^{16} / \mathrm{cm}^{2}$ boron ion implantation at room temperature, have been identified as amorphous zones by high resolution TEM lattice image and EPR spectrum. This result is consistent with the results of $I R$ reflection on boron ion implanted silicon (the first curve in the right in Fig. 4). A boron ion fluence of $1 \times 10^{16} / \mathrm{cm}^{2}$ is in the second stage of amorphization where amorphization is rapidly increasing. In all the specimens boron ion implanted at room temperature (Fig. 24), or at liquid nitrogen temperature (Fig. 39), and after double ion implantation (Figs. 35 and 36 ), small amorphous zones were observed in TEM lattice images. These results strongly suggest that the formation of amorphous zones occurs near individual ion tracks even for light ions. Thus amorphization during the second stage is due to the cascade. It is not due to the "collapse" of crystalline structure simply through point defect accunilution. The formation of amorphous silicon by light ion damage is therefore suggested to consist of two stages: first stage, the point defects or point defect clusters are accumulated to a critical concentration, at which an additional incoming light ion can create an amorphous zone individually. Then, the second stage begins. Larger volumes of amorphous material or a continuous amorphous layer are formed by the over lapping of these small amorphous zones. Therefore a heterogeneous nucleation model applies for light ions if only the second stage is considered. 
The overlapping, composite and multiple division models mentioned in Chapter 2 did not consider the diffusion and interaction of the point defects created during ion implantation, and should apply to amorphization at very low temperature at which all the point defects are immobile. These models are useful as a semi-quantitative way to estimate the critical dose for amorphous layer formation. They are not very helpful for an understanding of the mechanism of amorphization at higher temperature. The critical deposition energy criterion is another simplified semi-quantitative way of predicting the critical dose for amorphization by ion implantation without considering the diffusion and interition of point defects. It does not explain the effect of temperature of implantation or the effect of dose rate. 6.2 Amorphization Mechanism at Various Temperatures and Ion Masses

Owing to the effects of diffusion and clustering of point defects, the amorphization mechanism for light ion damage can be somewhat different at various temperatures. At liquid nitrogen temperature, the vacancies are basically immobile. Although some of the interstitials are known to migrate by athermal diffusion at $4^{\circ} \mathrm{K}$ (see Table 2, Chapter 3$)$, the other interstitials with charge (1-) or diinterstitials $\left(\mathrm{I}_{2}^{+}, \mathrm{i}_{2}^{0}\right)$ which have the activation energy for migration of $0.4,0.85$ and $1.5 \mathrm{eV}$, should also be nearly immobile at $77^{\circ} \mathrm{K}$. Therefore, little segregation of point defects or recombination through diffusion can be expected during ion implantation at $l$ iquid nitrogen temperature. With uniform scanning of an fion beam during implantation, the accumulation of points defects should be relatively uniform 
at liquid nitrogen temperature. In this case mechanism (A) described in Chapter 3 describes the amorphization process due to light ion implantation. For ion implantation close to absolute zero temperature (less than $4^{\circ} \mathrm{K}$ ) at which both vacancies and interstitials are immobile, the amorphization process can be described adequately by Gibbons' simple overlap model.

The diffusion and clustering of point defec:s during ion implantation at room temperature are expected to be much more pronounced than is the case at liquid nitrogen temperature $\left(D_{I}=3.16 \times 10^{-4} \mathrm{~cm}^{2} / \mathrm{s}\right.$, $D_{V}=4.15 \times 10^{-9} \mathrm{~cm}^{2}\left(\mathrm{~s}\right.$ at $\left.300^{\circ} \mathrm{K}\right)$. Figures $15(\mathrm{a})$ and $(\mathrm{b})$ demonstrate this, divacancies, 4-vacancy clusters, and dinterstitials are formed during ion implantation. Furthermore, extrinsic stacking fault loops with diameters of $50-150 \AA$ were found in the specimens of $3 \times 10^{16} / \mathrm{cm}^{3}$ boron implantation at room temperature. This shows relatively long range diffusion of silicon interstitials is occurring at room temperature and that there is enough mobility of vacancies to at least form small vacancy complexes containing two or more vacancies. This results in a higher concentration of small vacancy defects compared to interstitial defects particularly near the sirface where interstitials are not only lost to growing interstitial type dislocation loops but also to the surface. The existence of a high concentration of small vacancy aefects is also demonstrated by the formation of stacking fault tetrahedra near the surface in some cases after annealing. ${ }^{13}$ These results show that the distribution of point defects or small point defect clusters during boron ion implentation at room temperature 
is no longer as uniform as it is at liquid nitrogen temperature implantation. A nonuniform distribution of defects is also consistent with the evidence of Figs. 35 and 36 . The number of amorphous zones in Figs. 35 and 36 was estimated to be far below the number expected, if it is assumed that one amorphous zone is formed at the end of each ion track during the second stage of ion implantation. This suggests that the amorphization mechanism at room temperature is mechanism $B$ as described in Chapter 3.1. When point defects are mobile there is a less clear separation between stage 1 and stage 2 because of a nonuniform distribution of defect clusters throughout the volume. The critical density of point defect clusters necessary for formation of an amorphous zone at the end of an ion track may exist in only a fraction of the irradiated volume. If additional defect clusters are then formed preferentially in the strain field of already existing amorphous volume then most of the new amorphous materials may form adjacent to an already existing amorphous region, resulting growth rather than random coalescence.

Near the areas in which stacking faults are formed, no amorphous zories can be formed, because the stacking faults provide sinks for almost all the interstitial atoms necessary for the first stage accumulation in these regions. For $50^{\circ} \mathrm{C}$ inplantation, anorphous zones are rarely observed after boron ion implantatiun. More stacking fault loops (Fig. 33) and less anorphous material are observed in this case. Intrinsic stack ing fault loops (Fig. 3A) of $50 A$ in diameter were also observed in the specimen implanted with boron at $50^{\circ} \mathrm{C}$, due 
to higher mobility for vacancies at this temperature, and presumably loss of interstitial atoms in this region to some other nearby sink. Because of dynamic annealing at $\sim 200^{\circ} \mathrm{C}[16]$, at some temperature (between $25^{\circ} \mathrm{C}$ to $200^{\circ} \mathrm{C}$ ), no amorphous zones can be formed during boron ion implantation even with very high fluence, because of the high mobility of both vacancies and interstitials. The produced Frenkel pairs either recombine or segregate into large clusters such as dislocation loops. Therefore the critical concentration of diinterstitialdivacacy pairs for amorphization at the end of a boron ion track can never be reached even within a local area.

The amorphization mechanism for boron ion implantation gradually changes with implantation temperature; the evolution of the amorphization mechanism from very low temperature to the critical high temperature at which no amorphous region can be formed at all, is suggested to follow the sequence: (1; simple overlap model at temperature near $0^{\circ} \mathrm{K}$, (2) mechanism A at $77^{\circ} \mathrm{K}$, (3) mechanism B at room temperature $\left(\sim 300^{\circ} \mathrm{K}\right),(4)$ no amorphous material formed at all $\left(\sim 400^{\circ} \mathrm{K}\right)$.

The effect of ion mass on amorphization can be discussed qualitatively in a similar way as the effect of temperature. For boron implantation the accumulation of point defect clusters is thought to be more uniform than for a specimen implanted with a heavier ion such as silicon. Each silicon ion produces more localized danage than a boron ion. The formation of a few amorphous zones by the overlapping of two or three ion tracks in a short interval of time duing stage 1 is more likely for silicon because the required number of ower lapping 
tracks necessary to get an amorphous zone would be smaller. Once some amorphous zones are formed in this way, their strain fields would attract pcint defects, thus creating a non-uniform distribution for point defects (mechanism B). Ions of intermediate mass, al though not capable of forming amorphous zones initially at the end of a single ion track require much less accumulation of point defects or complexes before amorphous zones do start to appear. The diameters of the first amorphous zones formed in a specimen of silicon during self implantation was reported to be $30 \AA$ [58], when the first amorphous zones were formed in the specimen first implanted with boron to produce a high concentration of uniformly distributed point defects and then implanted with silicon; they were $50 \AA$ in aiameter (Fig. 35 ). This is consistent with the concept that amorphization is a two stage process and that a nigher concentration of point defects can be created without forming any amorphous zones when light ions are implanted. In this case the silicon ion tracks were terminating in a region where the point defect concentration was larger than that necessary for amorphous zone formation by silicon. Therefore each silicon ion formed a larger amorphous zone than it would have if only the critical concentration had been present.

If boron ion iniplantation below room temperature is assumed to be close to mechanism $A$, with ion mass increasing the intermediate ions (such as $\mathrm{Si}, \mathrm{p}$ ) produce amorphous silicon in a way corresponding to mechanism B. Heavy ions (Such as As) which can create amorphous zones inaividually without any preaccumulation of point defects, also produce 
an outer-shell region of point defects accumulation according to Morehead and Crowder's model (Fig. 10). As implantation temperature increases, the size of the amorphous zone shrinks due to point defect diffusion. At higher temperature, a small amorphous zone is surrounded by a relatively large area of point defect clusters during heavy ion implantation. Above a critical temperature, an amorphous zone created by a heavy ion would disappear spontaneously during implantation; only point defect clusters being left in the irradiated area. Therefore only stacking faults and various dislocation loops are expected to form at such temperature. For arsenic ion implantation, tangled dislocation loops were found at $200^{\circ} \mathrm{C}$ with no amorphous silicon formation [121].

6.3 The Out-Diffusion of Silicon Interstitials During Ion Implantation

The necessity of silicon interstitials for amorphization by ion implantation has been discussed in chapter 3.2.1.

The critical damage energy at the crystalline-amorphous interfaces in the specimen of $4 \mathrm{MeV}$ boron ion implantation at liquid nitrogen temperature were calculated as $3 \times 10^{20} \mathrm{KeV} / \mathrm{cm}^{3}$. This is almost one order of magnitude less than that of a typical specimen with $100 \mathrm{KeV}$ implantation (see Chapter 5.6). This can be explained by out-diffusion of silicon interstitials during ion implantation.

At 10 temperatures (such as liquid nitrogen temperature), at which vacancies hardly wove the build up of interstitial concentration under steady irradiation of the point defect pair production rate $P$ is approximated by: 118 


$$
\begin{aligned}
\frac{d C_{I}}{d t} & =P\left(1-C_{v}\right)\left(1-Z_{I V} C_{V}\right)-Z_{I V} M_{I} C_{V} I_{V}-Z_{I I} M_{I} C_{I}^{2} \\
& -Z_{I L} M_{I}\left(C_{L} C_{I L}\right)^{1 / 2} C_{I}-M_{I} C_{S} C_{I}
\end{aligned}
$$

where $M_{I}$ is the interstitial mobility and $Z^{\prime}$ 's are the site numbers of spontaneous reactions of each process. The first term in the equation is the effective production rate of free interstitials. The fraction of atomic sites at which primary damage can take place is $\left(1-C_{V}\right)$ where $C_{V}$ is the concentration of vacancies, and knocked-on atoms remain as interstitials only when they do not jump into regions of spontaneous recombination $Z_{I V}$ around a vacancy. The second term shows the recombination of interstitial atoms and vacancies. The third term is the formation rate of dinterstitials, and it gives the formation rate of interstitial clusters when dinterstitials are adopted as the nuciei. The fourth is the absorption rate of interstitials to interstitial loops (or stacking faults), where $C_{I L}$ is the concentration of interstitials which have already been absorbed to the loops of concentration $C_{L}$. The last term is the escape of interstitials to permanent sinks $C_{S}$ in concentration. A typical practical case of permanent sinks is the surface of a thin specimen. The value $c_{S}$ can be approximated from random walk theory as $c_{S}=(a / h)^{1 / 2}$ where a and $h$ are lattice constant and thin specimen thickness respectively.

For regular ion implantation, the ions penetrate inio target at a shallow depth from only one surface (a few hundred $A$ to a few thousand A). So, ion implantation usually creates a gaussian distribution of radiation damage with a peak near the silicon wafer surface. The 
out-diffusion of interstitials to the near surface during ion implantaticn is very similar to the out-diffusion of interstitials to both surfaces of a thin foil during electron irradiation. The $h$ in $c_{S}$ can be considered as two times the distance from the damage peak to the near surface for the ion implantation case.

The corresponding variation of vacancies is given hy

$$
\frac{d C_{V}}{d t}=P\left(1-C_{V}\right)\left(1-Z_{I V} C_{V}\right)-Z_{I V} M_{I} C_{V} C_{I}
$$

Exact analytical solution of the combination of Eqs. (6.1) and (6.2) cannot be obtained. Computer simulations can be made to find characteristic features of the variation of point defects. However, only semi-quantitative comparisori will be made here, because there are several athermal migration mechanisms for silicon self interstitials (such as Bourgoin machanism, ${ }^{119}$ or energy-release mechanism, ${ }^{96}$ ) and diffusion data for various charge state interstitials are still unclear.

The damage peak of $100 \mathrm{KeV}$ boron impl:ntation is about $2300 \mathrm{~A}$ away from surface (Fig. 13), while the damage peak of $4 \mathrm{MeV}$ boron iwn inplantation is around $5.1 \mu \mathrm{m}$. The proportion of $\mathrm{C}_{S}$ with $100 \mathrm{KeV}$ implantation to that of $4 \mathrm{MeV}$ implantation is about 4.7 , mich implies that the consumption of silicon interstitials by out-diffusicn to surface is 4.7 times faster for 100 KeV boron implantation than 1 MeV implantation. If comparison is made for the amorphous-crystal interface nearest to the surface instead of for the damage peak, the proportion of $C_{S}$ with $100 \mathrm{KeV}$ implantation to that of 4 MeV implantation 
is about 6.7. This is very close to the critical energy proportion for these two different voltages of implantation $7.3 \Leftrightarrow=2.2 \times 10^{21} \mathrm{keV} / \mathrm{cm}^{3}$ aiviaed by $\left.3 \times 10^{22} \mathrm{KeV} / \mathrm{cm}^{3}\right)$. Therefore, the increase oi critical deposition energy for amorphization at low voltage implantation is suggested to be due to the out-diffusion of interstitial atoms.

This phenomenon is also shown in the case of GaAs implanted with 450-KeV Se ${ }^{+}$at room temperature. ${ }^{120}$ The surface of GaAs still appears cryscalline in nature, while its deposition energy density is already nigher than the critical energy which was determined using the ceeper amorphous-crystalline interface. Many other exampler, are also found with a similar trend. ${ }^{121}$

\subsection{The Influence of High Beam Current Implantation on Amorphization}

If the production rate of Frenkel pairs created by ion damage is greatly increased with the other parameters being kept almost constant, the steady state concentration of interstitials and vacancies will be increased. This can be seen from Eqs. (6.1) and (6.2). Hence, the amorphization by ion damage is promoted by high beam current implantation. The increase of dose rate usually also raises the wafer temperature and thereby increases the mobility of interstitials and vacancies $M_{I}$ and $M_{V}$. This implies the increase of the values of the second, third, fourth, and fifth terms in Eq. (6.1) therefore causing a decrease in the concentration of interstitials. Anorphization with high beam current involves a competition between point defect production rate and the increase of mobility due to heating. The increase of wafer temperature by high beam current also enhances the segregation 
of point defect clusters as mentioned in Section 6.2. A wide transition region $(-750 A)$ with partial amorphization and many stacking fault loops or point defect clusters, was found for the specimen of boron implantation with 2 mA bean current (Fig. 41 ).

A continuous amorphous layer was produced by using high beam current ( $2 \mathrm{~mA})$ implantation and a good cooling system; however a wide transition region, which is the origin of profuse secondary defects, can be very detrimental to electrical properties of post-annealed specimens. More effective cooling would be necessary to solve this problem.

\subsection{The Development of Secondary Defects from Preliminary Defects}

As discussed in Section 6.2, once extrinsic stacking fault loops are formed, the surrounding region has not enough interstitials for amorphization and remains crystalline. The amorphous-crystalline transition region was observed to contain such small extrinsic stacking fault loops in the still-crystalline areas right after implantation. The stacking fault loops are mainly extrinsic stacking faults bounded by $1 / 3<111>$ Franck partials, which is also know extrinsic faulted $1 / 3<111>$ dislocation loops.

After annealing at $550^{\circ} \mathrm{C}$ or above, the anorphous layer was recrystallized and two layers of dislocation loops were left at the region which had been the transition region. 13 With further annealing the 1/3 <111> type loops converted to perfect loops by nucleation of a Shockley partial 1/6 e112s:

$$
\frac{1}{3}[111]+\frac{1}{6}[11 \overline{2}] \rightarrow \frac{1}{2}[110]
$$


Nucleation of this Shockley partial dislocation is possibly aided by internal stresses during annealing for recrystallization ${ }^{122}$ or shear stresses produced by the different density of amorphous zones in the crystalline matrix.

Higher temperature $\left(900^{\circ} \mathrm{C}\right)$ for longer time $(-30 \mathrm{~min})$ can drive these perfect dislocation loops to climb toward the surface. 123 However, if the density of extrinsic stacking faults is high enough after implantation, then the annealing of this specimen would not only convert faulted loops to perfect loops, but also the perfect loops will coarsen and meet each other and interact to form the dislocation networks, which are usually observed in the post-annealed specimens of nigh dose $\left(1 \times 10^{15} \sim 3 \times 10^{16} / \mathrm{cm}^{2}\right)$ boron ion implantation at room temperature. This kind of network is yery difficult to anneal out even at very high temperature. ${ }^{12}$ The oniy method to solve this problem is to produce continuous amorphous layers in the first place by low temperature or high beam current boron implantation. A preamorphized layer by heavy ions such as Ge, As, or even Si, can also serve as a starting material for further boron implantation. 


\section{SURARY}

The principal works of this study are sumarized below.

1. Amorphization during light ion damage is proposed to consist of two stages: first stage, point defects or slightly larger clusters and complexes build up to a critical concentration; second stage, amorphous zones are formed near the end of an ion track when it terminates within a volume having more than the critical concentration of point defects and/or clusters.

2. Depenaing on the uniformity of point defect distribution two possibilities for the second stage of amorphization are suggested for the ion implantation at different temperatures and different ion masses:

a) At low temperature where vacancies are immobile, the buildup of point defects is uniform throughout the irradiated volume and continuous amorphous layers results from the eventual overlap of amorphous zones formed with random distribution.

b) At room temperature migration of point defects results in larger average size of the point defect clusters or complexes, and in their nonuniform distribution with in the irradiated volume. There is a less clear separation betwern stage 1 and stage 2, because at given fluence the critical point defect density may exist in only a fraction of the irradiated volume. Then some amorphous ragions are forned, their strain field ay increase the nonuniformity of point defect clustering so that new amorphous material is preferentially formed near already existing amorphous zones. 
3. The amorphization mechanism for boron ion implantation varies with temper_cure: (1) The over lap model (Gibbons) at temperature near $0^{\circ} \mathrm{K}$, (2) mechanism $A$ at liquid nitrogen temperature, (3) mechanism B at room temperature.

4. Pairs of dinterstitials and divacancies which are the smallest stable point defect clusters for room temperature implantation, are considered as embryos of amorphous zones. The bonding structures around these di interstitial-divacancy pairs can be rebonded into five to eight fold rings which is characteristic of the amorphous structure by an additional light ion bombardment.

5. The presence of both vacancy type and interstitial type point defect clusters for amorphization is consistent with the small density difference $(1-2 \%)$ for amorphous silicon compared to crystalline material. The mobility of interstitials at liquid nitrogen temperature and their consequent diffusion to the surface provides an explanation for the observed increases of the critical deposition energy for amorphization in low energy implantation only if it is assumed that interstitials do play an essential role in the amorphization mechanism.

6. The ratio of the critical deposition energy for $100 \mathrm{keV}$ boron impiantation to that of $4 \mathrm{MeV}$ implantation is about 7.3, which is close to the relative loss rate of interstitials due to outdiffusion to surface for the two cases $(-6.7)$.

7. The formation of small amorphous zones by boron ion implantation at room temperature was observed by cross-section high resolution 
TEM lattice images and confirmed by Electron Paramagnetic Resonance spectra.

8. For boron, a continuous amorphization layer cannot be formed at roorn temperature unless a high bean current is employed, because a large fraction of the interstitial atoms segregate into extrinsic stacking fault loops and the interstitials or dinterstitials required for amorphization are no longer available in the surrounding crystalline material.

9. At room temperature where point defects and implanted boron atoms are mobile, (113), 1/3 (111) extrinsic and other smaller distortion $1 / x$ (111) stacking faults are formed in the still crystalline material during the amorphization process. These stacking faults are the origin of secondary defects formed during annealing. 


\section{CONCLUSION}

Amorphization by implantation of boron ions (which is the lightest element generally used in I.C. fabrication processes) has been systematically studied for various temperatures, various voltages and various dose rates. Based on theoretical considerations and experimental results, a new amorphization model for light and intermediate mass ion damage is proposed consisting of two-stages. The role of interstitial type point defects or clusters in amorphization is emphasized. Due to the higher mobility of interstitials out-diffusion to the surface particularly during amorphization with low energy can be significant. From a review of the idealized amorphous structure, diinterstitialdivacancy pairs are suggested to be the embryos of amorphous zones formed auring room temperature implantation. The stacking fault loops found in specimens implanted with boron at room temperature are considered to be the origin of secondary defects formed during annealing. 


\section{ACKHOMLEDGEENTS}

This work would not have been possible without the support, encouragement and guidance of my research advisor, Professor Jack Washburn. His patience and understanding to a foreign student is incomparable. His bright suggestions always inspire me to solve fundamental questions. It is one of the most enjoyable things in my life to aiscuss with him.

1 would like to express my appreciation and gratitude to Professors Ron Gronsky and Eicke Weber for the ir helpful discussions on TEM and EPR theories and techniques. Many thanks also go to Professor. Shyn Wang for reading my thesis and for serving on my exam committee. Special thanks are due to Peter Byrne for preparing MeV boron ion implantation specimens, and to Dave Ackland for his technical support on high resolution TEM.

My unforgettable memories in Berkeley are due to the warm and friendly atmosphere in the Washburn and Weber research groups. Without the stimulating discussions and assistance from my friends, this work could not have proceeded as smoothly. I would like to thank my friends, Ors. Devendra Sadana, Tim Sands and Pei-Ching Ling, and Nan-Ray Wu, Jurek Mazur, and Ettien Colas. I would also like to thank the Technical Information Division at Lawrence Berkeley Laboratory for their expert assistance in preparing this manuscript, with special thanks to Connie Silva for her careful typing and editing.

Most of all, I wish to thank my wife, Chai Lan, for her consideration and support during this period. Finally, the umavering love I 
received from my parents was essential to me in order to finish this work.

This work was supported by the Division of Materials Science, Office of Basic Energy Science, U.S. Department of Energy, under Contract No. DE-AC03-76SF00098. 


\section{REFEREACES}

1. J.M. Fairfield and B.L. Crowder, Trans. of Met. SoC. of AIME, 245, $469(1969)$.

2. G. Cter and W.A. Grant, "Ion Implantation in Semiconductors," John Wiley and Sons, New York (1976).

3. G. Dearnaley, J.H. Freeman, R.S. Nelson and J. Stephen, Ion Implantation, North Holland Pub. Co. 1973.

4. S.M. Darison and G.R. Booker, Rad. Eff. V6, 33 (1970).

5. J.W. Corbett, Rad. Eff. V6, 3 (1970).

6. J.F. Gibbons, Rad. Eff. V6, 313 (1975).

7. S. Prussin, in "Ion Implantation in Semiconductors" (edited by S. Namba), Plenum, New York, p. 449 (1975).

8. J. Narayan, Proc. of the 35th Ann. Meeting, EMSA (edited by G.W. Bailey), p. 296 (1977).

9. R.M. Drosd, "A Model of the Recrystallization Mechanism of Amorphous Silicon Layers Created by Ion Implantation," Ph.D. thes is, University of California, Berkeley, November 1979.

10. D.E. Davies, Appl. Phys. Lett. 14, 227 (1969).

11. R.R. Hart and 0.J. Marsh, App1. Phys. Lett. 15, 206 (1969).

12. D.K. Sadana, S.C. Shatas and A. Gat, Microsco. Semicond. Mater. Conf. Inst. Phys. Conf. Sec. Ho. 67, 143 (1983).

13. D.K. Sadana, J. Washburn and G.R. Bookēr, Phil. Mag. B. V46, 611 (1982).

14. L.T. Chadderton and F.H. Eisen, Rad. Eff. Y7, 129 (1971). 
15. S.M. Cheruvi, "Crystalline to Amorphous Transformation in Silicon," Ph.D. thesis, University of Cal ifornia, Berkeley, September 1982 .

16. S. Pruss in, D.I. Margolese, and R.N. Tauber, J. Appl. Phys. 54, 2316 (1983).

17. P. Byrne, J. Appl. Phys. 54 (2), 1146 (1983).

18. L.T. Chadderton, "Radiation Damage in Crystals," Methaen, London, 1965 .

19. J. Lindhard and M. Scharff, Phys. Rev. 124, 128 (1961).

20. J. Lindhard, M. Scharff, and H.E. Schiott, Kgl. Danske Vidensab Seiskab. Mat. Phys. Medd. 33, No. 14 (1963).

21. J.W. Mayer, L. Eriksson, and J.A. Davies, "Ion Implantation in Semiconductor," Chapter 2, Academic Press, New York and London, 1970.

22. W.S. Johnson and J.F. Gibbons, "Projected Range Statistics in Semiconductors," Stanford University Bookstore, 1970.

23. G.H. Kinch in and R.S. Pease, "The displacement of atoms in solids by radiation," Rep. Progr. Phys. V18, 2 (1955).

24. L.A. Christel, J.F. Gibbons and T.W. Sigmon, J. Appl. Phys. 52, $7143(1981)$.

25. J. Washburn, C.S. Murty, D.K. Sadana, P, Byrne, R. Gronsky, N. Cheung, and R. Killaas, Nuclear Inst, and Meth. 209, 345 (1983).

26. F.F. Morehead, Jr., and B.L. Crowder, Rad. Eff. Y6, 27 (1970). 27. J.F. Gibtons, "Lectures on Ion Implentation and Proton Enhanced Diffusion," University of Tokyo, Tokyo, Japan; Jap. Soc. Appl. Phys., August 1977. 
28. E.C. Baranova, Y.M. Gusev, Yu. Y. Martynenko, C.Y. Starin in and 1.B. Haibull in; Rad. Eff. 18, 21 (1973).

29. "Fundamental Physics of Amorphous Semiconductors," Proceeding of the Kyoto Summer Institute, Kyoto, Japan, September 8-11, 1980; edited by F. Yonozawa, Springer Series in Solid-State Sciences V25, New York (1980).

30. D.E. Polk, J. Non-Cryst. Solids $\underline{5}, 365$ (1971).

31. W.H. Zachariasen, J. Amer. Chem. Soc. 54, 3841 (1932).

32. D.E. Poik and D.S. Bondreaux, Phys. Rev. Lett. $\underline{31}, 92$ (1973).

33. Spaepen, Acta Metall. V26, 1167 (1978).

34. S.C. Moss, Proc, 5th Int. Conf. "Amorphous and Liquid Semiconductors," Gearmisch, edited by J. Stuke and W. Brenig, p. 17, Taylor and Francis, London (1973).

35. S.C. Moss and D. Adler, Comm. Solid St. Phys. $\underline{5}, 47$ (1973).

36. W. Paul and G.A.N. Connell, Physics of structurally disordered solias, edited by S.S. Mitra, p. 45, Plenum Press, New York (1974).

37. D.E. Polk, J. Non-Crystall ine Sol ids $\underline{5}, 365$ (1971).

38. P. Steinhardt, R. Alben, and D. Weaire, J. Non-Crystalline Solids 15, 199 (1974).

39. G.A.H. Connell and R.J. Tenkin, Phys. Rev. (B) 9 , 5323 (1974).

40. D.S. Boudreaux, D.E. Polk and M.G. Eu. iyy, Proc. Int. Conf. Tetrahedrally Bonded Amorphous Semiconductors, A.I.P. Conf. Proc. Y20, 206 (1974).

41. M.J. Shevchick and M. Pewl, J. Won-Crystall ine Sol ids 8-10, 381 (1972). 
42. J.C. Phillips, J. Non-Cryst. Solids, 34, 153 (1970).

43. D.K. Biegelsen, ProC. of 1982 MRS Symposium, "Defects in Semiconductors," Boston, Nov. 1982.

44. M.H. Brodsky and R.S. Title, Phys. Rev. Let. $\underline{23}, 581$ (1969).

45. S. Veprek, Z. Iqbal and F.-A. Sarott, Phil. Mag. B45, 137 (1982).

46. J. Pauling, "The Nature of the Chemical Bond," Cornell University Press, New York (1960).

47. J. Robertson, Phy. Chem. Glasses 23, 1 (1982).

48. M.H. Brodsky, R.S. Title, K. Weiser and G.D. Pettit, Phys. Rev. B1, 2632 (1970).

49. B.L. Crowder, R.S. Title, M.H. Brodsky, and G.D. Pettit, App T. Phys. Lett. 16, 205 (1970).

50. K.L. Brower and W. Beezhold, J. Appl. Phys. 43, 3499 (1972).

51. S. Veprek, Z. Iqbal, H.R. Oswald, F.-A. Sarott, J.J. Wagner, and A.P. Webb, Solid St. Commun. 39, 509 (1981).

52. H.P. Klug and L.E. Alexander, "X-ray Diffraction Procedures," Wiley, New York (1974).

53. C. Kittel, "Introduction to Sol id State Physics," 4 th edition, Wiley, New York (1971).

54. M.L. Rudee, and A. Howie, Phil. Mag. 25, 1001 (1972).

55. R. Dandoloff, G.H. Dohler, and H. Bilz, J. Non-crystalline Solid, $35-36,537$ (1980).

56. G.H. Dohler, R. Dandoloff, and H. Bilz, J. Mon-crystall ine Sol id, 42. 87 (1980).

57. F. Spaepen, Phil. Mag. 30, 417 (1974). 
58. J. Marayan, D. Fathy, O.S. Den, and O.W. Holl and, J. Vac. Sci. Technol. A2(3), 1303 (1984).

59. G. Muller and S. Kalbitzer, Proc. of the 4 th Inter. Conf. on Non-crystalline Solids, edited by G.E. Frischat, Trans. Tech. 278 (1977).

60. 2. Iqbal and S. Vepi 3k, J. Phys. C15 377 (1982).

61. T. Kamiya, M. Kishi, A. Ushirokawa and T. Katoda, App1. Phys. Lett. $\underline{38}, 377$ (1981).

62. W.E. Spear, G. WillekE, D.G. LeComber and A.G. Fitzgerald, Physica B and C 117-118, 908 (1983).

63. M.H. Brodsky, "Light scattering in solids," edited by M. Cardona, Springer-Verlag, Berlin (1975).

6i. H. Fritzsche, Proc. of the Kyoto Summer Institute, Sept. 1980, "Fundamental Physics of Amorphous Semiconductors," p.1, edited by F. Yonezawa, Springer Verlag, New York (1981).

65. J.C. Phillips, J. Non-Cryst. Solids, 34, 153 (1979).

66. J.W. Mayer, J.A. Davies and L. Erikssol. "Ion Implantation in Si and Ge," Chapter 2. Academic Press, New York (1970).

67. F.F. Morehead, B.L. Crowder and R.S. Title, J. Appl. Phy's. V43, 1112 (1972).

68. S. Chou, "Investigation of impurities in silicon by ion scattering," Ph.D. dissertation, Stanford Univ., Stanford, CA, June $19 ? 1$.

69. H. Frank, "Lattice Defects in Semiconductors," Institute of Physics, London, edited by F.A. Huntley, p, 40 (1974). 
70. J.F. Gibbons, Proc. of the IEEE, Y60, 1062 (1972).

71. J.R. Dennis and E. Hale, J. Appl. Phys. 49, 1119 (1978).

72. R. Hebb and G. Carter, Rad. Eff. Y42, 159 (1979).

73. G. Carter and R. Webb, Rad. Eff. Lett. V43, 19 (1979).

74. R. Webb and G. Carter, Rad. Eff. V59, 69 (1981).

75. F.L. Vook and H.J. Stein, Rad. Eff. V2, 23 (1969).

76. L.J. Cheng, J.C. Corelli, J.W. Corbett, and G.D. Watk ins, Phys. Rev. 152, 761 (1966).

77. F.L. Vook and H.J. Stein, Rad. Eff. V6, 11 (1970).

78. H.J. Stein, F.L. Vook, D.K. Brice, J.A. Borders, and S.T. Picraux, Rad. Eff. V6, 19 (1970).

79. M.L. Swanson, J.T. Parsons and C.W. Hoelke, Rad. Eff. yg, 249 (1971).

80. L.T. Chadderton, Rad. Eff. V8, 77 (1971).

81. H.J. Stein, F.L. Vook, D.K. Brice, J.A. Borders, and S.T. Picraux, Rad. Eff. V6, 19 (1970).

82. D.K. Brice, Rad. Eff. 트, 77 (1970).

83. J.F. Gibbons, Proc. U.S.-Japan Sci. Exchange on Ion Implantation, Kyoto, Japan, S. Namba, Ed. Japan Soc. for the Promotion of Sci., p. 39-93 (1972).

84. L.C. Feldman and J.W. Rodgers, J. Appl. Phys. V41, 3776 (1970).

85. D.A. Masden ana J.L. Whitton, Rad. Eff. Y6, 181 (1970).

8c. E. Bpgh, P. Hogild and I. Stensgaard, Rad. Eff. V7, 115 (1971).

87. F. Ohira and M. Itakura, Jap. J. Appl. Phys. V21, 42 (1982).

88. P.F. Byrne, N.H. Cheung and D.K. Sadana, Appl. Phys. Lett. v4l, 537 (1982). 
89. P.F. Byrne, M.H. Cheung and D.K. Sadana; private commication (1983).

90. Y. Shih, T. Washburn, E.R. Weber and R. Gronsky. Proc. of 1985 MRS spring meeting, San Francisco, "Ion Beam Process in Advanced Electronic Materials and Device Technology," edited by F.H. Eisen, T.W. Sigmon, and B.R. Appleton, p. 65 (1985).

91. E.P. EerNisse, App 1. Phys. Lett. v18, 581 (1971).

92. G.S. Oehrlein, I. Krafcsik, J.L. Lindstrom, A.E. Jaworowski, and J.W. Corbett, J. App 1. Phys. V54, 179 (1983).

93. A.R. Bean, R.C. Newman, and R.S. Smith, J. Phys. Chem. Solids V31, $739(1970)$.

94. A.R. Bean, S.R. Morrison, R.C. Newman, and R.S. Smith, J. Phys. C5, 379 (1972).

95. J.W. Corbett, J.C. Bourgoin, L.J. Cheng, J.C. Corelli, Y.H. Lee, P.M. Mooney and C. Weiget, in "Radiation Effects in Semiconductor 1976" edited by N.B. Ur Ii and J.W. Corbett (Inst. Phys. Bristol-London, 1977) p. 1.

96. J.W. Corbett, J.P. Karins and T.Y. Tan, Nuclear Instrum. and Meth. v182, 457 (1981).

97. W. Jung and G.S. Newell, Phys. Rev. V132, 648 (1963).

98. Y.H. Lee, N.N. Gerasimenko, and J.H. Corbett, Phys. Rev. B14, 450 (1976).

99. J.H. Corbett and J.C. Bourgoin, in "Point Defects in Solids, Yol. 2," EJ's, J.H. Crawford, Jr., and L.M. Slifk in, P. 2 (PIenun, New York, 1975). 
100. 6.D. Watkins and J.H. Corbett, Phys. Rev. $\underline{138}, 1543$ (1965).

101. J.H. Corbett and G.D. Watkins, editors, "Radiation Effects in Semiconductors," Gordon and Breach, London, New York and Paris (1971).

102. A. Seegers and W. Frank, in MProc. of Intern. Conf. on Defects in Semiconductors," Reading, England, p. 262 (1972).

103. W. Beezhold and K.L. Brower, IEEE Trans. Nucl. Sci., NS20, 209 (1973).

104. N.A. Sobolev, G. Gotz, W. Karthe, and S. Schnabel, Rad. Eff. V42, $23(1979)$.

105. J.F. Ziegler, B.J. Crowder and W.J. Kleinfelder, IBM J. Res. Develop. 452 (1971).

106. D.P. Lecrosinier and G.P. Pelous, IEEE Trans. Electron Devices ED21, 113 (1974).

107. M. Doken, T. Unagami, K. Sakuma and K. Kajiyama, in: Proc. of Int. Electron Device Meet., 1981 IEEE, New York, 586 (1981).

108. P.F. Byrne, N.W. Cheung, and D.K. Sadana, Appl. Phys. Lett. V니, 537 (1982).

109. J. Fletcher, J.M. Titchmarsh and G.R. Booker, Inst. Phys. Conf. Series (London) V드, 153 (1980).

110. G. Thomas and M. Goringe, "Transmission Electron Microscopy," John Wiley and Sons, New York, p. 315 (1979).

111. 0.J. Scherzer, Appl. Phys. Y20, 20 (1949).

112. K.L. Brower, in "Wuclear and Electron Resonance Spectroscopies Applied to Materials Science," MRS Symposia Proc. Vol. 3 ledited 
by E.N. Kaufwann and G.K. Shenoy), Elsevier Morth Holland, Inc., p. 71 (1981).

113. B. Bleaney and K.H.H. Stevens, Rept. Prog. Phys. 18, 108 (1953).

114. G.D. Watkins, Phys. Rev. B12, 5824 (1975).

115. D.K. Biegelsen, in Muclear and Electron Resonance Spectroscopies Applied to Materials Science," MRS Symposia Proc. Vol. 3 (edited by E.N. Kaufman and G.K. Shenoy), Elservier North Holland, Inc., p. 85 (1981).

116. C.P. Poole, Jr., "Electron Spin Resonance, A Comprehensive Treaties on Experimental Techniques," John Wiley and Sons (1982). 117. H.F. Wolf, "Semiconductors," John Wiley and Sons, Inc., New York, p. 157 (1971).

118. M. Kiritani, in "Progress in the Study of Poini Defects," (edited by M. Doyama and S. Yoshida) University of Tokyo press, p. 247 (1977).

119. J.C. Bourgoin and J.W. Corbett, Rad. Eff. V36, 157 (1978).

120. T. Sands, D.K. Sadana, R. Gronsky, and J. Washburn, Appl. Phys. Lett. V44, 874 (1984).

121. D.K. Sadana and T. Sands, private communication.

122. K. Sheshan and E.P. EerNisse, Appl. Phys. Lett. V33, 21 (1978).

123. N.R. Wu, P. Ling, D.K. Sadana, J. Washburn, and M.I. Current, Proc. of 1983 ECS reeting, San Francisco. 


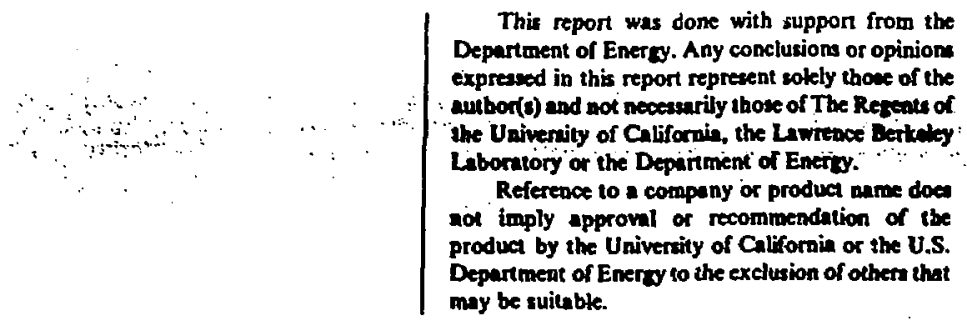

